\title{
AIBN-Induced Remote Trifluoromethyl-Alkynylation of Thioalkynes
}

Zhimin Xiong, ${ }^{\dagger}$ Fang Zhang, ${ }^{\dagger}$ Yongqi Yu, ${ }^{\ddagger}$ Ze Tan, ${ }^{\star}$ and Gangguo Zhu ${ }^{* \dagger}$

${ }^{\dagger}$ Key Laboratory of the Ministry of Education for Advanced Catalysis Materials, Department of Chemistry, Zhejiang Normal University, 688 Yingbin Road, Jinhua 321004, P. R. China. gangguo@zjnu.cn

${ }^{*}$ State Key Laboratory of Chemo/Biosensing and Chemometrics, College of Chemistry and Chemical Engineering, Hunan University, Changsha 410082, P. R. China

\section{Table of Contents}

General... 
General. Unless otherwise noted, materials obtained from commercial suppliers were used directly without further purification. ${ }^{1} \mathrm{H},{ }^{13} \mathrm{C}$, and ${ }^{19} \mathrm{~F}$ NMR spectra were measured on a $600 \mathrm{MHz}$ or $400 \mathrm{MHz}$ NMR spectrometer using $\mathrm{CDCl}_{3}$ as the solvent with tetramethylsilane (TMS) as the internal standard. The proton-decoupled ${ }^{19} \mathrm{~F}$ NMR spectroscopy was used to determine the ${ }^{19} \mathrm{~F}$ NMR data. Chemical shifts $(\delta)$ are given in parts per million relative to TMS, and the coupling constants are given in hertz. High-resolution mass spectrometry (HRMS) analysis were carried out using a TOF MS instrument with an APCI or ESI source. Column chromatography was performed using silica gel (200-300 mesh). Thioalkynes $\mathbf{1}^{1}$ and alkynyl triflones $\mathbf{2}^{2}$ were prepared according to the methods reported in the literature.

General Procedure for the AIBN-Induced Remote Trifluoromethyl-Alkynylation of Thioalkynes

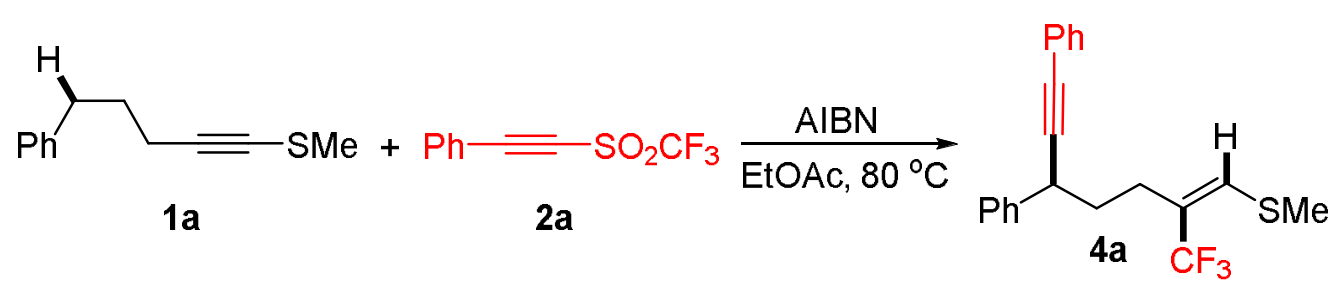

To a Schlenk flask containing 1a $(38.0 \mathrm{mg}, 0.2 \mathrm{mmol})$ and $\mathbf{2 a}(93.6 \mathrm{mg}, 0.4 \mathrm{mmol})$ in $1 \mathrm{~mL}$ of EtOAc was added AIBN (9.9 mg, $0.06 \mathrm{mmol})$ under a nitrogen atmosphere. The flask was immersed in an oil bath and heated at $80{ }^{\circ} \mathrm{C}$ for $12 \mathrm{~h}$. Then, the reaction mixture was quenched with water, extracted with EtOAc, washed with brine, dried over anhydrous $\mathrm{Na}_{2} \mathrm{SO}_{4}$, and concentrated. Column chromatography on silica gel (EtOAc/petroleum ethers $=1: 100)$ gave 55 $\mathrm{mg}(76 \%$ yield $)$ of $\mathbf{4 a}$ as a yellow oil. ${ }^{1} \mathrm{H}$ NMR $\left(600 \mathrm{MHz}, \mathrm{CDCl}_{3}\right) \delta 7.46-7.40(\mathrm{~m}, 4 \mathrm{H}), 7.35(\mathrm{t}, J$ $=7.6 \mathrm{~Hz}, 2 \mathrm{H}), 7.33-7.29(\mathrm{~m}, 3 \mathrm{H}), 7.27$ (d, $J=7.3 \mathrm{~Hz}, 1 \mathrm{H}), 6.34(\mathrm{~s}, 1 \mathrm{H}), 3.87$ (dd, $J=9.1,5.6 \mathrm{~Hz}$, 1H), 2.49-2.41 (m, 2H), $2.32(\mathrm{~s}, 3 \mathrm{H}), 2.04-1.93(\mathrm{~m}, 2 \mathrm{H}),{ }^{13} \mathrm{C} \mathrm{NMR}\left(151 \mathrm{MHz}, \mathrm{CDCl}_{3}\right) \delta 141.3$, $136.1(\mathrm{q}, J=3.2 \mathrm{~Hz}), 131.6,128.6,128.3,128.0,127.4,127.0,124.5$ (q, $J=275.2 \mathrm{~Hz}), 123.4$, $123.2(\mathrm{q}, J=29.7 \mathrm{~Hz}), 90.4,84.2,37.6,37.3,30.6$ (q, $J=1.6 \mathrm{~Hz}), 18.5(\mathrm{q}, J=2.1 \mathrm{~Hz}) ;{ }^{19} \mathrm{~F}$ NMR

\footnotetext{
${ }^{1}$ (a) Yang, Z.; Chen, X.; Kong, W.; Xia, S.; Zheng, R.; Luo, F.; Zhu, G. Org. Biomol. Chem. 2013, 11, 2175. (b) Zhu, G.; Kong, W.; Feng, H.; Qian, Z. J. Org. Chem. 2014, 79, 1786.

${ }^{2}$ Xiang, J. S.; Mahadevan, A.; Fuchs, P. L. J. Am. Chem. Soc. 1996, 118, 4284.
} 
(565 MHz, $\left.\mathrm{CDCl}_{3}\right) \delta-61.7$; HRMS (APCI-TOF) calcd for $\mathrm{C}_{21} \mathrm{H}_{20} \mathrm{~F}_{3} \mathrm{~S}(\mathrm{M}+\mathrm{H})^{+} 361.1232$, found 361.1226 .

\section{Scale-Up Synthesis of 4a.}

To a Schlenk flask containing 1a (380 mg, $2.0 \mathrm{mmol})$ and 2a (936 mg, $4.0 \mathrm{mmol})$ in $10 \mathrm{~mL}$ of EtOAc was added AIBN (99 mg, $0.6 \mathrm{mmol}$ ) under a nitrogen atmosphere. The flask was immersed in an oil bath and heated at $80{ }^{\circ} \mathrm{C}$ for $12 \mathrm{~h}$. Then, the reaction mixture was quenched with water, extracted with EtOAc, washed with brine, dried over anhydrous $\mathrm{Na}_{2} \mathrm{SO}_{4}$, and concentrated. Column chromatography on silica gel (EtOAc/petroleum ethers $=1: 100)$ gave 568 mg $(79 \%$ yield $)$ of $\mathbf{4 a}$ as a yellow oil.<smiles>COc1ccccc1C(C)(F)F</smiles>

Compound $4 \boldsymbol{b}$ : $58 \mathrm{mg}, 77 \%$ yield, yellow oil; Flash column chromatography conditions: EtOAc/petroleum ethers $=1: 100 ;{ }^{1} \mathrm{H}$ NMR $\left(600 \mathrm{MHz}, \mathrm{CDCl}_{3}\right) \delta 7.44(\mathrm{dd}, J=6.2,2.7 \mathrm{~Hz}, 2 \mathrm{H})$, $7.32-7.29(\mathrm{~m}, 5 \mathrm{H}), 7.16(\mathrm{~d}, J=7.8 \mathrm{~Hz}, 2 \mathrm{H}), 6.33(\mathrm{~s}, 1 \mathrm{H}), 3.83(\mathrm{dd}, J=8.9,5.7 \mathrm{~Hz}, 1 \mathrm{H})$, $2.48-2.40(\mathrm{~m}, 2 \mathrm{H}), 2.34(\mathrm{~s}, 3 \mathrm{H}), 2.32(\mathrm{~s}, 3 \mathrm{H}), 2.00-1.93(\mathrm{~m}, 2 \mathrm{H}) ;{ }^{13} \mathrm{C} \mathrm{NMR}\left(151 \mathrm{MHz}, \mathrm{CDCl}_{3}\right) \delta$ $138.3,136.6,136.0(\mathrm{q}, J=3.1 \mathrm{~Hz}), 131.6,129.3,128.3,127.9,127.3,124.5(\mathrm{q}, J=275.3 \mathrm{~Hz})$, $123.5,123.3$ (q, $J=29.6 \mathrm{~Hz}), 90.7,84.0,37.3,37.2,30.6$ (q, $J=1.6 \mathrm{~Hz}), 21.0,18.5$ (q, $J=2.1 \mathrm{~Hz})$; ${ }^{19} \mathrm{~F}$ NMR (565 MHz, $\left.\mathrm{CDCl}_{3}\right) \delta-61.7$; HRMS (APCI-TOF) calcd for $\mathrm{C}_{22} \mathrm{H}_{22} \mathrm{~F}_{3} \mathrm{~S}(\mathrm{M}+\mathrm{H})^{+} 375.1389$, found 375.1384 .<smiles>CC(=CCC(C#Cc1ccccc1)c1cccc(C)c1)C(F)(F)F</smiles>

Compound $4 c$ : $55 \mathrm{mg}, 74 \%$ yield, yellow oil; Flash column chromatography conditions: EtOAc/petroleum ethers $=1: 100 ;{ }^{1} \mathrm{H}$ NMR $\left(600 \mathrm{MHz}, \mathrm{CDCl}_{3}\right) \delta 7.52-7.47(\mathrm{~m}, 2 \mathrm{H}), 7.35(\mathrm{dd}, J=$ 4.9, 1.4 Hz, 3H), 7.28-7.26 (m, 3H), $7.12(\mathrm{~d}, J=7.0 \mathrm{~Hz}, 1 \mathrm{H}), 6.38$ (s, 1H), 3.87 (dd, $J=9.0,5.7$ $\mathrm{Hz}, 1 \mathrm{H}), 2.54-2.47$ (m, 2H), 2.41 (s, 3H), $2.36(\mathrm{~s}, 3 \mathrm{H}), 2.07-1.96(\mathrm{~m}, 2 \mathrm{H}) ;{ }^{13} \mathrm{C}$ NMR $(151 \mathrm{MHz}$, 
$\left.\mathrm{CDCl}_{3}\right) \delta 141.2,138.3,136.1(\mathrm{q}, J=3.1 \mathrm{~Hz}), 131.6,128.5,128.3,128.1,127.9,127.7,124.5(\mathrm{q}, J$ $=275.2 \mathrm{~Hz}), 124.4,123.3(\mathrm{q}, J=29.6 \mathrm{~Hz}), 123.5,90.6,84.0,37.5,37.3,30.6(\mathrm{q}, J=1.7 \mathrm{~Hz}), 21.5$, $18.5(\mathrm{q}, J=2.1 \mathrm{~Hz}) ;{ }^{19} \mathrm{~F}$ NMR $\left(565 \mathrm{MHz}, \mathrm{CDCl}_{3}\right) \delta-61.7$; HRMS (APCI-TOF) calcd for $\mathrm{C}_{22} \mathrm{H}_{22} \mathrm{~F}_{3} \mathrm{~S}(\mathrm{M}+\mathrm{H})^{+} 375.1389$, found 375.1383 .<smiles>C/C=C(\CCC(C#CPc1ccccc1)c1ccccc1C)C(F)(F)F</smiles>

4d

Compound 4d: $45 \mathrm{mg}, 60 \%$ yield, yellow oil; Flash column chromatography conditions: EtOAc/petroleum ethers $=1: 100 ;{ }^{1} \mathrm{H}$ NMR $\left(600 \mathrm{MHz}, \mathrm{CDCl}_{3}\right) \delta 7.57(\mathrm{~d}, J=7.6 \mathrm{~Hz}, 1 \mathrm{H}), 7.44(\mathrm{dd}$, $J=6.3,2.7 \mathrm{~Hz}, 2 \mathrm{H}), 7.32-7.27(\mathrm{~m}, 3 \mathrm{H}), 7.22(\mathrm{dd}, J=7.8,5.1 \mathrm{~Hz}, 1 \mathrm{H}), 7.16(\mathrm{~d}, J=4.0 \mathrm{~Hz}, 2 \mathrm{H})$, $6.36(\mathrm{~s}, 1 \mathrm{H}), 4.04(\mathrm{dd}, J=9.7,5.0 \mathrm{~Hz}, 1 \mathrm{H}), 2.57-2.44(\mathrm{~m}, 2 \mathrm{H}), 2.37$ (s, 3H), 2.31 (s, 3H), 1.97-1.85 (m, 2H); ${ }^{13} \mathrm{C}$ NMR (151 MHz, $\left.\mathrm{CDCl}_{3}\right) \delta 139.5,136.2(\mathrm{q}, J=3.2 \mathrm{~Hz}), 134.8,131.6$, 130.6, 128.3, 127.9, 127.5, 126.9, 126.4, 124.5 (q, $J=275.2 \mathrm{~Hz}), 123.5,123.1$ (q, $J=29.8 \mathrm{~Hz})$, 90.8, 83.6, 35.6, 34.1, 30.8 (q, $J=1.6 \mathrm{~Hz}), 19.1,18.5$ (q, $J=2.1 \mathrm{~Hz}) ;{ }^{19} \mathrm{~F}$ NMR $\left(565 \mathrm{MHz}, \mathrm{CDCl}_{3}\right)$ $\delta$-61.7; HRMS (APCI-TOF) calcd for $\mathrm{C}_{22} \mathrm{H}_{22} \mathrm{~F}_{3} \mathrm{~S}(\mathrm{M}+\mathrm{H})^{+} 375.1389$, found 375.1384.<smiles>COc1ccc(C(C#Cc2ccccc2)CCC(C)=CC(C)(F)F)cc1</smiles>

Compound $4 \boldsymbol{e}$ : $65 \mathrm{mg}, 83 \%$ yield, yellow oil; Flash column chromatography conditions: EtOAc/petroleum ethers $=1: 100 ;{ }^{1} \mathrm{H}$ NMR $\left(600 \mathrm{MHz}, \mathrm{CDCl}_{3}\right) \delta 7.44(\mathrm{dd}, J=6.5,3.0 \mathrm{~Hz}, 2 \mathrm{H})$, 7.34-7.29 (m, 5H), 6.89 (d, $J=8.6 \mathrm{~Hz}, 2 \mathrm{H}), 6.33(\mathrm{~s}, 1 \mathrm{H}), 3.84-3.79(\mathrm{~m}, 4 \mathrm{H}), 2.47-2.41(\mathrm{~m}, 2 \mathrm{H})$, $2.32(\mathrm{~s}, 3 \mathrm{H}), 2.00-1.91(\mathrm{~m}, 2 \mathrm{H}) ;{ }^{13} \mathrm{C}$ NMR $\left(151 \mathrm{MHz}, \mathrm{CDCl}_{3}\right) \delta 158.5,136.0$ (q, $\left.J=3.1 \mathrm{~Hz}\right)$, 133.4, 131.6, 128.4, 128.3, 127.9, $124.5(\mathrm{q}, J=275.3 \mathrm{~Hz}) 123.5,123.3(\mathrm{q}, J=29.6 \mathrm{~Hz}), 90.8,84.0$, $55.3,37.4,36.7,30.5$ (q, $J=1.7 \mathrm{~Hz}), 18.5(\mathrm{q}, J=2.1 \mathrm{~Hz}) ;{ }^{19} \mathrm{~F}$ NMR $\left(565 \mathrm{MHz}, \mathrm{CDCl}_{3}\right) \delta-61.7$; HRMS (APCI-TOF) calcd for $\mathrm{C}_{22} \mathrm{H}_{22} \mathrm{~F}_{3} \mathrm{OS}(\mathrm{M}+\mathrm{H})^{+} 391.1338$, found 391.1337 . 
<smiles>CC(=CCCC(C#Cc1ccccc1)c1ccc2c(c1)OCO2)C(F)(F)F</smiles>

Compound $4 f$ : $47 \mathrm{mg}, 58 \%$ yield, yellow oil; Flash column chromatography conditions: EtOAc/petroleum ethers $=1: 50 ;{ }^{1} \mathrm{H}$ NMR $\left(600 \mathrm{MHz}, \mathrm{CDCl}_{3}\right) \delta 7.44(\mathrm{dd}, J=6.3,2.7 \mathrm{~Hz}, 2 \mathrm{H})$, 7.32-7.29 (m, 3H), $6.93(\mathrm{~s}, 1 \mathrm{H}), 6.85(\mathrm{dd}, J=8.0,1.1 \mathrm{~Hz}, 1 \mathrm{H}), 6.77$ (d, $J=8.0 \mathrm{~Hz}, 1 \mathrm{H}), 6.33(\mathrm{~s}$, $1 \mathrm{H}), 5.95(\mathrm{~s}, 2 \mathrm{H}), 3.79$ (dd, $J=8.6,6.0 \mathrm{~Hz}, 1 \mathrm{H}), 2.48-2.39(\mathrm{~m}, 2 \mathrm{H}), 2.33(\mathrm{~s}, 3 \mathrm{H}), 1.98-1.90(\mathrm{~m}$, $2 \mathrm{H}) ;{ }^{13} \mathrm{C}$ NMR $\left(151 \mathrm{MHz}, \mathrm{CDCl}_{3}\right) \delta 147.8,146.5,136.1$ (q, $\left.J=3.2 \mathrm{~Hz}\right), 135.2,131.6,128.3$, $128.0,124.5(\mathrm{q}, J=274.1 \mathrm{~Hz}), 123.4,123.2(\mathrm{q}, J=29.8 \mathrm{~Hz}), 120.5,108.2,107.9,101.0,90.5$, 84.1, 37.4, 37.3, 30.5 (q, $J=1.7 \mathrm{~Hz}), 18.5(\mathrm{q}, J=2.1 \mathrm{~Hz}) ;{ }^{19} \mathrm{~F}$ NMR $\left(565 \mathrm{MHz}, \mathrm{CDCl}_{3}\right) \delta-61.7$; HRMS (APCI-TOF) calcd for $\mathrm{C}_{22} \mathrm{H}_{20} \mathrm{~F}_{3} \mathrm{O}_{2} \mathrm{~S}(\mathrm{M}+\mathrm{H})^{+}$405.1131, found 405.1127 .<smiles>COc1ccc(C(C#Cc2ccccc2)CCC(C)=CC(C)(F)F)cc1</smiles>

Compound 4g: $59 \mathrm{mg}, 73 \%$ yield, yellow oil; Flash column chromatography conditions: EtOAc/petroleum ethers $=1: 100 ;{ }^{1} \mathrm{H}$ NMR $\left(600 \mathrm{MHz}, \mathrm{CDCl}_{3}\right) \delta 7.44(\mathrm{dd}, J=6.6,3.0 \mathrm{~Hz}, 2 \mathrm{H})$, $7.34(\mathrm{~d}, J=8.3 \mathrm{~Hz}, 2 \mathrm{H}), 7.32-7.29(\mathrm{~m}, 3 \mathrm{H}), 7.25(\mathrm{~d}, J=8.2 \mathrm{~Hz}, 2 \mathrm{H}), 6.33(\mathrm{~s}, 1 \mathrm{H}), 3.83(\mathrm{dd}, J=$ 8.9, $5.7 \mathrm{~Hz}, 1 \mathrm{H}), 2.48(\mathrm{~s}, 3 \mathrm{H}), 2.47-2.39(\mathrm{~m}, 2 \mathrm{H}), 2.32(\mathrm{~s}, 3 \mathrm{H}), 2.02-1.88(\mathrm{~m}, 2 \mathrm{H}) ;{ }^{13} \mathrm{C}$ NMR $(151$ $\left.\mathrm{MHz}, \mathrm{CDCl}_{3}\right) \delta 138.3,136.9,136.2(\mathrm{q}, J=3.1 \mathrm{~Hz}), 131.6,128.3,128.0,127.9,127.0,124.5(\mathrm{q}, J$ $=275.2 \mathrm{~Hz}), 123.4,123.2(\mathrm{q}, J=29.7 \mathrm{~Hz}), 90.2,84.2,37.2,37.1,30.5$ (q, $J=1.6 \mathrm{~Hz}), 18.5(\mathrm{q}, J=$ $2.1 \mathrm{~Hz}$ ), 16.0; ${ }^{19} \mathrm{~F}$ NMR (565 MHz, $\left.\mathrm{CDCl}_{3}\right) \delta-61.7$; HRMS (APCI-TOF) calcd for $\mathrm{C}_{22} \mathrm{H}_{22} \mathrm{~F}_{3} \mathrm{~S}_{2}$ (M $+\mathrm{H})^{+}$407.1110, found 407.1096.<smiles>CO/C=C(\CCC(C#CC#Cc1ccccc1)c1ccc(-c2ccccc2)cc1)C(F)(F)F</smiles>

4h 
Compound $4 \boldsymbol{h}$ : $54 \mathrm{mg}, 62 \%$ yield, yellow oil; Flash column chromatography conditions: EtOAc/petroleum ethers $=1: 100 ;{ }^{1} \mathrm{H}$ NMR $\left(600 \mathrm{MHz}, \mathrm{CDCl}_{3}\right) \delta 7.61-7.56(\mathrm{~m}, 4 \mathrm{H}), 7.50-7.44(\mathrm{~m}$, 6H), 7.35-7.30 (m, 4H), 6.36 (s, 1H), 3.92 (dd, $J=9.1,5.6 \mathrm{~Hz}, 1 \mathrm{H}), 2.49-2.39$ (m, 2H), 2.32 (s, 3H), 2.08-1.95 (m, 2H); $\left.{ }^{13} \mathrm{C} \mathrm{NMR} \mathrm{(151} \mathrm{MHz,} \mathrm{CDCl}_{3}\right) \delta 140.8,140.4,139.9,136.2$ (q, $\left.J=3.1 \mathrm{~Hz}\right)$, $131.7,131.6,128.8,128.3,128.0,127.8,127.4,127.2,127.0,124.5$ (q, $J=275.0 \mathrm{~Hz}), 123.2(\mathrm{q}, J$ $=29.7 \mathrm{~Hz}), 90.3,84.3,37.3,37.2,30.6(\mathrm{q}, J=1.6 \mathrm{~Hz}), 18.5(\mathrm{q}, J=2.1 \mathrm{~Hz}) ;{ }^{19} \mathrm{~F}$ NMR $(565 \mathrm{MHz}$, $\left.\mathrm{CDCl}_{3}\right) \delta-61.7$; HRMS (APCI-TOF) calcd for $\mathrm{C}_{27} \mathrm{H}_{24} \mathrm{~F}_{3} \mathrm{~S}(\mathrm{M}+\mathrm{H})^{+} 437.1545$, found 437.1538 .<smiles>CO/C=C(\CCC(C#Cc1ccccc1)c1ccc(F)cc1)C(F)(F)F</smiles>

Compound 4i: $48 \mathrm{mg}, 63 \%$ yield, yellow oil; Flash column chromatography conditions: EtOAc/petroleum ethers = 1:100; ${ }^{1} \mathrm{H}$ NMR $\left(600 \mathrm{MHz}, \mathrm{CDCl}_{3}\right) \delta 7.47-7.42(\mathrm{~m}, 2 \mathrm{H}), 7.39-7.36(\mathrm{~m}$, 2H), 7.35-7.30 (m, 3H), 7.07-6.99 (m, 2H), 6.35 (s, 1H), 3.86 (dd, $J=9.1,5.6 \mathrm{~Hz}, 1 \mathrm{H}), 2.50-2.39$ (m, 2H), 2.33 (s, 3H), 2.01-1.89 (m, 2H) ${ }^{13} \mathrm{C}$ NMR (151 MHz, $\left.\mathrm{CDCl}_{3}\right) \delta 161.8(\mathrm{~d}, J=245.1 \mathrm{~Hz})$, 137.0 (q, $J=3.2 \mathrm{~Hz}), 136.3$ (d, $J=3.0 \mathrm{~Hz}), 131.6,128.9$ (d, $J=7.9 \mathrm{~Hz}), 128.3,128.1,124.5$ (q, $J$ $=275.2 \mathrm{~Hz}), 123.3,123.1(\mathrm{q}, J=29.8 \mathrm{~Hz}), 115.4(\mathrm{~d}, J=21.4 \mathrm{~Hz}), 90.1,84.4,37.4,36.8,30.6(\mathrm{q}, J$ $=1.6 \mathrm{~Hz}), 18.5(\mathrm{q}, J=2.1 \mathrm{~Hz}) ;{ }^{19} \mathrm{~F}$ NMR $\left(565 \mathrm{MHz}, \mathrm{CDCl}_{3}\right) \delta-61.7,-116.0$; HRMS (APCI-TOF) calcd for $\mathrm{C}_{21} \mathrm{H}_{19} \mathrm{~F}_{4} \mathrm{~S}(\mathrm{M}+\mathrm{H})^{+} 379.1138$, found 379.1135 .<smiles>CC(=CC(CCC(C#Cc1ccccc1)c1ccc(Cl)cc1)C(F)(F)F)C(F)(F)F</smiles>

Compound 4j: $53 \mathrm{mg}, 67 \%$ yield, yellow oil; Flash column chromatography conditions: EtOAc/petroleum ethers = 1:100; ${ }^{1} \mathrm{H}$ NMR $\left(600 \mathrm{MHz}, \mathrm{CDCl}_{3}\right) \delta 7.46-7.42(\mathrm{~m}, 2 \mathrm{H}), 7.36-7.34(\mathrm{~m}$, 2H), 7.33-7.30 (m, 5H), $6.34(\mathrm{~s}, 1 \mathrm{H}), 3.85$ (dd, $J=9.1,5.6 \mathrm{~Hz}, 1 \mathrm{H}), 2.48-2.37$ (m, 2H), $2.32(\mathrm{~s}$, 3H), 1.99-1.90 (m, 2H); ${ }^{13} \mathrm{C}$ NMR (151 MHz, $\left.\mathrm{CDCl}_{3}\right) \delta 139.8,136.4$ (q, $\left.J=3.3 \mathrm{~Hz}\right), 132.7,131.6$, 128.8, 128.7, 128.3, 128.1, 124.5 (q, $J=275.2 \mathrm{~Hz}), 123.2,123.0$ (q, $J=29.7 \mathrm{~Hz}), 89.7,84.5,37.2$, $37.0,30.5$ (q, $J=1.9 \mathrm{~Hz}), 18.5(\mathrm{q}, J=2.2 \mathrm{~Hz}) ;{ }^{19} \mathrm{~F}$ NMR $\left(565 \mathrm{MHz}, \mathrm{CDCl}_{3}\right) \delta-61.7$; HRMS 
(APCI-TOF) calcd for $\mathrm{C}_{21} \mathrm{H}_{19} \mathrm{ClF}_{3} \mathrm{~S}(\mathrm{M}+\mathrm{H})^{+}$395.0843, found 395.0823.<smiles>C/C=C(\CCC(C#Cc1ccccc1)c1ccc(Br)cc1)C(F)(F)F</smiles>

Compound $4 \boldsymbol{k}$ : $61 \mathrm{mg}, 70 \%$ yield, yellow oil; Flash column chromatography conditions: EtOAc/petroleum ethers $=1: 100 ;{ }^{1} \mathrm{H}$ NMR $\left(600 \mathrm{MHz}, \mathrm{CDCl}_{3}\right) \delta 7.49-7.45(\mathrm{~m}, 2 \mathrm{H}), 7.45-7.41(\mathrm{~m}$, 2H), 7.35-7.28 (m, 5H), 6.34 (s, 1H), 3.83 (dd, $J=9.1,5.6 \mathrm{~Hz}, 1 \mathrm{H}), 2.49-2.38$ (m, 2H), $2.32(\mathrm{~s}$, 3H), 2.04-1.87 (m, 2H); ${ }^{13} \mathrm{C}$ NMR (151 MHz, $\left.\mathrm{CDCl}_{3}\right) \delta 140.3,136.4$ (q, $\left.J=3.2 \mathrm{~Hz}\right), 131.7,131.6$, 129.2, 128.3, 128.1, 124.5 (q, $J=275.0 \mathrm{~Hz}), 123.2,123.0$ (q, $J=29.8 \mathrm{~Hz}), 120.8,89.7,84.6,37.1$, $37.1,30.6(\mathrm{q}, J=1.6 \mathrm{~Hz}), 18.5(\mathrm{q}, J=2.1 \mathrm{~Hz}) ;{ }^{19} \mathrm{~F}$ NMR $\left(565 \mathrm{MHz}, \mathrm{CDCl}_{3}\right) \delta-61.7$; HRMS (APCI-TOF) calcd for $\mathrm{C}_{21} \mathrm{H}_{19} \mathrm{BrF}_{3} \mathrm{~S}(\mathrm{M}+\mathrm{H})^{+} 439.0337$, found 439.0327 .<smiles>CO/C=C(\CCC(C#Cc1ccccc1)c1cccs1)C(F)(F)F</smiles>

41

Compound 4l: $48 \mathrm{mg}, 65 \%$ yield, yellow oil; Flash column chromatography conditions: EtOAc/petroleum ethers $=1: 100 ;{ }^{1} \mathrm{H}$ NMR $\left(600 \mathrm{MHz}, \mathrm{CDCl}_{3}\right) \delta 7.46-7.44(\mathrm{~m}, 2 \mathrm{H}), 7.33-7.30(\mathrm{~m}$, 3H), $7.21(\mathrm{dd}, J=5.1,1.2 \mathrm{~Hz}, 1 \mathrm{H}), 7.04(\mathrm{dt}, J=3.4,1.0 \mathrm{~Hz}, 1 \mathrm{H}), 6.96(\mathrm{dd}, J=5.1,3.5 \mathrm{~Hz}, 1 \mathrm{H})$, $6.36(\mathrm{~s}, 1 \mathrm{H}), 4.17(\mathrm{dd}, J=8.5,5.5 \mathrm{~Hz}, 1 \mathrm{H}), 2.50-2.44(\mathrm{~m}, 2 \mathrm{H}), 2.33(\mathrm{~s}, 3 \mathrm{H}), 2.13-2.04(\mathrm{~m}, 2 \mathrm{H})$; ${ }^{13} \mathrm{C}$ NMR $\left(151 \mathrm{MHz}, \mathrm{CDCl}_{3}\right) \delta 144.8,136.4$ (q, $\left.J=3.2 \mathrm{~Hz}\right), 131.6,128.3,128.1,126.7,124.6$ (q, $J=275.0 \mathrm{~Hz}), 124.5,124.1,123.1,122.3(\mathrm{q}, J=30.0 \mathrm{~Hz}), 89.6,83.8,37.3,32.8,30.2(\mathrm{q}, J=2.0$ $\mathrm{Hz}), 18.5(\mathrm{q}, J=2.4 \mathrm{~Hz}) ;{ }^{19} \mathrm{~F}$ NMR $\left(565 \mathrm{MHz}, \mathrm{CDCl}_{3}\right) \delta-61.7$; MS (EI, $\left.m / z\right) 366\left(\mathrm{M}^{+}, 2\right), 351(26)$, 319 (36), 241 (39), 207 (22).<smiles>CC=C(COC(C#Cc1ccccc1)c1ccccc1)C(F)(F)F</smiles>

$4 m$ 
Compound 4m: $52 \mathrm{mg}, 72 \%$ yield, yellow oil; Flash column chromatography conditions: EtOAc/petroleum ethers $=1: 100 ;{ }^{1} \mathrm{H}$ NMR $\left(600 \mathrm{MHz}, \mathrm{CDCl}_{3}\right) \delta 7.51(\mathrm{~d}, J=7.4 \mathrm{~Hz}, 2 \mathrm{H}), 7.42(\mathrm{dd}$, $J=7.3,1.8 \mathrm{~Hz}, 2 \mathrm{H}), 7.33$ (t, $J=7.4 \mathrm{~Hz}, 2 \mathrm{H}), 7.28-7.24(\mathrm{~m}, 4 \mathrm{H}), 6.70(\mathrm{~s}, 1 \mathrm{H}), 5.39(\mathrm{~s}, 1 \mathrm{H}), 4.28$ $(\mathrm{d}, J=12.4 \mathrm{~Hz}, 1 \mathrm{H}), 4.19(\mathrm{~d}, J=12.4 \mathrm{~Hz}, 1 \mathrm{H}), 2.29(\mathrm{~s}, 3 \mathrm{H}) ;{ }^{13} \mathrm{C} \mathrm{NMR}\left(151 \mathrm{MHz}, \mathrm{CDCl}_{3}\right) \delta 141.2$ $(\mathrm{q}, J=2.9 \mathrm{~Hz}), 138.0,131.8,128.7,128.6,128.6,128.3,127.6,123.9(\mathrm{q}, J=274.5 \mathrm{~Hz}), 122.3$, 120.3 (q, $J=30.4 \mathrm{~Hz}), 88.4,86.1,71.1,66.9$ (q, $J=2.2 \mathrm{~Hz}), 18.6$ (q, $J=1.9 \mathrm{~Hz}) ;{ }^{19} \mathrm{~F}$ NMR $(565$ $\left.\mathrm{MHz}, \mathrm{CDCl}_{3}\right) \delta-61.7$; HRMS (APCI-TOF) calcd for $\mathrm{C}_{20} \mathrm{H}_{18} \mathrm{~F}_{3} \mathrm{OS}(\mathrm{M}+\mathrm{H})^{+} 363.1025$, found 363.1022 .<smiles>CC(=CCCOC(C#CPc1ccccc1)c1ccccc1)C(F)(F)F</smiles>

$4 n$

Compound 4n: $26 \mathrm{mg}, 34 \%$ yield, yellow oil; Flash column chromatography conditions: EtOAc/petroleum ethers $=1: 100 ;{ }^{1} \mathrm{H}$ NMR $\left(600 \mathrm{MHz}, \mathrm{CDCl}_{3}\right) \delta 7.58(\mathrm{~d}, J=7.3 \mathrm{~Hz}, 2 \mathrm{H})$, 7.49-7.47 (m, 2H), 7.40 (t, J=7.4 Hz, 3H), 7.33-7.25 (m, 3H), $6.46(\mathrm{~s}, 1 \mathrm{H}), 5.41$ (s, 1H), 3.85 (dt, $J=9.2,6.5 \mathrm{~Hz}, 1 \mathrm{H}), 3.68(\mathrm{dt}, J=9.2,6.6 \mathrm{~Hz}, 1 \mathrm{H}), 2.56-2.51(\mathrm{~m}, 2 \mathrm{H}), 2.29(\mathrm{~s}, 3 \mathrm{H}),{ }^{13} \mathrm{C} \mathrm{NMR}$ $\left(151 \mathrm{MHz}, \mathrm{CDCl}_{3}\right) \delta 141.2(\mathrm{q}, J=2.9 \mathrm{~Hz}), 138.0,131.8,128.7,128.6,128.6,128.3,127.6,123.9$ (q, $J=274.5 \mathrm{~Hz}), 122.3,120.3(\mathrm{q}, J=30.4 \mathrm{~Hz}), 88.4,86.1,71.1,66.9(\mathrm{q}, J=2.1 \mathrm{~Hz}), 29.7,18.5$ (q, $J=1.9 \mathrm{~Hz}) ;{ }^{19} \mathrm{~F}$ NMR (565 MHz, $\left.\mathrm{CDCl}_{3}\right) \delta-61.7$; HRMS (APCI-TOF) calcd for $\mathrm{C}_{21} \mathrm{H}_{20} \mathrm{~F}_{3} \mathrm{OS}$ $(\mathrm{M}+\mathrm{H})^{+} 377.1181$, found 377.1175 .<smiles>C/C(=C/CC(C#CC#Cc1ccccc1)(c1ccccc1)C(F)(F)F)C(F)(F)F</smiles>

Compound 4o: $55 \mathrm{mg}, 73 \%$ yield, yellow oil; Flash column chromatography conditions: EtOAc/petroleum ethers $=1: 100 ;{ }^{1} \mathrm{H}$ NMR $\left(600 \mathrm{MHz}, \mathrm{CDCl}_{3}\right) \delta 7.65(\mathrm{~d}, J=7.9 \mathrm{~Hz}, 2 \mathrm{H})$, 7.46-7.37 (m, 2H), 7.37-7.20 (m, 5H), 7.20-7.16 (m, 1H), 6.3 (s, 1H), 2.52-2.47 (m, 1H), 2.16 (s, 3H), 2.15-2.09 (m, 3H), 1.75 (s, 3H); ${ }^{13} \mathrm{C} \mathrm{NMR}\left(151 \mathrm{MHz}, \mathrm{CDCl}_{3}\right) \delta 144.5,135.3$ (q, $\left.J=3.2 \mathrm{~Hz}\right)$, 131.6, 128.4, 128.3, 127.9, 126.7, 126.0, 124.5 (q, $J=275.2 \mathrm{~Hz}), 124.2(\mathrm{q}, J=29.7 \mathrm{~Hz}), 123.5$, 
93.8, 84.7, 43.3, 41.0, 30.5 (q, $J=1.6 \mathrm{~Hz}), 29.1,18.4$ (q, $J=2.1 \mathrm{~Hz}) ;{ }^{19} \mathrm{~F}$ NMR $\left(565 \mathrm{MHz}, \mathrm{CDCl}_{3}\right)$ $\delta$-61.8; MS (EI, $m / z) 374\left(\mathrm{M}^{+}, 40\right), 359$ (15), 327 (6), 311 (10), 241 (22).<smiles>CC=C(CCC(C)(C)C#CPc1ccccc1)C(F)(F)F</smiles>

Compound 4p: $37 \mathrm{mg}$, 60\% yield, colorless oil; Flash column chromatography conditions: EtOAc/petroleum ethers $=1: 100 ;{ }^{1} \mathrm{H}$ NMR $\left(600 \mathrm{MHz}, \mathrm{CDCl}_{3}\right) \delta 7.41(\mathrm{dd}, J=7.8,1.7 \mathrm{~Hz}, 2 \mathrm{H})$, 7.28-7.24 (m, 3H), $6.71(\mathrm{~s}, 1 \mathrm{H}), 2.49-2.44(\mathrm{~m}, 2 \mathrm{H}), 2.37(\mathrm{~s}, 3 \mathrm{H}), 1.68-1.63(\mathrm{~m}, 2 \mathrm{H}), 1.32(\mathrm{~s}, 6 \mathrm{H})$; ${ }^{13} \mathrm{C} \mathrm{NMR}\left(151 \mathrm{MHz}, \mathrm{CDCl}_{3}\right) \delta 134.7$ (q, $\left.J=6.6 \mathrm{~Hz}\right), 131.6,128.1,127.5,124.8$ (q, $\left.J=29.6 \mathrm{~Hz}\right)$, $123.9,123.8$ (q, $J=272.2 \mathrm{~Hz}), 96.1,81.0,40.3,31.7,28.9,24.0,17.0 ;{ }^{19} \mathrm{~F}$ NMR $\left(565 \mathrm{MHz}, \mathrm{CDCl}_{3}\right)$ $\delta$-65.9; HRMS (APCI-TOF) calcd for $\mathrm{C}_{17} \mathrm{H}_{20} \mathrm{~F}_{3} \mathrm{~S}(\mathrm{M}+\mathrm{H})^{+} 313.1233$, found 313.1230 .<smiles>CC=C(CCC(C#CPc1ccccc1)Cc1ccccc1)C(F)(F)F</smiles>

Compound 4q: $22 \mathrm{mg}, 30 \%$ yield, yellow oil; Flash column chromatography conditions: EtOAc/petroleum ethers $=1: 100 ;{ }^{1} \mathrm{H}$ NMR $\left(600 \mathrm{MHz}, \mathrm{CDCl}_{3}\right) \delta 7.38-7.35(\mathrm{~m}, 2 \mathrm{H}), 7.32-7.22(\mathrm{~m}$, 8H), $6.72(\mathrm{~s}, 1 \mathrm{H}), 2.95-2.75(\mathrm{~m}, 3 \mathrm{H}), 2.64-2.56(\mathrm{~m}, 1 \mathrm{H}), 2.42-2.33(\mathrm{~m}, 4 \mathrm{H}), 1.83-1.77(\mathrm{~m}, 1 \mathrm{H})$, 1.74-1.67 (m, 1H); ${ }^{13} \mathrm{C}$ NMR (151 MHz, CDCl3) $\delta 139.3,135.3(\mathrm{q}, J=6.6 \mathrm{~Hz}), 131.5,129.3$, $128.2,128.1,127.6,126.3,124.5(\mathrm{q}, J=275.1 \mathrm{~Hz}), 123.1(\mathrm{q}, J=29.6 \mathrm{~Hz}), 91.8,83.3,41.2,34.6$, 32.0, 25.6, 17.1; ${ }^{19} \mathrm{~F}$ NMR (565 MHz, $\mathrm{CDCl}_{3}$ ) $\delta$-65.7; HRMS (APCI-TOF) calcd for $\mathrm{C}_{22} \mathrm{H}_{22} \mathrm{~F}_{3} \mathrm{~S}$ $(\mathrm{M}+\mathrm{H})^{+} 375.1389$, found 375.1379 .<smiles>CO/C=C(\CCC(C#Cc1ccccc1)Oc1ccc2c(C)cc(=O)oc2c1)C(F)(F)F</smiles>

Compound 4r: $32 \mathrm{mg}, 35 \%$ yield, yellow oil; Flash column chromatography conditions: EtOAc/petroleum ethers $=1: 20 ;{ }^{1} \mathrm{H}$ NMR $\left(600 \mathrm{MHz}, \mathrm{CDCl}_{3}\right) \delta 7.53(\mathrm{~d}, J=8.8 \mathrm{~Hz}, 1 \mathrm{H}), 7.40(\mathrm{~d}, J$ $=6.8 \mathrm{~Hz}, 2 \mathrm{H}), 7.36-7.31(\mathrm{~m}, 3 \mathrm{H}), 7.07(\mathrm{~d}, J=2.3 \mathrm{~Hz}, 1 \mathrm{H}), 7.00(\mathrm{dd}, J=8.8,2.3 \mathrm{~Hz}, 1 \mathrm{H}), 6.39(\mathrm{~s}$, 
1H), $6.18(\mathrm{~s}, 1 \mathrm{H}), 5.04(\mathrm{t}, J=6.3 \mathrm{~Hz}, 1 \mathrm{H}), 2.64-2.58(\mathrm{~m}, 2 \mathrm{H}), 2.41(\mathrm{~s}, 3 \mathrm{H}), 2.28(\mathrm{~s}, 3 \mathrm{H})$, 2.26-2.19 (m, 2H); ${ }^{13} \mathrm{C}$ NMR (151 MHz, $\left.\mathrm{CDCl}_{3}\right) \delta 161.2,160.4,155.0,152.4,137.0$ (q, $J=3.2$ Hz), 131.8, 129.0, 128.3, 125.5, 124.5 (q, $J=274.9 \mathrm{~Hz}), 122.4$ (q, $J=29.9 \mathrm{~Hz}), 121.6,114.1$, 113.1, 112.3, 102.9, 87.9, 85.2, 67.6, 34.6, 28.3, 18.7, 18.5 (q, $J=2.3 \mathrm{~Hz}) ;{ }^{19} \mathrm{~F}$ NMR (565 MHz, $\left.\mathrm{CDCl}_{3}\right) \delta-61.7$; HRMS (APCI-TOF) calcd for $\mathrm{C}_{25} \mathrm{H}_{22} \mathrm{~F}_{3} \mathrm{O}_{3} \mathrm{~S}(\mathrm{M}+\mathrm{H})^{+} 459.1236$, found 459.1239.<smiles>CC/C=C(\CCC(C#Cc1ccccc1)c1ccccc1)C(F)(F)F</smiles>

Compound 4s: $51 \mathrm{mg}, 68 \%$ yield, yellow oil; Flash column chromatography conditions: EtOAc/petroleum ethers $=1: 100 ;{ }^{1} \mathrm{H}$ NMR $\left(600 \mathrm{MHz}, \mathrm{CDCl}_{3}\right) \delta 7.47(\mathrm{dd}, J=6.5,3.2 \mathrm{~Hz}, 2 \mathrm{H})$, $7.44(\mathrm{~d}, J=7.3 \mathrm{~Hz}, 2 \mathrm{H}), 7.37(\mathrm{t}, J=7.7 \mathrm{~Hz}, 2 \mathrm{H}), 7.35-7.30(\mathrm{~m}, 3 \mathrm{H}), 7.28(\mathrm{t}, J=7.3 \mathrm{~Hz}, 1 \mathrm{H}), 6.42$ (s, 1H), 3.89 (dd, $J=9.3,5.5 \mathrm{~Hz}, 1 \mathrm{H}), 2.72(\mathrm{~d}, J=7.4 \mathrm{~Hz}, 2 \mathrm{H}), 2.55-2.44(\mathrm{~m}, 2 \mathrm{H}), 2.08-1.90(\mathrm{~m}$, $1 \mathrm{H}), 1.32(\mathrm{t}, J=7.4 \mathrm{~Hz}, 3 \mathrm{H}) ;{ }^{13} \mathrm{C} \mathrm{NMR}\left(151 \mathrm{MHz}, \mathrm{CDCl}_{3}\right) \delta 141.3,134.4$ (q, $\left.J=3.1 \mathrm{~Hz}\right), 131.6$, 128.6, 128.3, 128.0, 127.4, 126.9, $124.6(\mathrm{q}, J=275.1 \mathrm{~Hz}), 123.5,123.4(\mathrm{q}, J=29.9 \mathrm{~Hz}), 90.4$, 84.2, 37.5, 37.3, 30.7 (q, $J=1.8 \mathrm{~Hz}), 29.3$ (q, $J=1.9 \mathrm{~Hz}), 15.3 ;{ }^{19} \mathrm{~F}$ NMR $\left(565 \mathrm{MHz}, \mathrm{CDCl}_{3}\right) \delta$ -61.7; HRMS (APCI-TOF) calcd for $\mathrm{C}_{22} \mathrm{H}_{22} \mathrm{~F}_{3} \mathrm{~S}(\mathrm{M}+\mathrm{H})^{+} 375.1389$, found 375.1384 .<smiles>FC(F)(F)C(=Cc1ccccc1)CCC(C#Cc1ccccc1)c1ccccc1</smiles>

Compound 4t: $45 \mathrm{mg}, 53 \%$ yield, yellow oil; Flash column chromatography conditions: EtOAc/petroleum ethers $=1: 100 ;{ }^{1} \mathrm{H}$ NMR $\left(600 \mathrm{MHz}, \mathrm{CDCl}_{3}\right) \delta 7.48-7.27(\mathrm{~m}, 15 \mathrm{H}), 6.70(\mathrm{~s}, 1 \mathrm{H})$, $3.93(\mathrm{dd}, J=9.1,5.6 \mathrm{~Hz}, 1 \mathrm{H}), 2.58-2.53(\mathrm{~m}, 2 \mathrm{H}), 2.08-1.99(\mathrm{~m}, 2 \mathrm{H}) ;{ }^{13} \mathrm{C}$ NMR $(151 \mathrm{MHz}$, $\left.\mathrm{CDCl}_{3}\right) \delta 141.3,134.3(\mathrm{q}, J=2.9 \mathrm{~Hz}), 131.6,130.9,129.4,128.6,128.2,128.0,127.9,127.9$, 127.4, 127.0, 124.4 (q, $J=275.4 \mathrm{~Hz}), 124.1$ (q, $J=29.8 \mathrm{~Hz}), 123.3,90.2,84.4,37.5,37.1,30.7$ (q, $J=1.7 \mathrm{~Hz}) ;{ }^{19} \mathrm{~F}$ NMR $\left(565 \mathrm{MHz}, \mathrm{CDCl}_{3}\right) \delta-61.5$; HRMS (APCI-TOF) calcd for $\mathrm{C}_{26} \mathrm{H}_{22} \mathrm{~F}_{3} \mathrm{~S}(\mathrm{M}+$ H) ${ }^{+} 423.1389$, found 423.1381 . 
<smiles>COC=C(CCC(C#Cc1ccccc1)c1ccccc1)C(F)(F)F</smiles>

Compound $4 \boldsymbol{u}$ : $45 \mathrm{mg}, 55 \%$ yield, yellow oil; Flash column chromatography conditions: EtOAc/petroleum ethers $=1: 100 ;{ }^{1} \mathrm{H}$ NMR $\left(600 \mathrm{MHz}, \mathrm{CDCl}_{3}\right) \delta 7.45-7.41(\mathrm{~m}, 4 \mathrm{H}), 7.36-7.35(\mathrm{~m}$, 2H), 7.31-7.30 (m, 3H), 7.27 (d, $J=7.3 \mathrm{~Hz}, 1 \mathrm{H}), 6.67$ (s, 1H), 3.88 (dd, $J=9.0,5.6 \mathrm{~Hz}, 1 \mathrm{H})$, 2.52-2.41 (m, 2H), $2.18(\mathrm{~s}, 3 \mathrm{H}), 2.13-1.89(\mathrm{~m}, 2 \mathrm{H}) ;{ }^{13} \mathrm{C} \mathrm{NMR}\left(151 \mathrm{MHz}, \mathrm{CDCl}_{3}\right) \delta 141.3$, 131.6, $130.3(\mathrm{q}, J=3.7 \mathrm{~Hz}), 128.6,128.3,128.0,127.4,127.1(\mathrm{q}, J=29.7 \mathrm{~Hz}), 127.0,124.8(\mathrm{q}, J=275.2$ $\mathrm{Hz}), 123.4,90.4,84.2,37.6,37.0,31.2$ (q, $J=1.7 \mathrm{~Hz}), 8.5$ (q, $J=3.0 \mathrm{~Hz}) ;{ }^{19} \mathrm{~F}$ NMR $(565 \mathrm{MHz}$, $\left.\mathrm{CDCl}_{3}\right) \delta-62.7$; HRMS (APCI-TOF) calcd for $\mathrm{C}_{21} \mathrm{H}_{20} \mathrm{~F}_{3} \mathrm{Se}(\mathrm{M}+\mathrm{H})^{+} 409.0677$, found 409.0672 .<smiles>Cc1cccc(O/C=C(\CCC(C#Cc2ccccc2)c2ccccc2)C(F)(F)F)c1</smiles>

Compound 4v: $29 \mathrm{mg}, 34 \%$ yield, colorless oil; Flash column chromatography conditions: EtOAc/petroleum ethers $=1: 100 ;{ }^{1} \mathrm{H}$ NMR $\left(600 \mathrm{MHz}, \mathrm{CDCl}_{3}\right) \delta 7.44(\mathrm{~d}, J=7.1 \mathrm{~Hz}, 4 \mathrm{H}), 7.36(\mathrm{t}$, $J=7.6 \mathrm{~Hz}, 2 \mathrm{H}), 7.31-7.26(\mathrm{~m}, 4 \mathrm{H}), 7.20(\mathrm{t}, J=7.8 \mathrm{~Hz}, 1 \mathrm{H}), 6.93(\mathrm{~d}, J=7.6 \mathrm{~Hz}, 1 \mathrm{H}), 6.87-6.80$ (m, 2H), 6.68 (s, 1H), $3.93(\mathrm{dd}, J=9.6,5.4 \mathrm{~Hz}, 1 \mathrm{H}), 2.51-2.45(\mathrm{~m}, 1 \mathrm{H}), 2.43-2.40(\mathrm{~m}, 1 \mathrm{H}), 2.32$ (s, 3H), 2.05-2.00 (m, 1H), 1.99-1.97 (m, 1H); $\left.{ }^{13} \mathrm{C} \mathrm{NMR} \mathrm{(151} \mathrm{MHz,} \mathrm{CDCl}_{3}\right) \delta 156.7,145.9$ (q, $J$ $=6.9,3.5 \mathrm{~Hz}), 141.4,140.1,131.6,129.5,128.6,128.3,128.0,127.4,127.0,124.8,124.1(\mathrm{q}, J=$ $274.1 \mathrm{~Hz}), 123.4,117.7,113.9,110.6(\mathrm{q}, J=29.5 \mathrm{~Hz}), 90.3,84.3,37.5,37.4,25.9$ (q, $J=1.8 \mathrm{~Hz})$, 21.3; ${ }^{19} \mathrm{~F}$ NMR (565 MHz, $\left.\mathrm{CDCl}_{3}\right) \delta$-60.0; HRMS (ESI) calcd for $\mathrm{C}_{27} \mathrm{H}_{24} \mathrm{~F}_{3} \mathrm{O}(\mathrm{M}+\mathrm{H}){ }^{+} 421.1774$, found 421.1774. 


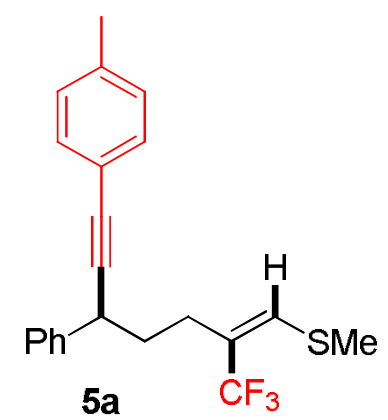

Compound 5a: $57 \mathrm{mg}, 76 \%$ yield, light yellow oil; Flash column chromatography conditions: EtOAc/petroleum ethers $=1: 100 ;{ }^{1} \mathrm{H}$ NMR $\left(600 \mathrm{MHz}, \mathrm{CDCl}_{3}\right) \delta 7.41(\mathrm{~d}, J=7.5 \mathrm{~Hz}, 2 \mathrm{H})$, $7.39-7.32(\mathrm{~m}, 4 \mathrm{H}), 7.25(\mathrm{~d}, J=6.8 \mathrm{~Hz}, 1 \mathrm{H}), 7.12(\mathrm{t}, J=7.4 \mathrm{~Hz}, 2 \mathrm{H}), 6.33(\mathrm{~s}, 1 \mathrm{H}), 3.86(\mathrm{dd}, J=$ 9.1, 5.5 Hz, 1H), 2.46-2.36 (m, 2H), $2.34(\mathrm{~s}, 3 \mathrm{H}), 2.31(\mathrm{~s}, 3 \mathrm{H}), 2.02-1.90(\mathrm{~m}, 2 \mathrm{H}) ;{ }^{13} \mathrm{C}$ NMR $(151$ $\left.\mathrm{MHz}, \mathrm{CDCl}_{3}\right) \delta 141.5,138.0,136.1(\mathrm{q}, J=3.2 \mathrm{~Hz}), 131.5,129.0,128.6,127.4,126.9,124.5(\mathrm{q}, J$ $=275.0 \mathrm{~Hz}), 123.3(\mathrm{q}, J=29.6 \mathrm{~Hz}), 120.4,89.6,84.3,37.6,37.3,30.6(\mathrm{q}, J=1.7 \mathrm{~Hz}), 21.4,18.5$ $(\mathrm{q}, J=2.1 \mathrm{~Hz}) ;{ }^{19} \mathrm{~F}$ NMR $\left(565 \mathrm{MHz}, \mathrm{CDCl}_{3}\right) \delta-61.7$; HRMS (APCI-TOF) calcd for $\mathrm{C}_{22} \mathrm{H}_{22} \mathrm{~F}_{3} \mathrm{~S}(\mathrm{M}$ $+\mathrm{H})^{+} 375.1389$, found 375.1387 .<smiles>CC(C)(C)C=C(CCC(C#Cc1ccc(C(C)(C)C)cc1)c1ccccc1)C(C)(C)C</smiles>

Compound $\mathbf{5 b}$ : $58 \mathrm{mg}$, 70\% yield, light yellow oil; Flash column chromatography conditions: EtOAc/petroleum ethers $=1: 100 ;{ }^{1} \mathrm{H}$ NMR $\left(600 \mathrm{MHz}, \mathrm{CDCl}_{3}\right) \delta 7.42(\mathrm{~d}, J=7.3 \mathrm{~Hz}, 2 \mathrm{H})$, 7.35-7.33 (m, 4H), 7.26-7.24 (m, 1H), 7.13-7.08 (m, 2H), $6.34(\mathrm{~s}, 1 \mathrm{H}), 3.87(\mathrm{dd}, J=9.2,5.4 \mathrm{~Hz}$, 1H), 2.51-2.41 (m, 2H), $2.32(\mathrm{~s}, 3 \mathrm{H}), 2.01-1.92(\mathrm{~m}, 2 \mathrm{H}), 1.31(\mathrm{~s}, 9 \mathrm{H}) ;{ }^{13} \mathrm{C}$ NMR $(151 \mathrm{MHz}$, $\left.\mathrm{CDCl}_{3}\right) \delta 151.2,141.46,136.1(\mathrm{q}, J=3.2 \mathrm{~Hz}), 131.3,128.6,127.4,126.9,125.3,124.6(\mathrm{q}, J=$ $275.1 \mathrm{~Hz}), 123.3(\mathrm{q}, J=29.6 \mathrm{~Hz}), 120.4,89.6,84.3,37.6,37.4,34.7,31.2,30.6(\mathrm{q}, J=1.7 \mathrm{~Hz})$, $18.5(\mathrm{q}, J=2.1 \mathrm{~Hz}) ;{ }^{19} \mathrm{~F}$ NMR $\left(565 \mathrm{MHz}, \mathrm{CDCl}_{3}\right) \delta-61.7$; HRMS (APCI-TOF) calcd for $\mathrm{C}_{25} \mathrm{H}_{28} \mathrm{~F}_{3} \mathrm{~S}(\mathrm{M}+\mathrm{H})^{+} 417.1858$, found 417.1854 . 


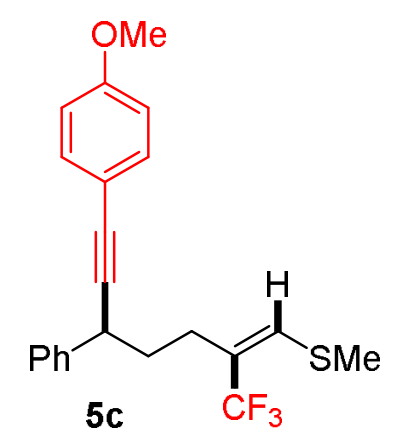

Compound 5c: $45 \mathrm{mg}, 57 \%$ yield, yellow oil; Flash column chromatography conditions: EtOAc/petroleum ethers $=1: 100 ;{ }^{1} \mathrm{H}$ NMR $\left(600 \mathrm{MHz}, \mathrm{CDCl}_{3}\right) \delta 7.44(\mathrm{dd}, J=6.5,3.0 \mathrm{~Hz}, 2 \mathrm{H})$, 7.34-7.29 (m, 5H), $6.89(\mathrm{~d}, J=8.6 \mathrm{~Hz}, 2 \mathrm{H}), 6.33(\mathrm{~s}, 1 \mathrm{H}), 3.84-3.79(\mathrm{~m}, 4 \mathrm{H}), 2.47-2.41(\mathrm{~m}, 2 \mathrm{H})$, 2.32 (s, 3H), 2.00-1.91 (m, 2H); ${ }^{13} \mathrm{C}$ NMR (151 MHz, $\left.\mathrm{CDCl}_{3}\right) \delta 158.5,136.0$ (q, $\left.J=3.1 \mathrm{~Hz}\right)$, 133.4, 131.6, 128.4, 128.3, 127.9, $124.5(\mathrm{q}, J=275.3 \mathrm{~Hz}) 123.5,123.3(\mathrm{q}, J=29.6 \mathrm{~Hz}), 114.0$, 90.8, 84.0, 55.3, 37.4, 36.7, 30.5 (q, $J=1.6 \mathrm{~Hz}), 18.5(\mathrm{q}, J=2.1 \mathrm{~Hz}) ;{ }^{19} \mathrm{~F}$ NMR $\left(565 \mathrm{MHz}, \mathrm{CDCl}_{3}\right)$ $\delta$-61.7; HRMS (APCI-TOF) calcd for $\mathrm{C}_{22} \mathrm{H}_{22} \mathrm{~F}_{3} \mathrm{OS}(\mathrm{M}+\mathrm{H})^{+} 391.1338$, found 391.1337 .<smiles>CC(=CC(CCC(C#Cc1ccc(F)cc1)c1ccccc1)C(F)(F)F)C(F)(F)F</smiles>

Compound 5d: $55 \mathrm{mg}, 73 \%$ yield, yellow oil; Flash column chromatography conditions: EtOAc/petroleum ethers $=1: 100 ;{ }^{1} \mathrm{H}$ NMR $\left(600 \mathrm{MHz}, \mathrm{CDCl}_{3}\right) \delta 7.44-7.39(\mathrm{~m}, 4 \mathrm{H}), 7.38-7.34(\mathrm{~m}$, 2H), 7.28-7.25 (m, 1H), 7.02-6.98 (m, 2H), $6.31(\mathrm{~s}, 1 \mathrm{H}), 3.85(\mathrm{dd}, J=8.9,5.8 \mathrm{~Hz}, 1 \mathrm{H}), 2.50-2.44$ (m, 1H), 2.44-2.38 (m, 1H), $2.32(\mathrm{~s}, 3 \mathrm{H}), 2.01-1.93(\mathrm{~m}, 2 \mathrm{H}) ;{ }^{13} \mathrm{C} \mathrm{NMR}\left(151 \mathrm{MHz}, \mathrm{CDCl}_{3}\right) \delta$ $162.3(\mathrm{~d}, J=249.0 \mathrm{~Hz}), 141.2,136.1(\mathrm{q}, J=3.3 \mathrm{~Hz}), 133.4(\mathrm{~d}, J=8.3 \mathrm{~Hz}), 128.7,127.4,127.0$, 124.5 (q, $J=275.2 \mathrm{~Hz}), 123.2$ (q, $J=29.7 \mathrm{~Hz}), 119.5$ (d, $J=3.6 \mathrm{~Hz}), 115.5$ (d, $J=22.0 \mathrm{~Hz}), 90.1$ $(\mathrm{d}, J=1.5 \mathrm{~Hz}), 83.0,37.6,37.2,30.5$ (q, $J=1.9 \mathrm{~Hz}), 18.5$ (q, $J=2.2 \mathrm{~Hz}) ;{ }^{19} \mathrm{~F}$ NMR $(565 \mathrm{MHz}$, $\left.\mathrm{CDCl}_{3}\right) \delta$-61.7, -111.5; HRMS (APCI-TOF) calcd for $\mathrm{C}_{21} \mathrm{H}_{19} \mathrm{~F}_{4} \mathrm{~S}(\mathrm{M}+\mathrm{H})^{+} 379.1138$, found 379.1127. 


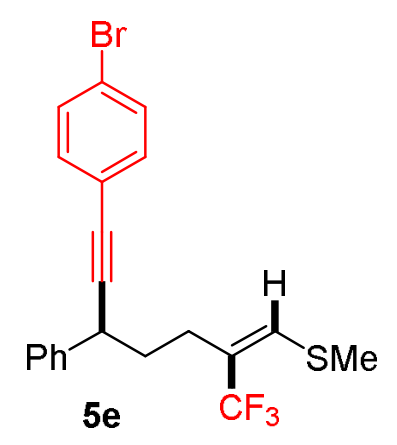

Compound 5e: $60 \mathrm{mg}$, 68\% yield, yellow oil; Flash column chromatography conditions: EtOAc/petroleum ethers = 1:100; ${ }^{1} \mathrm{H}$ NMR $\left(600 \mathrm{MHz}, \mathrm{CDCl}_{3}\right) \delta 7.43-7.41(\mathrm{~m}, 2 \mathrm{H}), 7.40(\mathrm{dd}, J=$ 5.7, $2.4 \mathrm{~Hz}, 2 \mathrm{H}), 7.38-7.33(\mathrm{~m}, 2 \mathrm{H}), 7.33-7.25(\mathrm{~m}, 3 \mathrm{H}), 6.31(\mathrm{~s}, 1 \mathrm{H}), 3.94-3.79(\mathrm{~m}, 1 \mathrm{H})$, 2.51-2.37 (m, 2H), 2.31 (s, 3H), 2.31-1.98 (m, 2H); ${ }^{13} \mathrm{C}$ NMR (151 MHz, $\left.\mathrm{CDCl}_{3}\right) \delta 140.9,136.2$ $(\mathrm{q}, J=3.1 \mathrm{~Hz}), 133.0,131.5,128.6,127.4,127.0,125.4,124.5$ (q, $J=275.2 \mathrm{~Hz}), 123.1(\mathrm{q}, J=$ $29.7 \mathrm{~Hz}), 122.2,91.7,83.0,37.6,37.1,30.5$ (q, $J=1.7 \mathrm{~Hz}), 18.4$ (q, $J=2.2 \mathrm{~Hz}) ;{ }^{19} \mathrm{~F}$ NMR $(565$ $\left.\mathrm{MHz}, \mathrm{CDCl}_{3}\right) \delta$-61.7; MS (EI, $\left.m / z\right) 440\left(\mathrm{M}^{+},{ }^{81} \mathrm{Br}, 2\right), 438\left(\mathrm{M}^{+},{ }^{79} \mathrm{Br}, 2\right), 425$ (26), 423 (24), 393 (11), 391 (11), 321 (43).<smiles>CC(=CC(CCC(C#Cc1ccc(Cl)cc1)c1ccccc1)C(F)(F)F)C(F)(F)F</smiles>

Compound 5f: $55 \mathrm{mg}, 70 \%$ yield, yellow oil; Flash column chromatography conditions: EtOAc/petroleum ethers $=1: 100 ;{ }^{1} \mathrm{H}$ NMR $\left(600 \mathrm{MHz}, \mathrm{CDCl}_{3}\right) \delta 7.39(\mathrm{~d}, J=7.2 \mathrm{~Hz}, 2 \mathrm{H})$, 7.37-7.34 (m, 4H), 7.29-7.26 (m, 3H), 6.31 (s, 1H), 3.85 (dd, $J=8.7,5.9 \mathrm{~Hz}, 1 \mathrm{H}), 2.48-2.43$ (m, $1 \mathrm{H}), 2.42-2.37(\mathrm{~m}, 1 \mathrm{H}), 2.30(\mathrm{~s}, 3 \mathrm{H}), 2.01-1.94(\mathrm{~m}, 2 \mathrm{H}) ;{ }^{13} \mathrm{C} \mathrm{NMR}\left(151 \mathrm{MHz}, \mathrm{CDCl}_{3}\right) \delta 141.0$, $136.2(\mathrm{q}, J=3.1 \mathrm{~Hz}), 133.9,132.8,128.6,128.6,127.4,127.0,124.5(\mathrm{q}, J=275.2 \mathrm{~Hz}), 123.1(\mathrm{q}, J$ $=29.8 \mathrm{~Hz}), 121.9,91.5,83.0,37.6,37.1,30.5(\mathrm{q}, J=1.6 \mathrm{~Hz}), 18.4(\mathrm{q}, J=2.1 \mathrm{~Hz}) ;{ }^{19} \mathrm{~F} \operatorname{NMR}(565$ $\left.\mathrm{MHz}, \mathrm{CDCl}_{3}\right) \delta-61.7$; MS (EI, m/z) $396\left(\mathrm{M}^{+},{ }^{37} \mathrm{Cl}, 1\right), 394\left(\mathrm{M}^{+},{ }^{35} \mathrm{Cl}, 3\right), 379$ (40), 347 (41), 312 (27), 275 (64). 


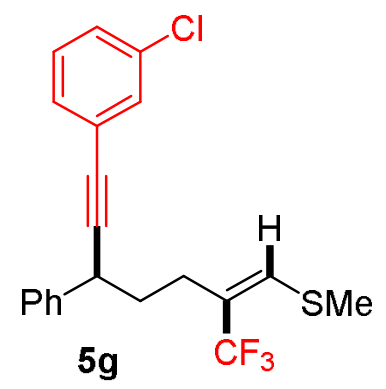

Compound 5g: $58 \mathrm{mg}, 73 \%$ yield, yellow oil; Flash column chromatography conditions: EtOAc/petroleum ethers $=1: 100 ;{ }^{1} \mathrm{H} \mathrm{NMR}\left(600 \mathrm{MHz}, \mathrm{CDCl}_{3}\right) \delta 7.43(\mathrm{~s}, 1 \mathrm{H}), 7.39(\mathrm{~d}, J=7.4 \mathrm{~Hz}$, 2H), 7.37-7.30 (m, 3H), 7.30-7.23 (m, 3H), $6.32(\mathrm{~s}, 1 \mathrm{H}), 3.86(\mathrm{dd}, J=8.5,6.1 \mathrm{~Hz}, 1 \mathrm{H}), 2.48-2.37$ $(\mathrm{m}, 2 \mathrm{H}), 2.32(\mathrm{~s}, 3 \mathrm{H}), 2.03-1.94(\mathrm{~m}, 2 \mathrm{H}) ;{ }^{13} \mathrm{C} \mathrm{NMR}\left(151 \mathrm{MHz}, \mathrm{CDCl}_{3}\right) \delta 140.9,136.2(\mathrm{q}, J=3.0$ Hz), 134.1, 131.5, 129.7, 129.5, 128.7, 128.3, 127.4, 127.1, 125.1, 124.5 (q, $J=275.2 \mathrm{~Hz}), 123.1$ $(\mathrm{q}, J=29.7 \mathrm{~Hz}), 91.9,82.8,37.6,37.1,30.6(\mathrm{q}, J=1.7 \mathrm{~Hz}), 18.5(\mathrm{q}, J=2.1 \mathrm{~Hz}) ;{ }^{19} \mathrm{~F}$ NMR $(565$ $\left.\mathrm{MHz}, \mathrm{CDCl}_{3}\right) \delta$-61.7; HRMS (APCI-TOF) calcd for $\mathrm{C}_{21} \mathrm{H}_{19} \mathrm{ClF}_{3} \mathrm{~S}(\mathrm{M}+\mathrm{H})^{+}$395.0843, found 395.0838.<smiles>CC(=CCC(c1ccccc1)C(C#Cc1ccccc1Cl)(c1ccccc1)C(F)(F)F)C(F)(F)F</smiles>

Compound $\mathbf{5 h}$ : $57 \mathrm{mg}$, 72\% yield, yellow oil; Flash column chromatography conditions: EtOAc/petroleum ethers $=1: 100 ;{ }^{1} \mathrm{H}$ NMR $\left(600 \mathrm{MHz}, \mathrm{CDCl}_{3}\right) \delta 7.49-7.44(\mathrm{~m}, 3 \mathrm{H}), 7.41(\mathrm{~d}, J=$ $7.9 \mathrm{~Hz}, 1 \mathrm{H}), 7.36(\mathrm{t}, J=7.6 \mathrm{~Hz}, 2 \mathrm{H}), 7.24-7.19(\mathrm{~m}, 3 \mathrm{H}), 6.42(\mathrm{~s}, 1 \mathrm{H}), 3.94(\mathrm{dd}, J=9.5,5.1 \mathrm{~Hz}$, 1H), 2.58-2.50 (m, 2H), $2.33(\mathrm{~s}, 3 \mathrm{H}), 2.06-1.96(\mathrm{~m}, 2 \mathrm{H}) ;{ }^{13} \mathrm{C} \mathrm{NMR}\left(151 \mathrm{MHz}, \mathrm{CDCl}_{3}\right) \delta 141.0$, $136.3(\mathrm{q}, J=3.2 \mathrm{~Hz}), 135.9,133.3,129.2,129.0,128.6,127.5,127.0,126.4,124.6(\mathrm{q}, J=275.1$ $\mathrm{Hz}), 123.3,123.1(\mathrm{q}, J=29.7 \mathrm{~Hz}), 95.9,81.2,37.7,37.4,30.6(\mathrm{q}, J=1.7 \mathrm{~Hz}), 18.5(\mathrm{q}, J=2.2 \mathrm{~Hz})$; ${ }^{19} \mathrm{~F}$ NMR (565 MHz, $\left.\mathrm{CDCl}_{3}\right) \delta-61.7$; HRMS (APCI-TOF) calcd for $\mathrm{C}_{21} \mathrm{H}_{19} \mathrm{ClF}_{3} \mathrm{~S}(\mathrm{M}+\mathrm{H})^{+}$ 395.0843, found 395.0829 . 
<smiles>CC(=CCCC(C#Cc1ccc(C(F)(F)F)cc1)C(F)(F)F)C(F)(F)F</smiles>

Compound 5i: $52 \mathrm{mg}, 61 \%$ yield, light yellow oil; Flash column chromatography conditions: EtOAc/petroleum ethers $=1: 100 ;{ }^{1} \mathrm{H}$ NMR $\left(600 \mathrm{MHz}, \mathrm{CDCl}_{3}\right) \delta 7.59-7.57(\mathrm{~m}, 4 \mathrm{H}), 7.56-7.54(\mathrm{~m}$, 2H), 7.42-7.41 (m, 2H), 7.39-7.27 (m, 1H), $6.32(\mathrm{~s}, 1 \mathrm{H}), 3.89(\mathrm{dd}, J=8.5,6.1 \mathrm{~Hz}, 1 \mathrm{H}), 2.48-2.47$ (m, 1H), 2.46-2.42 (m, 1H), $2.33(\mathrm{~s}, 3 \mathrm{H}), 2.04-1.96(\mathrm{~m}, 2 \mathrm{H}) ;{ }^{13} \mathrm{C}$ NMR $\left(151 \mathrm{MHz}, \mathrm{CDCl}_{3}\right) \delta$ $140.8,136.3(\mathrm{q}, J=3.2 \mathrm{~Hz}), 131.9,128.7,128.4,128.3,127.4,127.2,125.2(\mathrm{q}, J=3.8 \mathrm{~Hz}), 124.5$ $(\mathrm{q}, J=275.0 \mathrm{~Hz}), 124.0(\mathrm{q}, J=271.3 \mathrm{~Hz}), 123.1(\mathrm{q}, J=30.0 \mathrm{~Hz}), 123.0,93.2,82.9,37.6,37.1$, $30.5(\mathrm{q}, J=1.8 \mathrm{~Hz}), 18.5(\mathrm{q}, J=2.1 \mathrm{~Hz}) ;{ }^{19} \mathrm{~F}$ NMR (565 MHz, $\left.\mathrm{CDCl}_{3}\right) \delta-61.8,-62.8$; HRMS (APCI-TOF) calcd for $\mathrm{C}_{22} \mathrm{H}_{19} \mathrm{~F}_{6} \mathrm{~S}(\mathrm{M}+\mathrm{H})^{+} 429.1106$, found 429.1103 .<smiles>CC=C(CCC(C#C[13CH3])c1ccccc1)C(F)(F)F</smiles>

Compound 5j: $49 \mathrm{mg}$, 61\% yield, colorless oil; Flash column chromatography conditions: EtOAc/petroleum ethers $=1: 100 ;{ }^{1} \mathrm{H}$ NMR $\left(600 \mathrm{MHz}, \mathrm{CDCl}_{3}\right) \delta 7.35-7.31(\mathrm{~m}, 4 \mathrm{H}), 7.25-7.22(\mathrm{~m}$, 1H), 6.32 (s, 1H), 3.69 (dd, $J=9.6,5.0 \mathrm{~Hz}, 1 \mathrm{H}), 2.48-2.36(\mathrm{~m}, 2 \mathrm{H}), 2.33$ (s, 3H), 1.95-1.78 (m, 2H), 0.97 (s, 9H), 0.13 (s, 6H); ${ }^{13} \mathrm{C} \mathrm{NMR}\left(151 \mathrm{MHz}, \mathrm{CDCl}_{3}\right) \delta 141.1,136.0$ (q, J=3.1 Hz), 128.5, $127.3(\mathrm{q}, J=16.4 \mathrm{~Hz}), 126.8,124.5(\mathrm{q}, J=275.0 \mathrm{~Hz}), 123.2(\mathrm{q}, J=29.8 \mathrm{~Hz}), 107.7,86.7,37.9$, $37.5,30.5(\mathrm{q}, J=1.6 \mathrm{~Hz}), 26.1,18.5(\mathrm{q}, J=2.1 \mathrm{~Hz}), 16.6 ;{ }^{19} \mathrm{~F}$ NMR $\left(565 \mathrm{MHz}, \mathrm{CDCl}_{3}\right) \delta-61.8$; MS (EI, $m / z) 398\left(\mathrm{M}^{+}, 1\right), 383$ (16), 341 (65), 245 (32), 210 (18).

\section{Experimental Procedure for the Synthesis of $6 a^{3}$}

\footnotetext{
${ }^{3}$ Zhang, H.; Sun, N.; Hu, B.; Jin, L. Org. Chem. Front. 2019, 12, 1983.
} 
<smiles>CC(C)=CCCC(C#Cc1ccccc1)C(C)(C)C</smiles><smiles>CC(=CC(CCC(=Cc1ccccc1)c1ccccc1)C(F)(F)F)C(F)(F)F</smiles>

To a Schlenk flask containing $4 a(36.0 \mathrm{mg}, 0.1 \mathrm{mmol})$ and TBAB $(3.2 \mathrm{mg}, 0.01 \mathrm{mmol})$ in $2 \mathrm{~mL}$ of toluene was added $\mathrm{KOH}(5.6 \mathrm{mg}, 0.1 \mathrm{mmol})$. After stirring at $25{ }^{\circ} \mathrm{C}$ for $12 \mathrm{~h}$, the reaction mixture was concentrated, and the residue was purified by silica gel column chromatography $($ EtOAc/petroleum ethers $=1: 100)$ to give $27 \mathrm{mg}(75 \%$ yield $)$ of $\mathbf{6 a}$ as a colorless oil; ${ }^{1} \mathrm{H}$ NMR $\left(600 \mathrm{MHz}, \mathrm{CDCl}_{3}\right) \delta 7.43(\mathrm{~d}, J=7.4 \mathrm{~Hz}, 2 \mathrm{H}), 7.36-7.30(\mathrm{~m}, 6 \mathrm{H}), 7.24(\mathrm{t}, J=7.0 \mathrm{~Hz}, 2 \mathrm{H}), 6.57$ (t, $J=3.0 \mathrm{~Hz}, 1 \mathrm{H}), 6.26(\mathrm{~s}, 1 \mathrm{H}), 2.80-2.70(\mathrm{~m}, 2 \mathrm{H}), 2.56-2.44(\mathrm{~m}, 2 \mathrm{H}), 2.22(\mathrm{~s}, 3 \mathrm{H}) ;{ }^{13} \mathrm{C} \mathrm{NMR}(151$ $\left.\mathrm{MHz}, \mathrm{CDCl}_{3}\right) \delta 206.3,136.6(\mathrm{q}, J=3.3 \mathrm{~Hz}), 135.6,134.1,128.8,128.6,127.3,127.3,126.9$, 126.0, 124.6 (q, $J=275.0 \mathrm{~Hz}), 123.2(\mathrm{q}, J=29.7 \mathrm{~Hz}), 108.7,98.6,31.0$ (q, $J=1.8 \mathrm{~Hz}), 29.4,18.3$ $(\mathrm{q}, J=2.3 \mathrm{~Hz}),{ }^{19} \mathrm{~F}$ NMR $\left(565 \mathrm{MHz}, \mathrm{CDCl}_{3}\right) \delta-61.7$; HRMS (ESI) calcd for $\mathrm{C}_{21} \mathrm{H}_{20} \mathrm{~F}_{3} \mathrm{~S}(\mathrm{M}+\mathrm{H})^{+}$ 361.1232, found 361.1226 .

\section{Experimental Procedure for the Synthesis of $6 b^{4}$}<smiles>COc1ccccc1</smiles><smiles>CC(=CC(CCC(c1ccccc1)c1ccccc1)C(F)(F)F)C(F)(F)F</smiles>

To a Schlenk flask containing $\mathbf{5 j}$ (79.6 mg, $0.2 \mathrm{mmol})$ in $1 \mathrm{~mL}$ of THF was added TBAF (1.0 M in THF, $300 \mu \mathrm{L}, 0.3 \mathrm{mmol}$ ) at $0{ }^{\circ} \mathrm{C}$. After stirring at room temperature for $12 \mathrm{~h}$, the reaction mixture was quenched by water, extracted with EtOAc, dried over anhydrous $\mathrm{Na}_{2} \mathrm{SO}_{4}$, and concentrated. Column chromatography on silica gel (EtOAc/petroleum ethers $=1: 300)$ gave $51 \mathrm{mg}$ of terminal alkyne as a colorless oil, which was directly used for the next step.

To a mixture of the terminal alkyne obtained above $(51.2 \mathrm{mg}, 0.18 \mathrm{mmol})$ and $\mathrm{CuTc}(4.0 \mathrm{mg}$, $0.018 \mathrm{mmol})$ in $2 \mathrm{~mL}$ of toluene was slowly added $\mathrm{TsN}_{3}(35.5 \mathrm{mg}, 0.18 \mathrm{mmol})$ at $0{ }^{\circ} \mathrm{C}$. After stirring at room temperature overnight, the reaction mixture was quenched by saturated aqueous

\footnotetext{
${ }^{4}$ Raushel, J.; Fokin, V. V. Org. Lett. 2010, 12, 4952.
} 
$\mathrm{NH}_{4} \mathrm{Cl}$ solution, extracted with $\mathrm{DCM}$, dried over anhydrous $\mathrm{Na}_{2} \mathrm{SO}_{4}$, and concentrated. Column chromatography on silica gel (EtOAc/petroleum ethers $=10: 1)$ gave $75 \mathrm{mg}(78 \%$ yield over 2 steps) of $\mathbf{6 b}$ as a colorless oil. ${ }^{1} \mathrm{H}$ NMR $\left(600 \mathrm{MHz}, \mathrm{CDCl}_{3}\right) \delta 7.84(\mathrm{~d}, J=8.3 \mathrm{~Hz}, 3 \mathrm{H}), 7.41-7.38$ (m, 4H), 7.33 (t, $J=7.7 \mathrm{~Hz}, 2 \mathrm{H}), 7.22(\mathrm{t}, J=7.3 \mathrm{~Hz}, 1 \mathrm{H}), 6.28(\mathrm{~s}, 1 \mathrm{H}), 5.11(\mathrm{t}, J=3.2 \mathrm{~Hz}, 2 \mathrm{H})$, 2.61-2.57 (m, 2H), $2.48(\mathrm{~s}, 3 \mathrm{H}), 2.45-2.42(\mathrm{~m}, 2 \mathrm{H}), 2.32(\mathrm{~s}, 3 \mathrm{H}) ;{ }^{13} \mathrm{C} \mathrm{NMR}\left(151 \mathrm{MHz}, \mathrm{CDCl}_{3}\right) \delta$ $146.2,136.1(\mathrm{q}, J=3.2 \mathrm{~Hz}), 135.7,135.5,130.2,128.5,127.5,126.8,125.9,124.6(\mathrm{q}, J=275.0$ $\mathrm{Hz}), 123.4(\mathrm{q}, J=29.8 \mathrm{~Hz}), 103.7,78.8,30.8(\mathrm{q}, J=1.9 \mathrm{~Hz}), 28.9,21.7,18.5(\mathrm{q}, J=2.2 \mathrm{~Hz}) ;{ }^{19} \mathrm{~F}$ NMR (565 MHz, $\left.\mathrm{CDCl}_{3}\right) \delta$-61.8; HRMS (APCI-TOF) calcd for $\mathrm{C}_{22} \mathrm{H}_{23} \mathrm{~F}_{3} \mathrm{~N}_{3} \mathrm{O}_{2} \mathrm{~S}_{2}(\mathrm{M}+\mathrm{H})^{+}$ 482.1179, found 482.1178 .

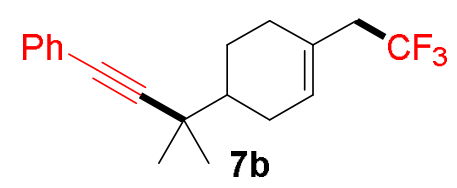

Compound $7 \boldsymbol{b}^{5}$ : $46 \mathrm{mg}, 75 \%$ yield, colorless oil; Flash column chromatography conditions: EtOAc/petroleum ethers = 1:100; ${ }^{1} \mathrm{H}$ NMR $\left(600 \mathrm{MHz}, \mathrm{CDCl}_{3}\right): \delta 7.39-7.34(\mathrm{~m}, 2 \mathrm{H}), 7.29-7.21(\mathrm{~m}$, 3H), 5.69 (s, 1H), 2.71 (q, $J=11.4 \mathrm{~Hz}, 2 \mathrm{H}), 2.31-1.98(\mathrm{~m}, 5 \mathrm{H}), 1.55-1.37$ (m, 2H), $1.29(\mathrm{~s}, 3 \mathrm{H})$, $1.26(\mathrm{~s}, 3 \mathrm{H}) ;{ }^{13} \mathrm{C} \mathrm{NMR}\left(151 \mathrm{MHz}, \mathrm{CDCl}_{3}\right): \delta 131.6,128.9,128.1,127.5,127.4$ (q, $\left.J=2.6 \mathrm{~Hz}\right)$, 126.2 (q, $J=278.7 \mathrm{~Hz}), 124.0,96.1,81.2,43.3,41.7$ (q, $J=28.9 \mathrm{~Hz}), 34.6,29.7,27.8,27.2,27.0$, 24.6; ${ }^{19} \mathrm{~F}$ NMR (565 MHz, $\left.\mathrm{CDCl}_{3}\right): \delta-64.8(\mathrm{t}, J=11.2 \mathrm{~Hz})$.

\footnotetext{
${ }^{5}$ Zhou, S.; Song, T.; Chen, H.; Liu, Z.; Shen, H.; Li, C. Org. Lett. 2017, 19, 698.
} 

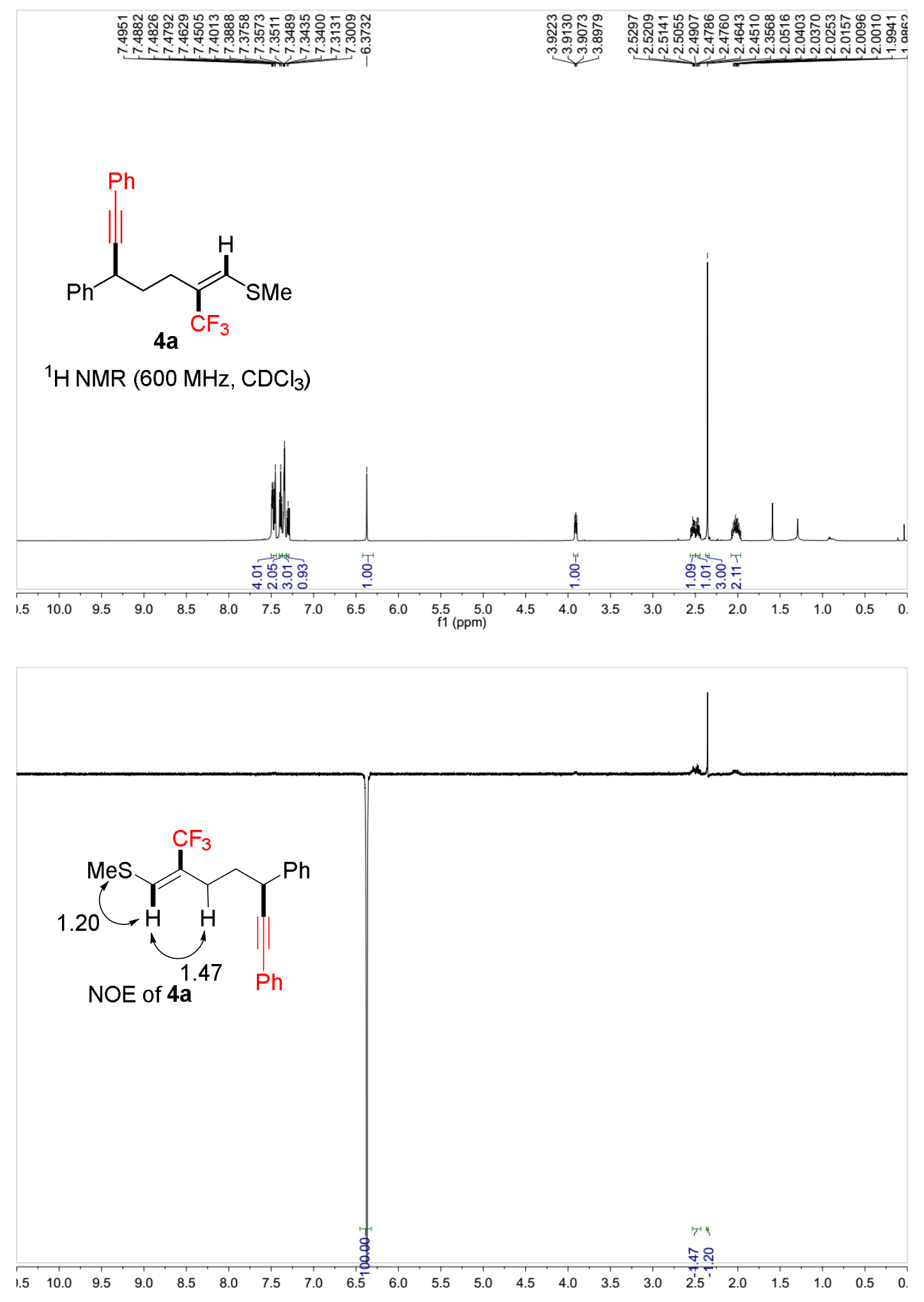

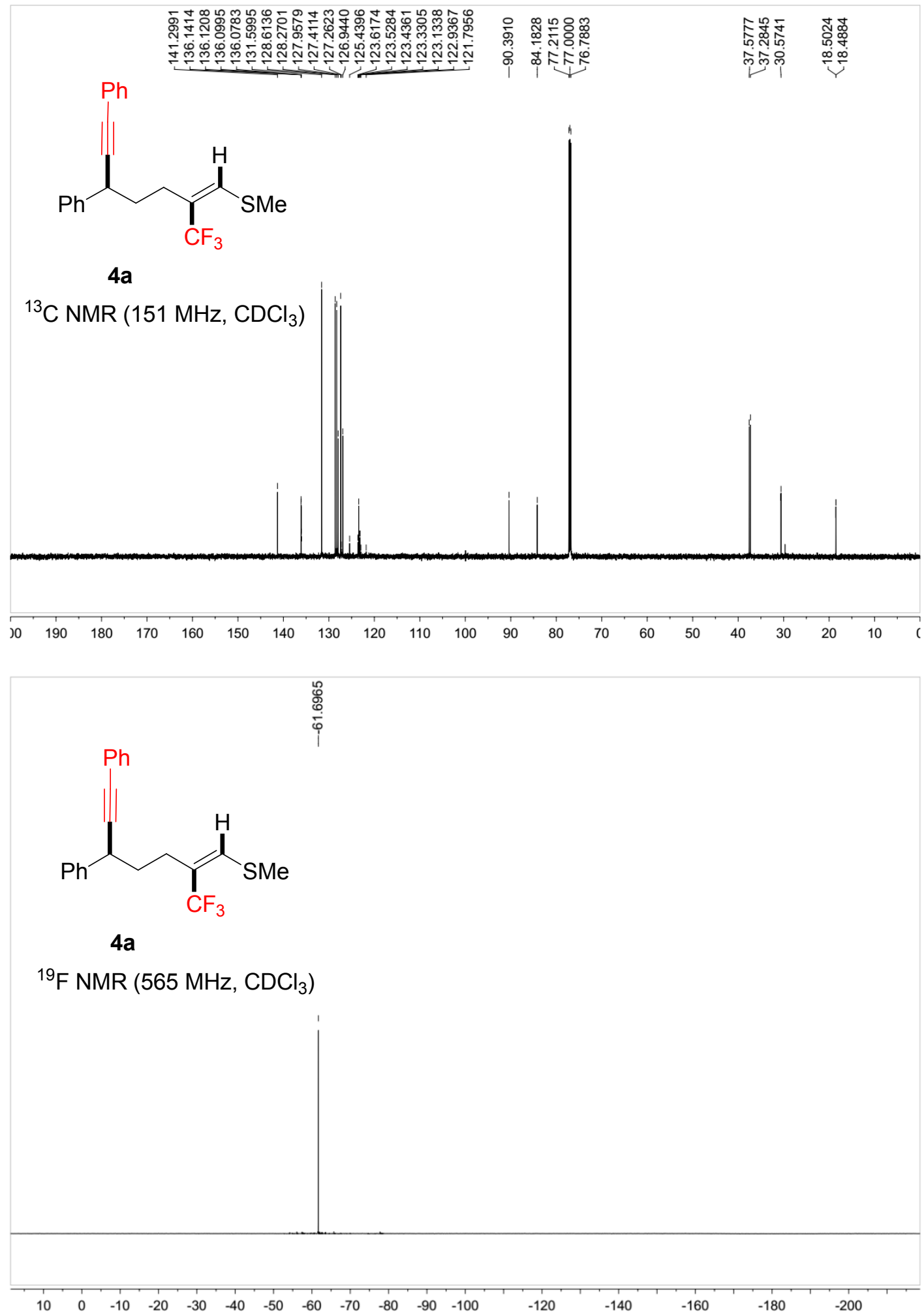


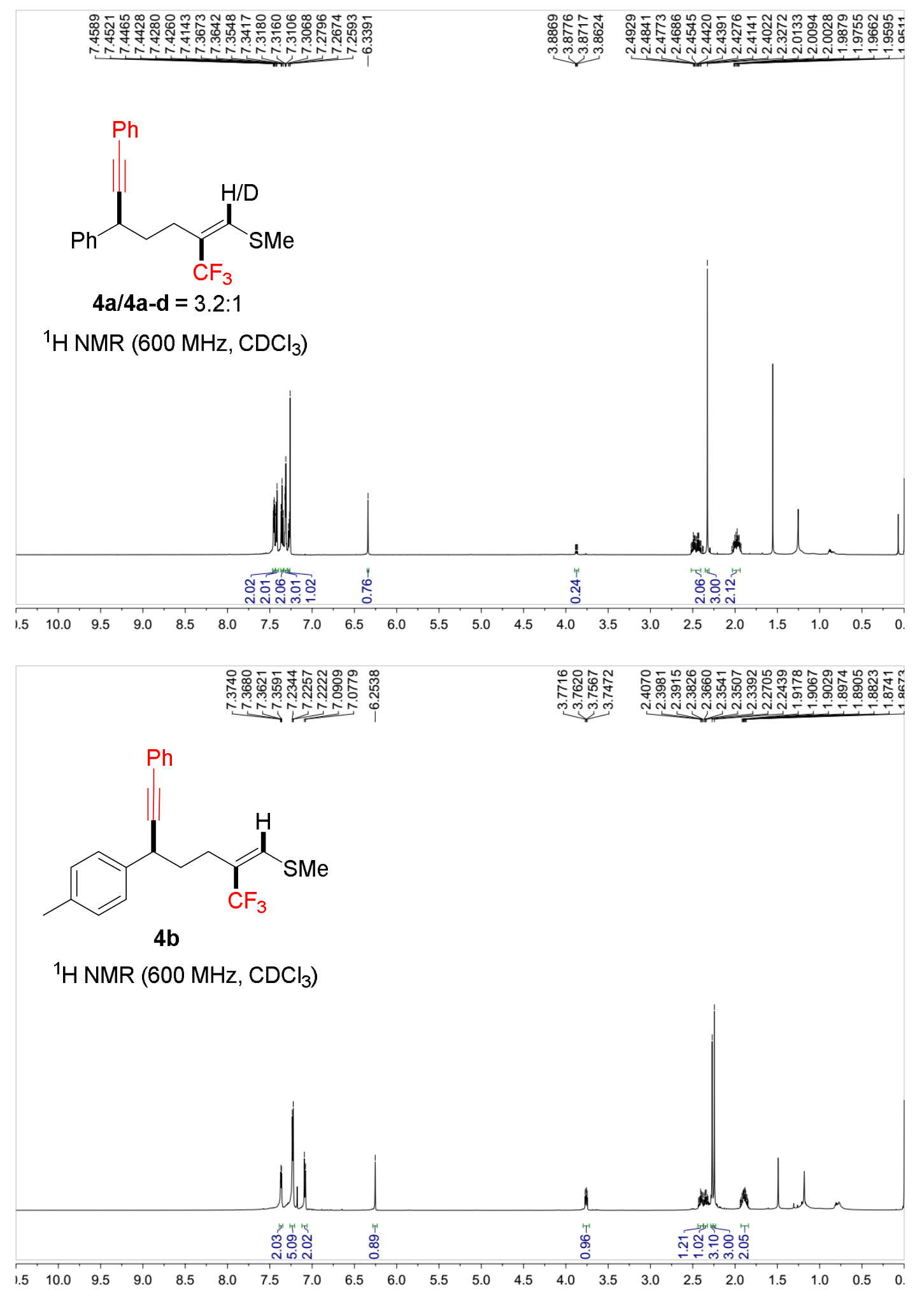




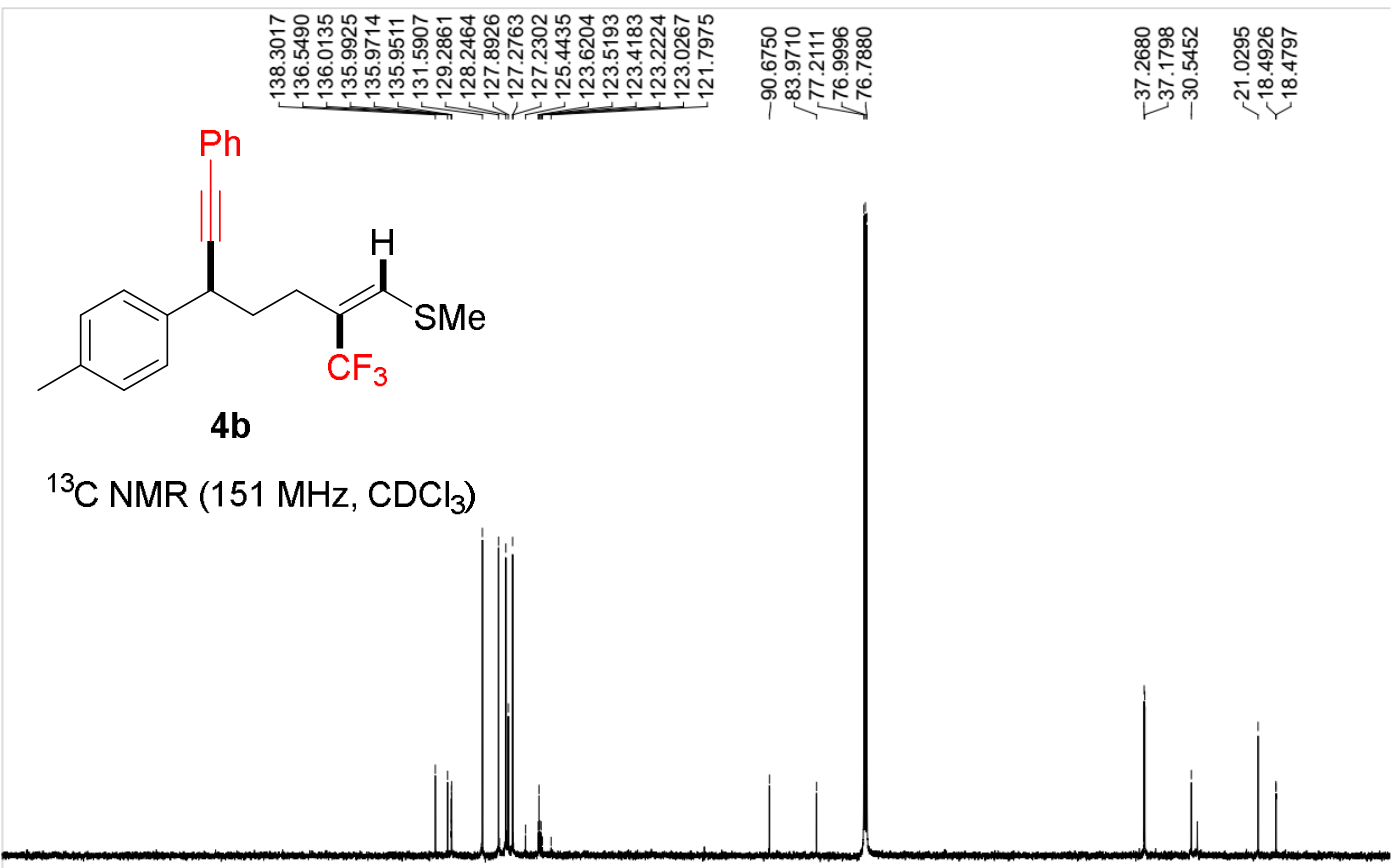

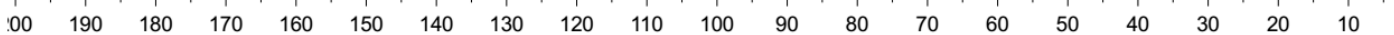<smiles>CO/C=C(\CCC(C#Cc1ccccc1)c1ccc(C)cc1)C(F)(F)F</smiles>

4b

${ }^{19} \mathrm{~F} \mathrm{NMR}\left(565 \mathrm{MHz}, \mathrm{CDCl}_{3}\right)$

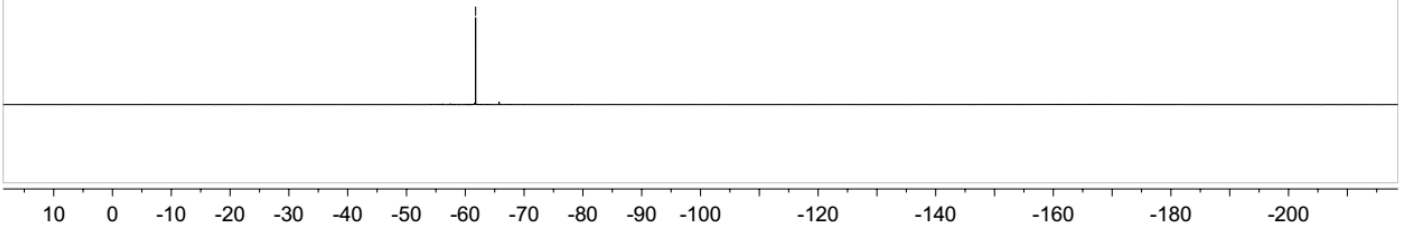



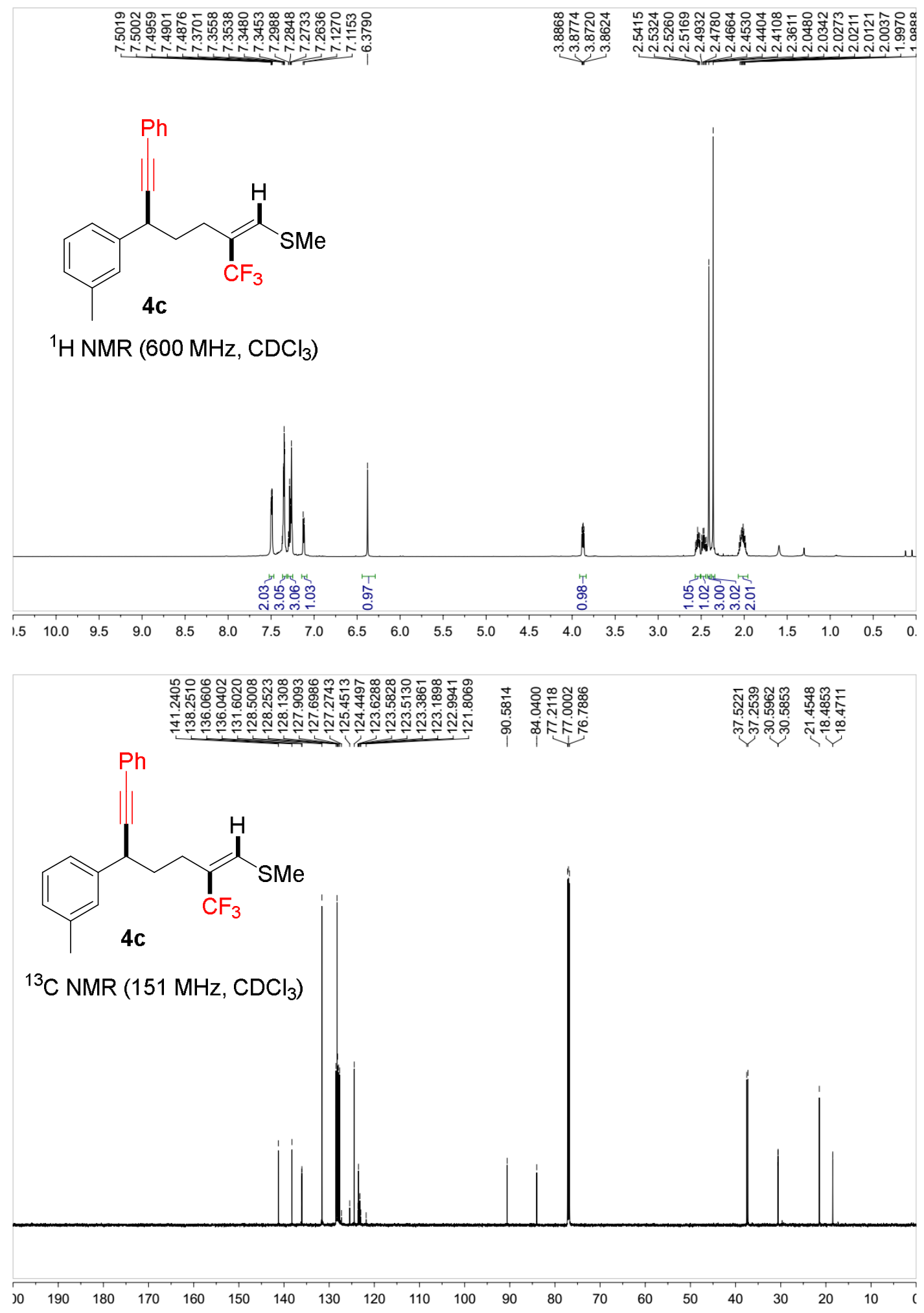

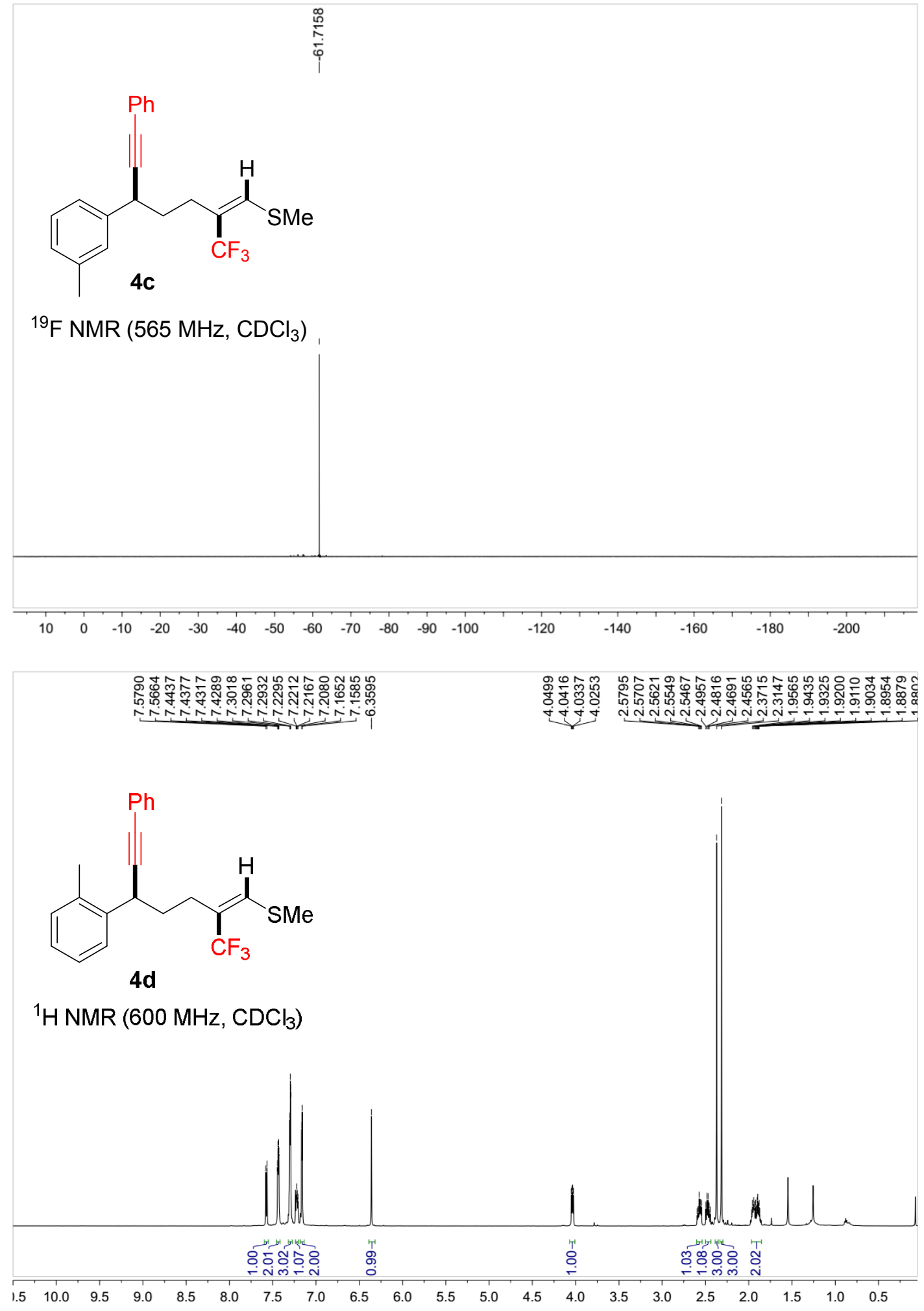

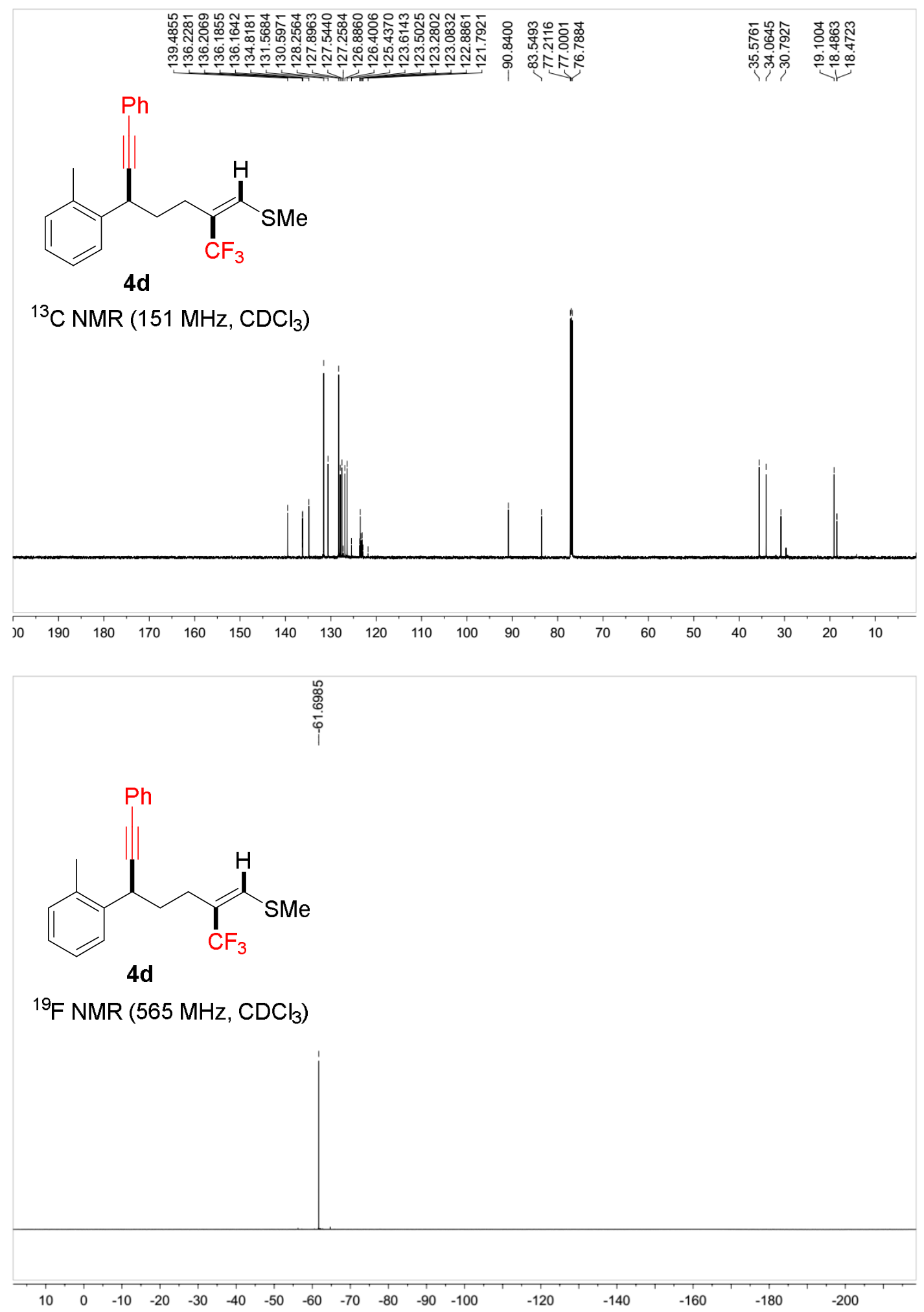


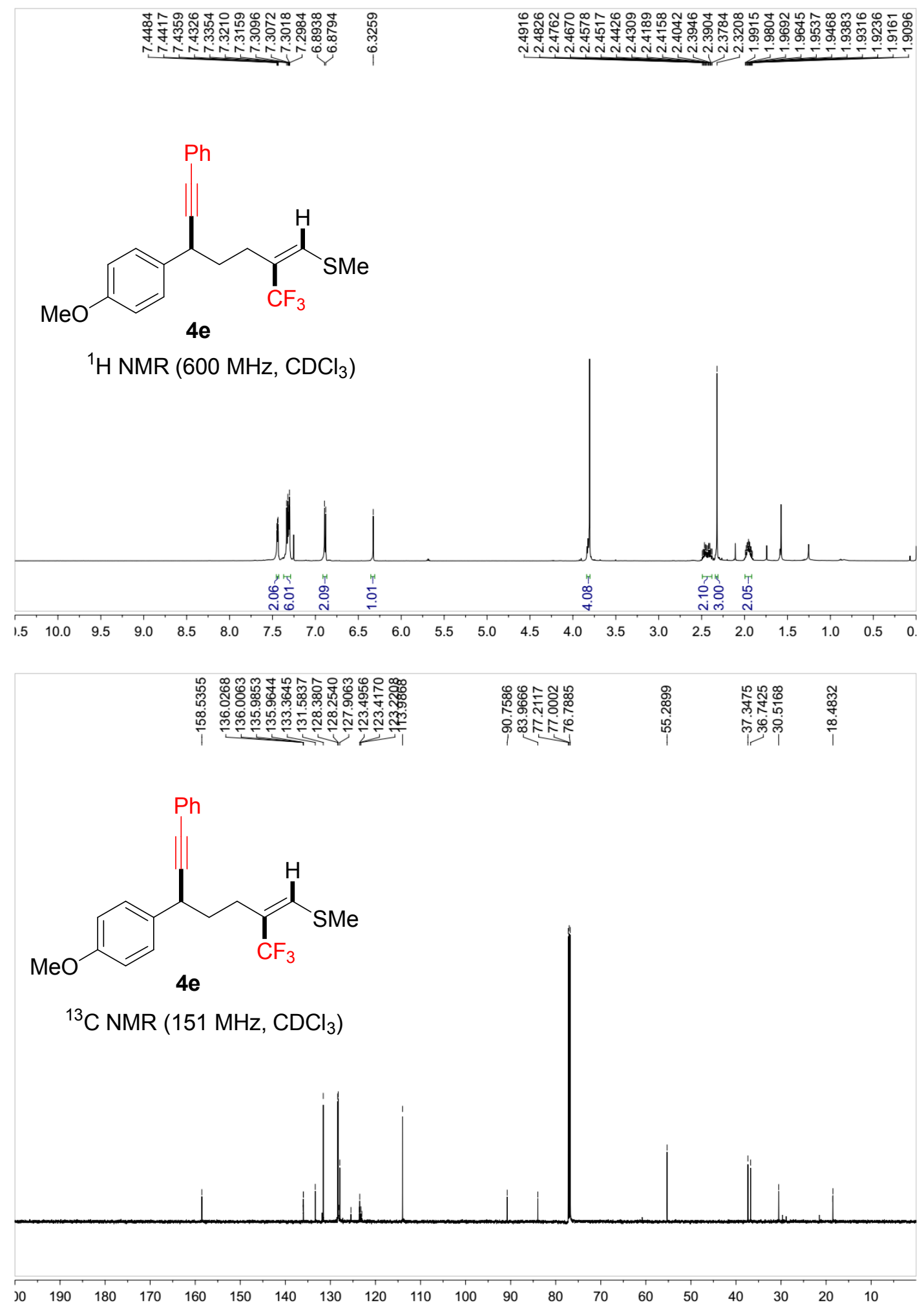



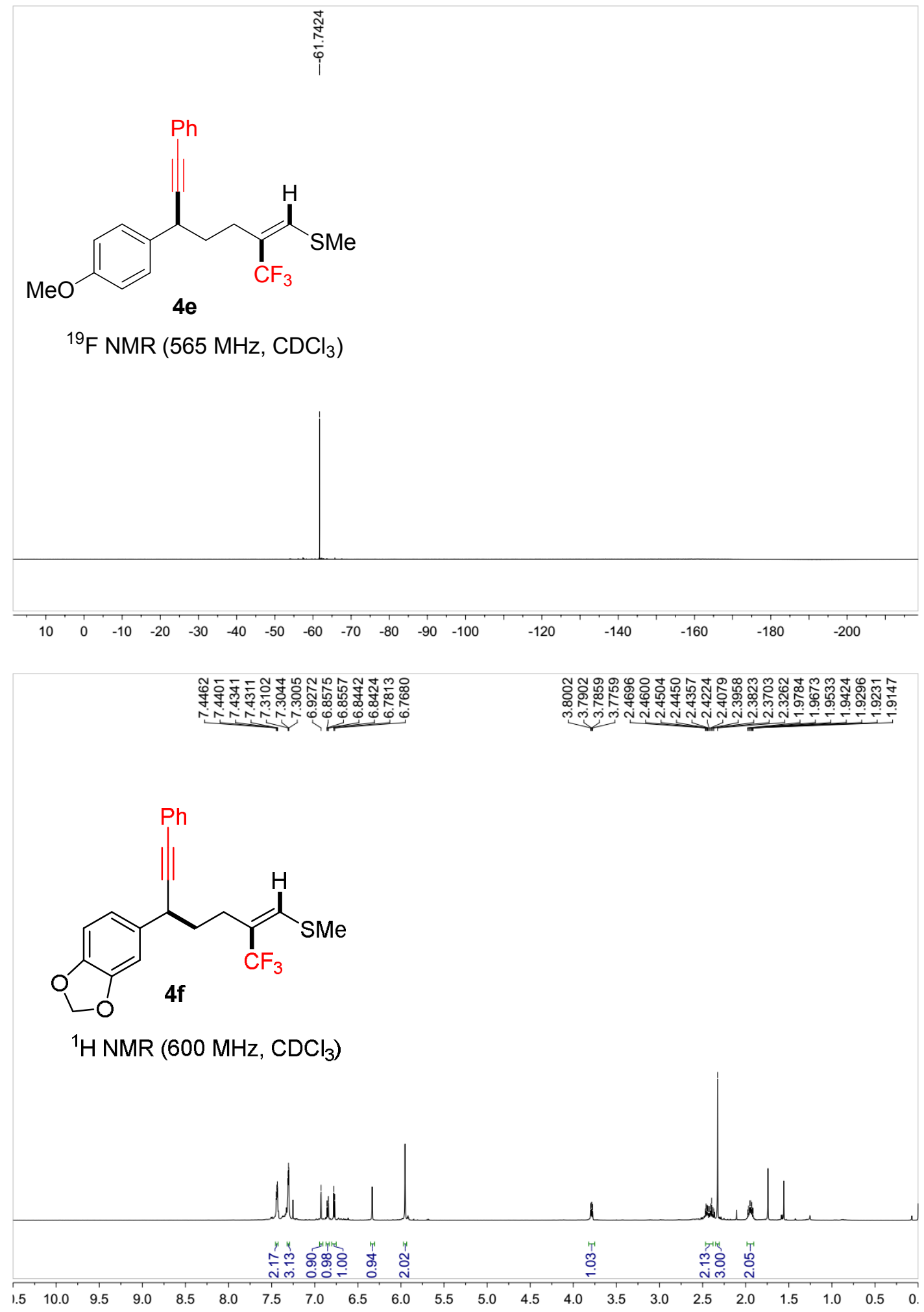

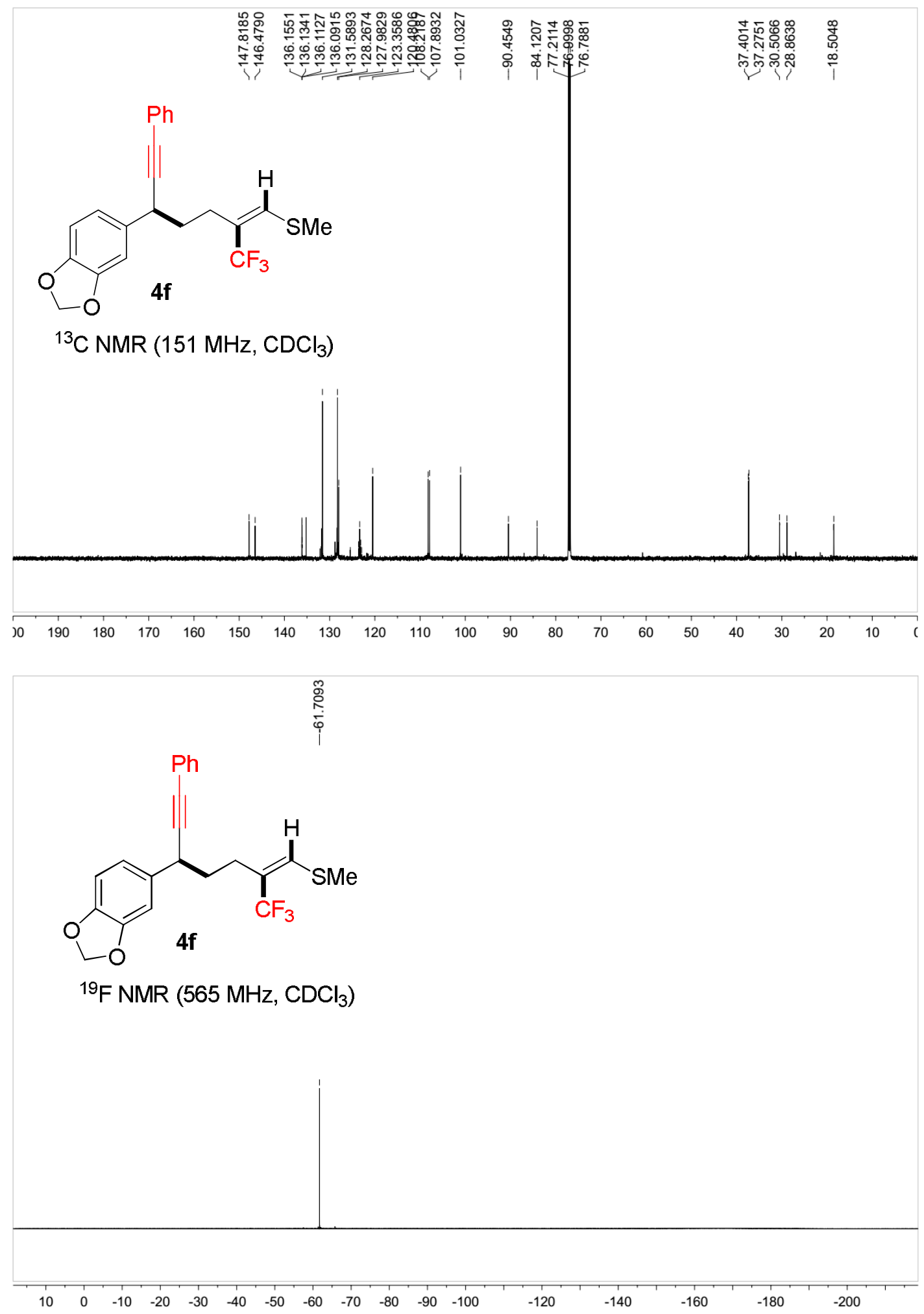

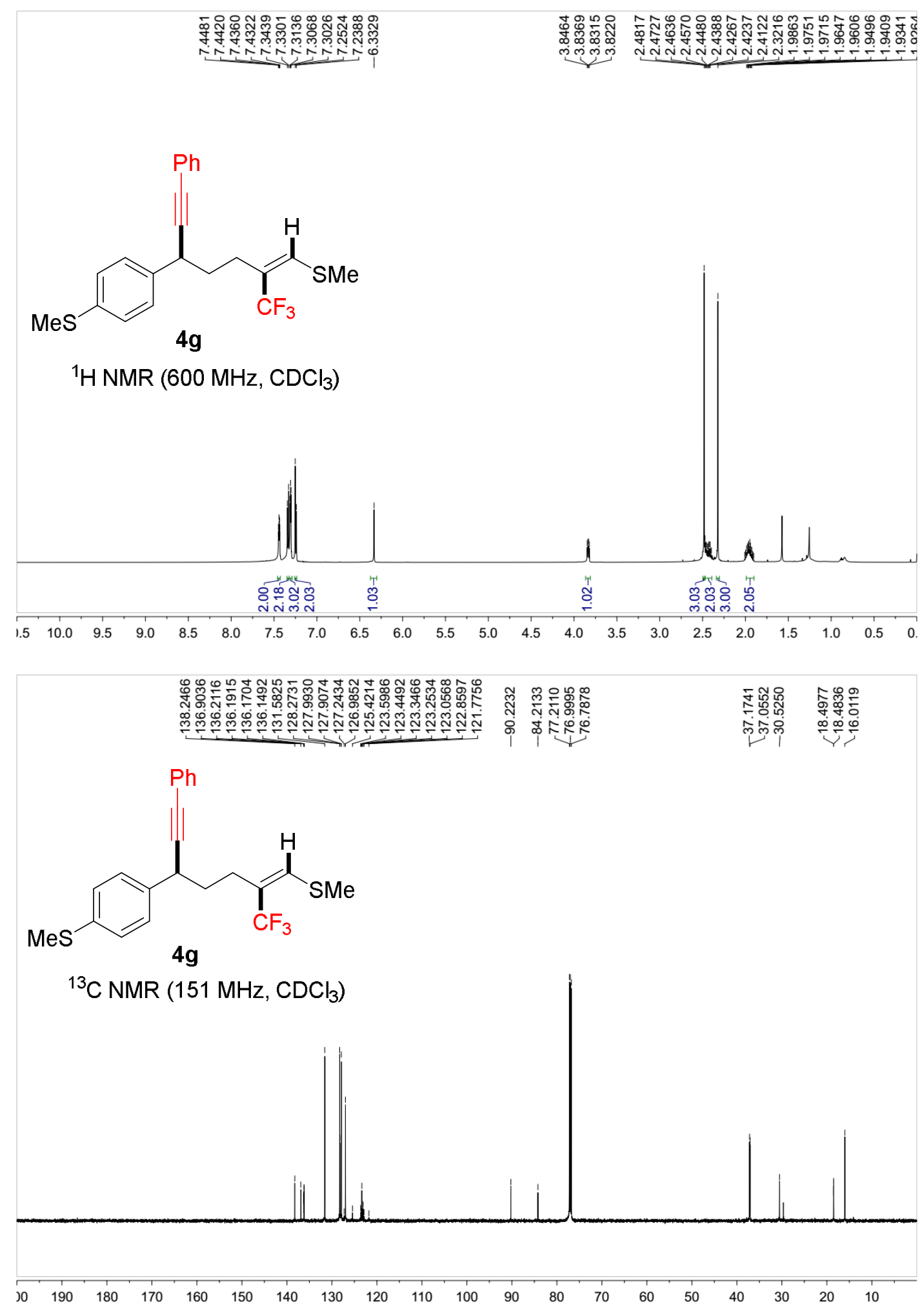


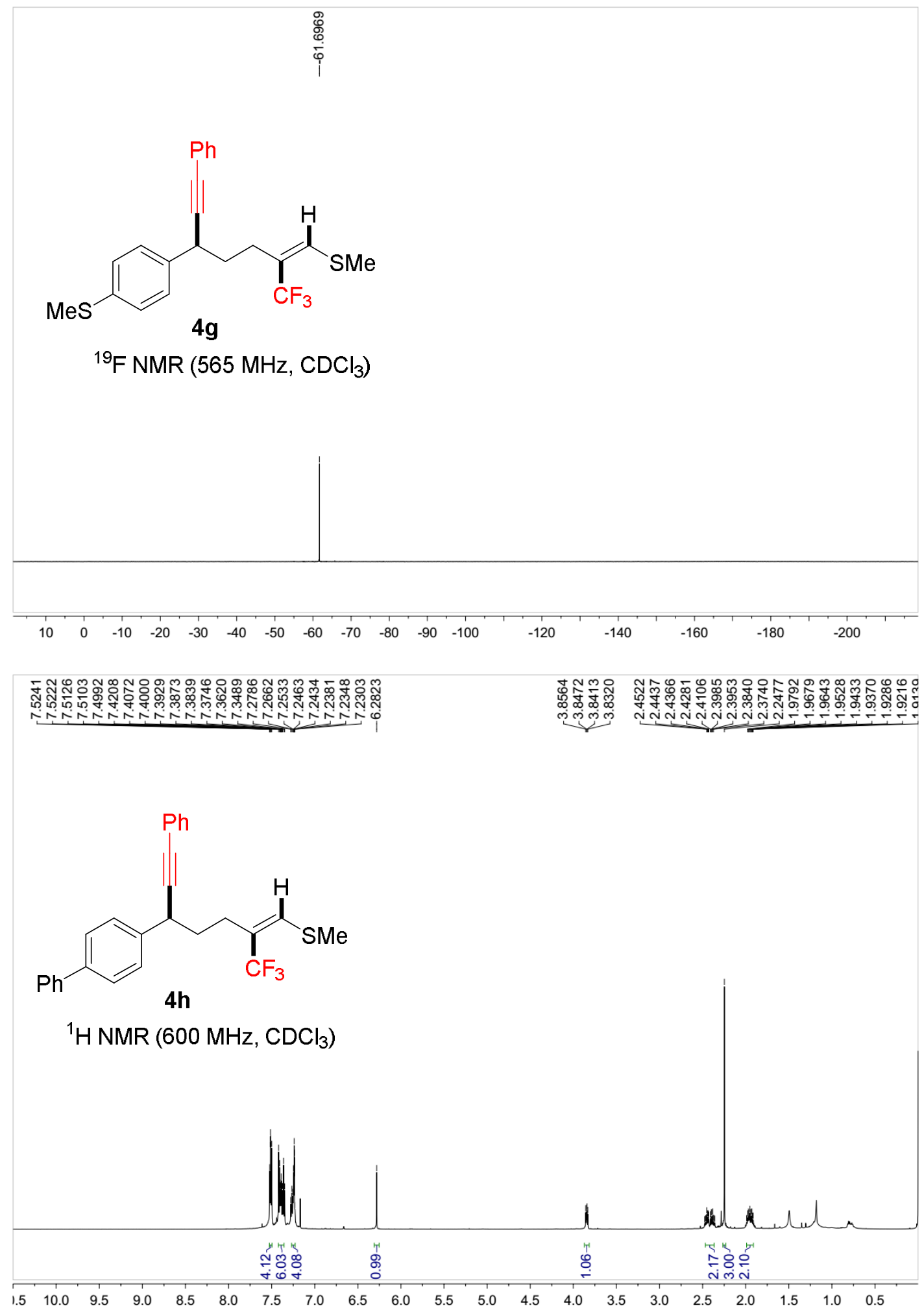




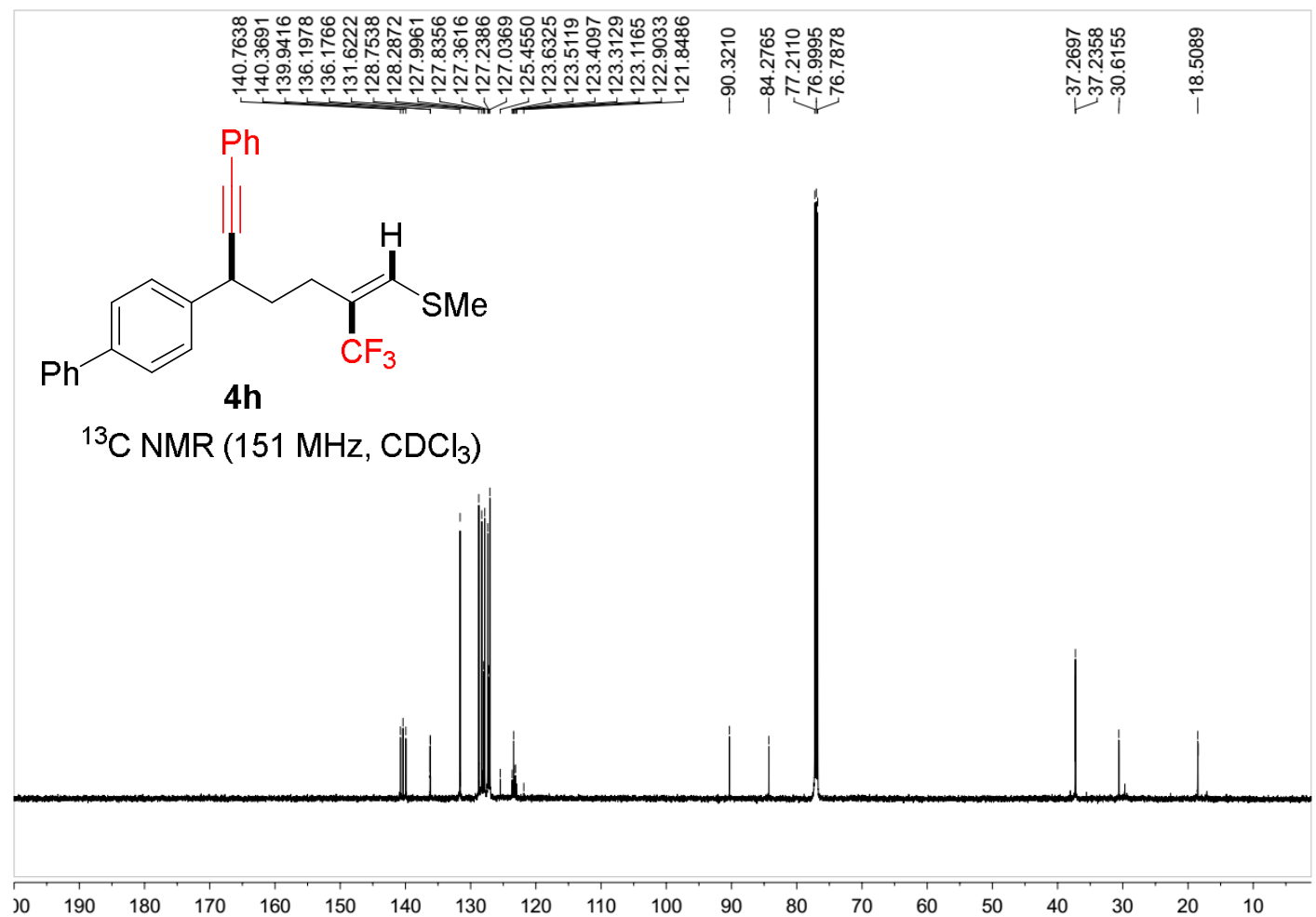

윰<smiles>CO/C=C(\CCC(C#Cc1ccccc1)c1ccc(-c2ccccc2)cc1)C(F)(F)F</smiles>

${ }^{19} \mathrm{~F} \mathrm{NMR}\left(565 \mathrm{MHz}, \mathrm{CDCl}_{3}\right)$

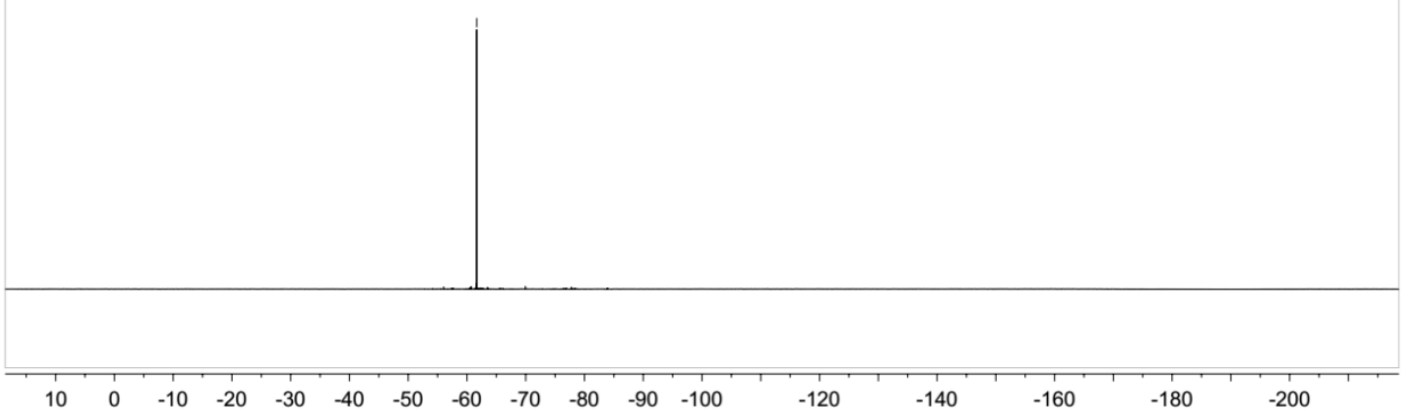




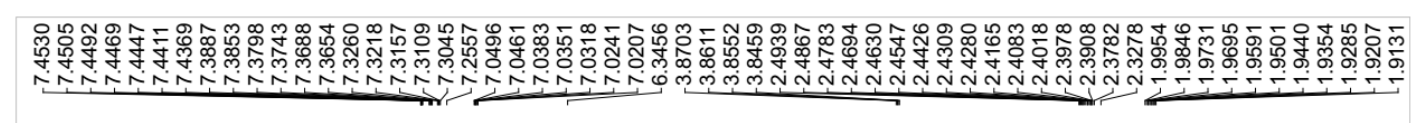<smiles>CO/C=C(\CCC(C#Cc1ccccc1)c1ccc(F)cc1)C(F)(F)F</smiles>

${ }^{1} \mathrm{H} \mathrm{NMR}\left(600 \mathrm{MHz}, \mathrm{CDCl}_{3}\right)$
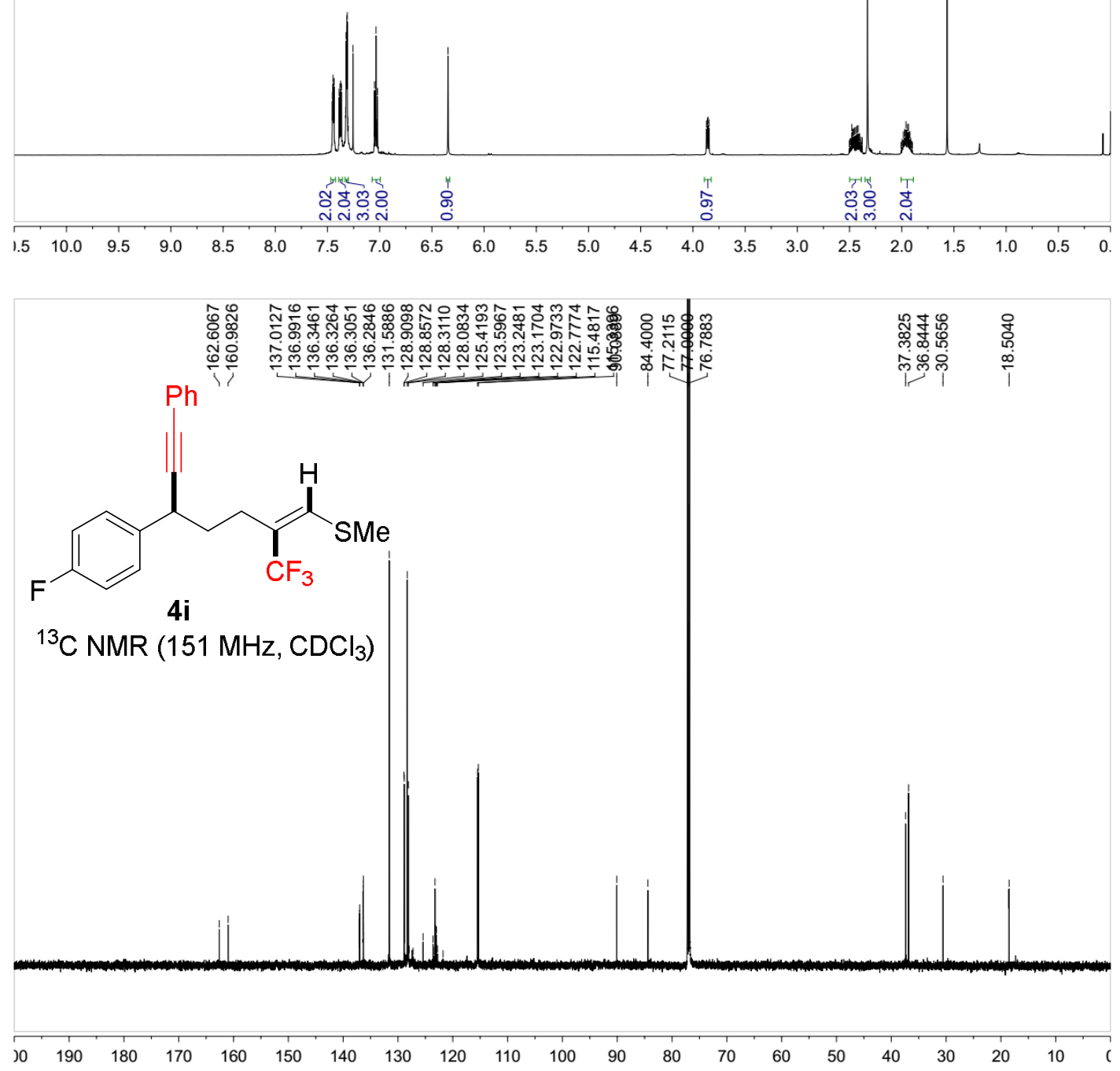

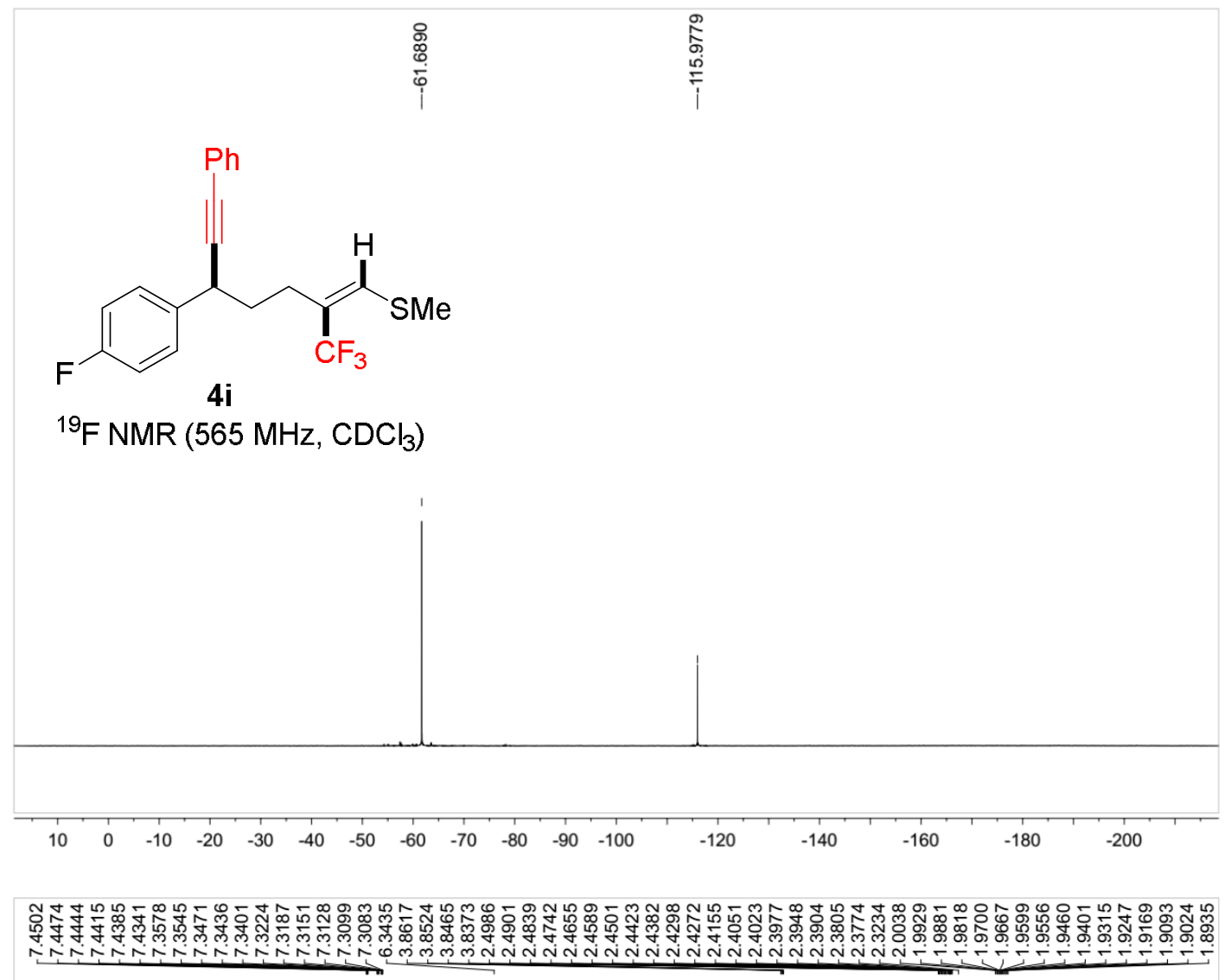<smiles>COC=C(CCC(C#Cc1ccccc1)c1ccc(Cl)cc1)C(F)(F)F</smiles>

${ }^{1} \mathrm{H}$ NMR $\left(600 \mathrm{MHz}, \mathrm{CDCl}_{3}\right)$

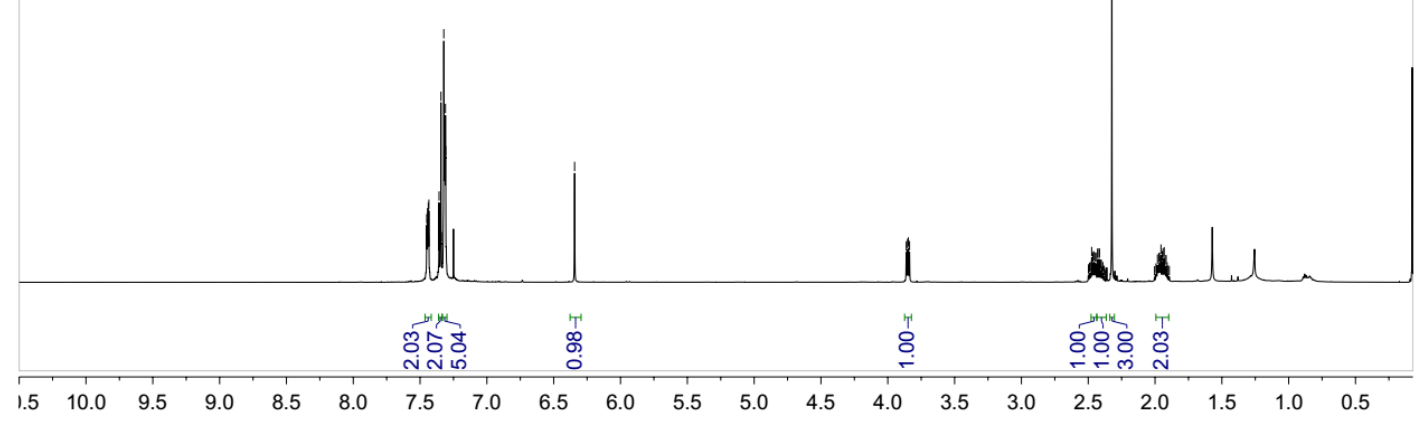



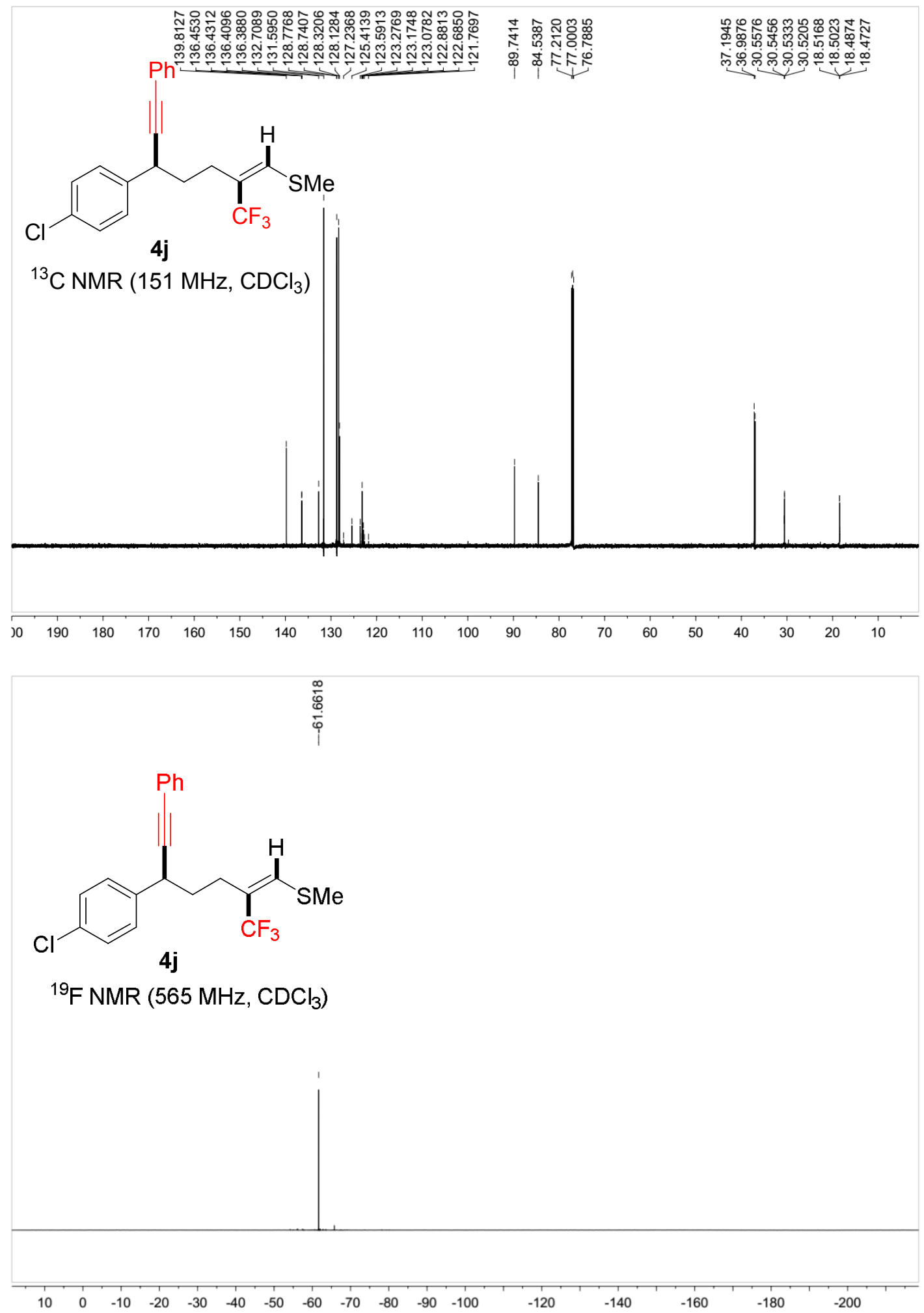

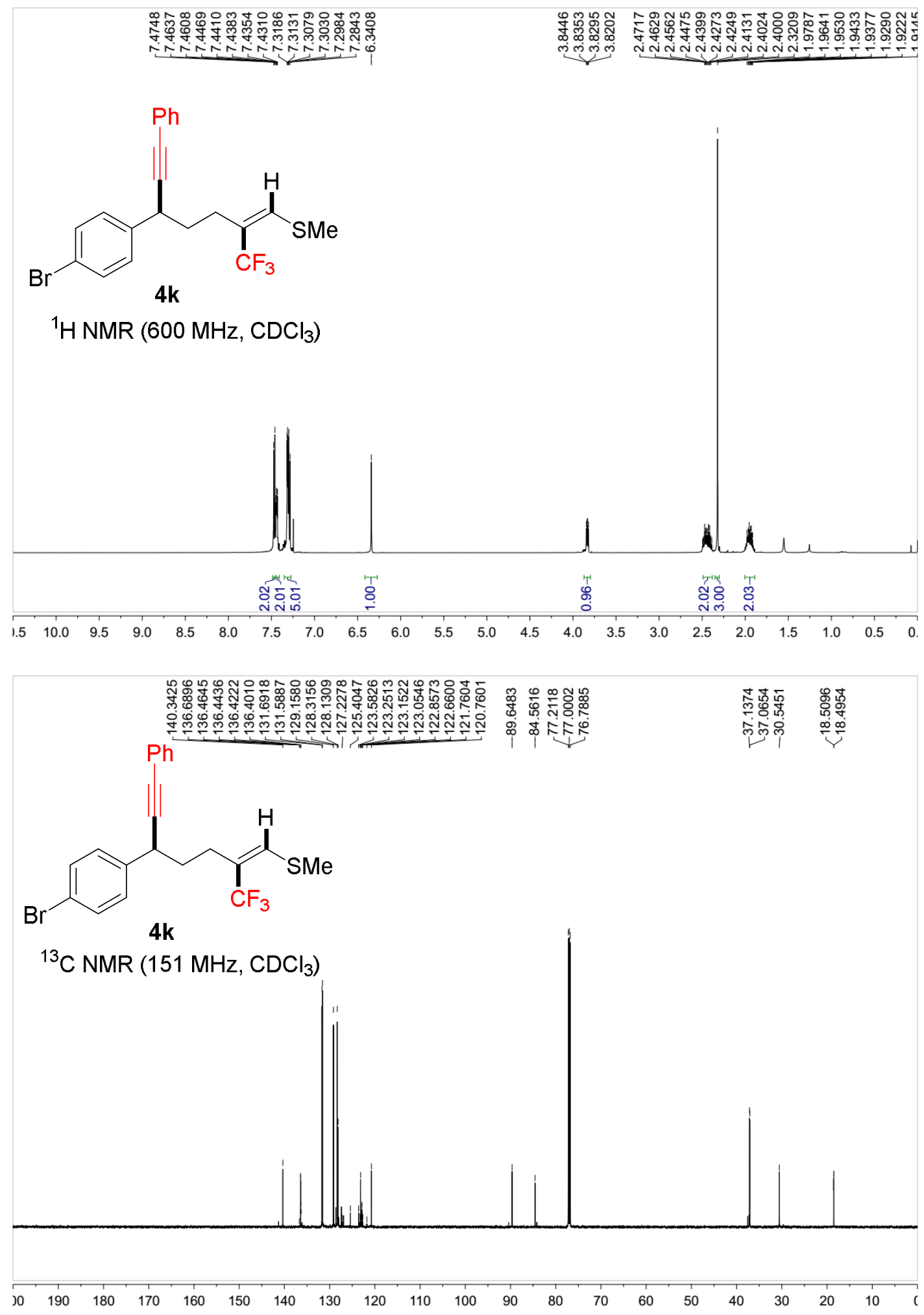


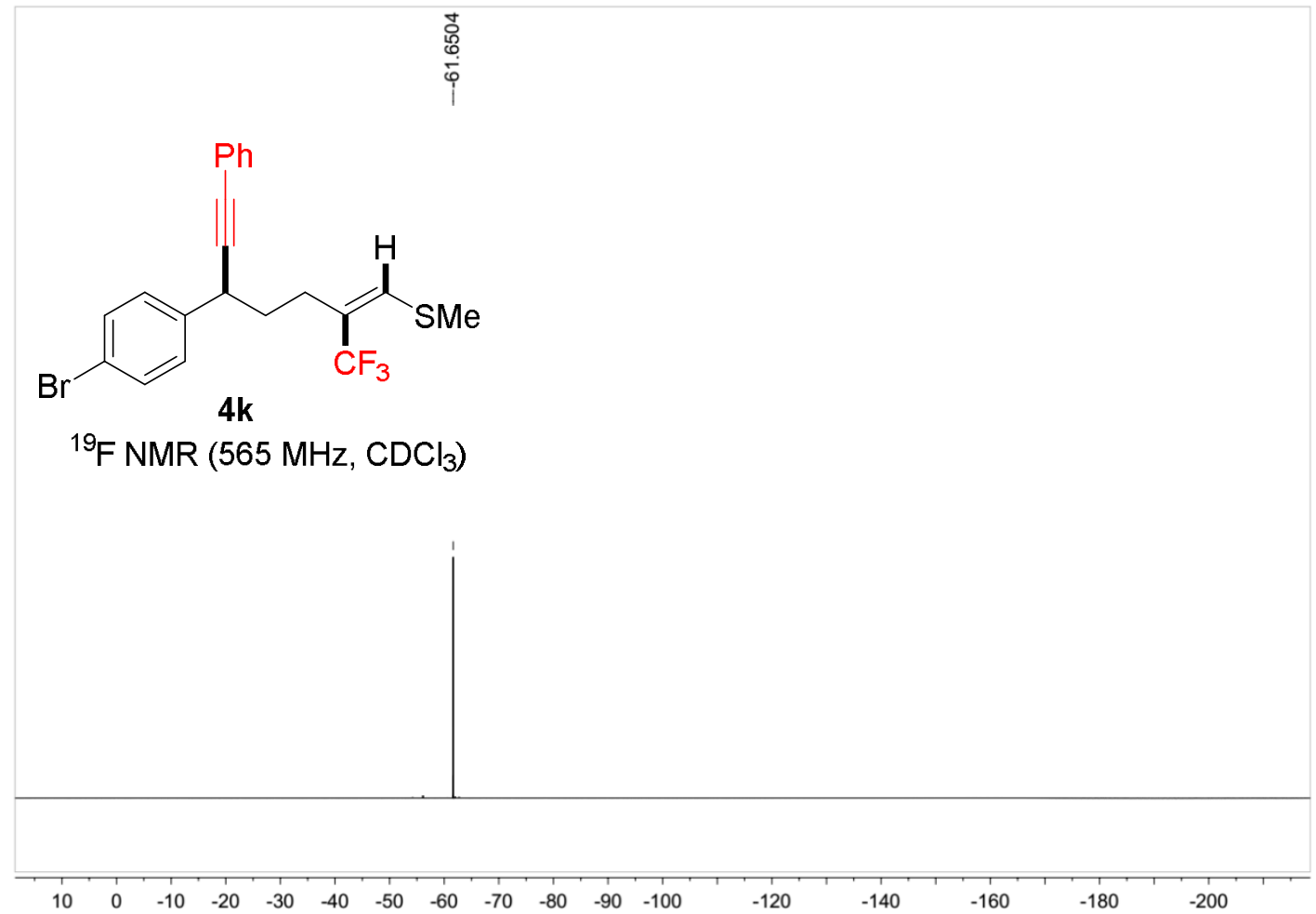

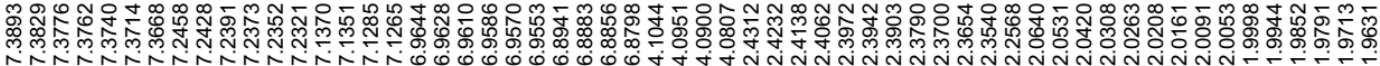

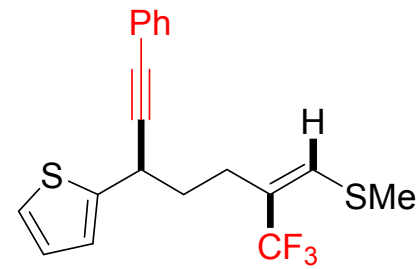

41

${ }^{1} \mathrm{H}$ NMR $\left(600 \mathrm{MHz}, \mathrm{CDCl}_{3}\right)$

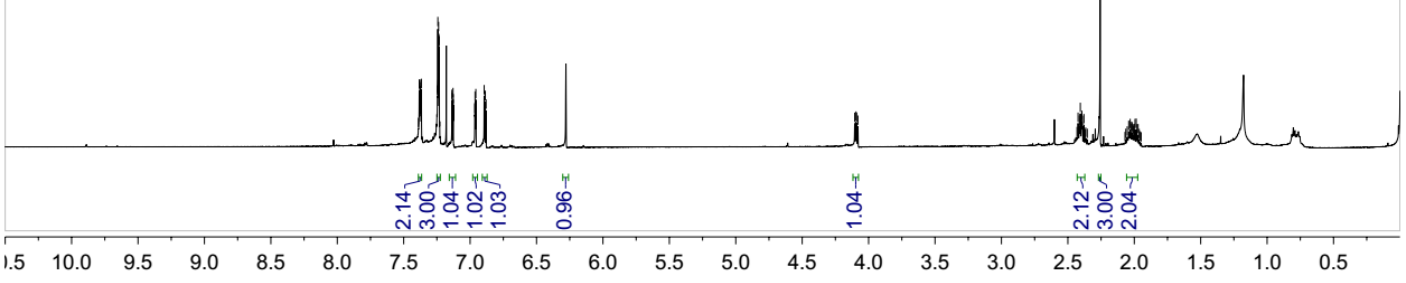



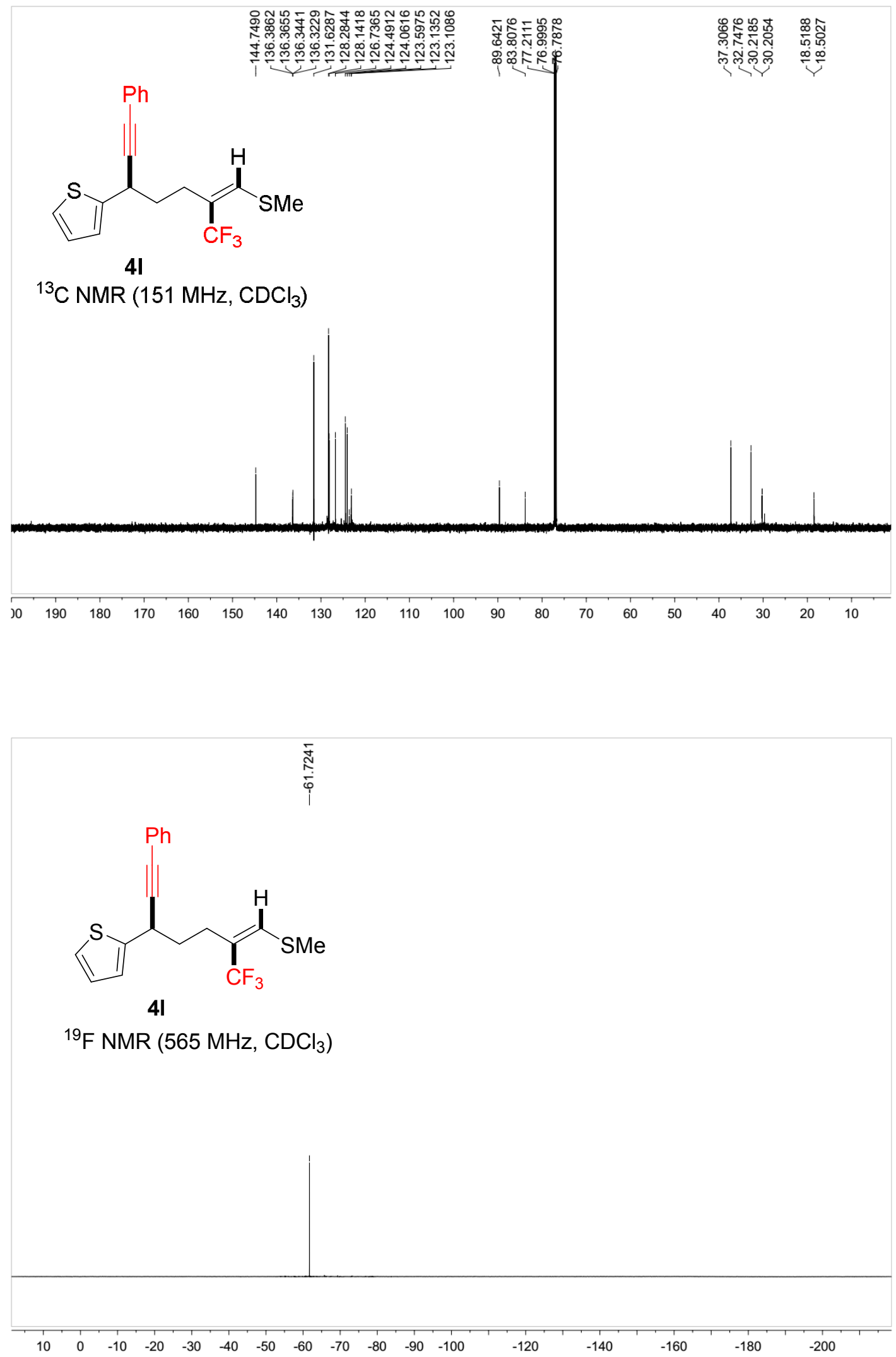

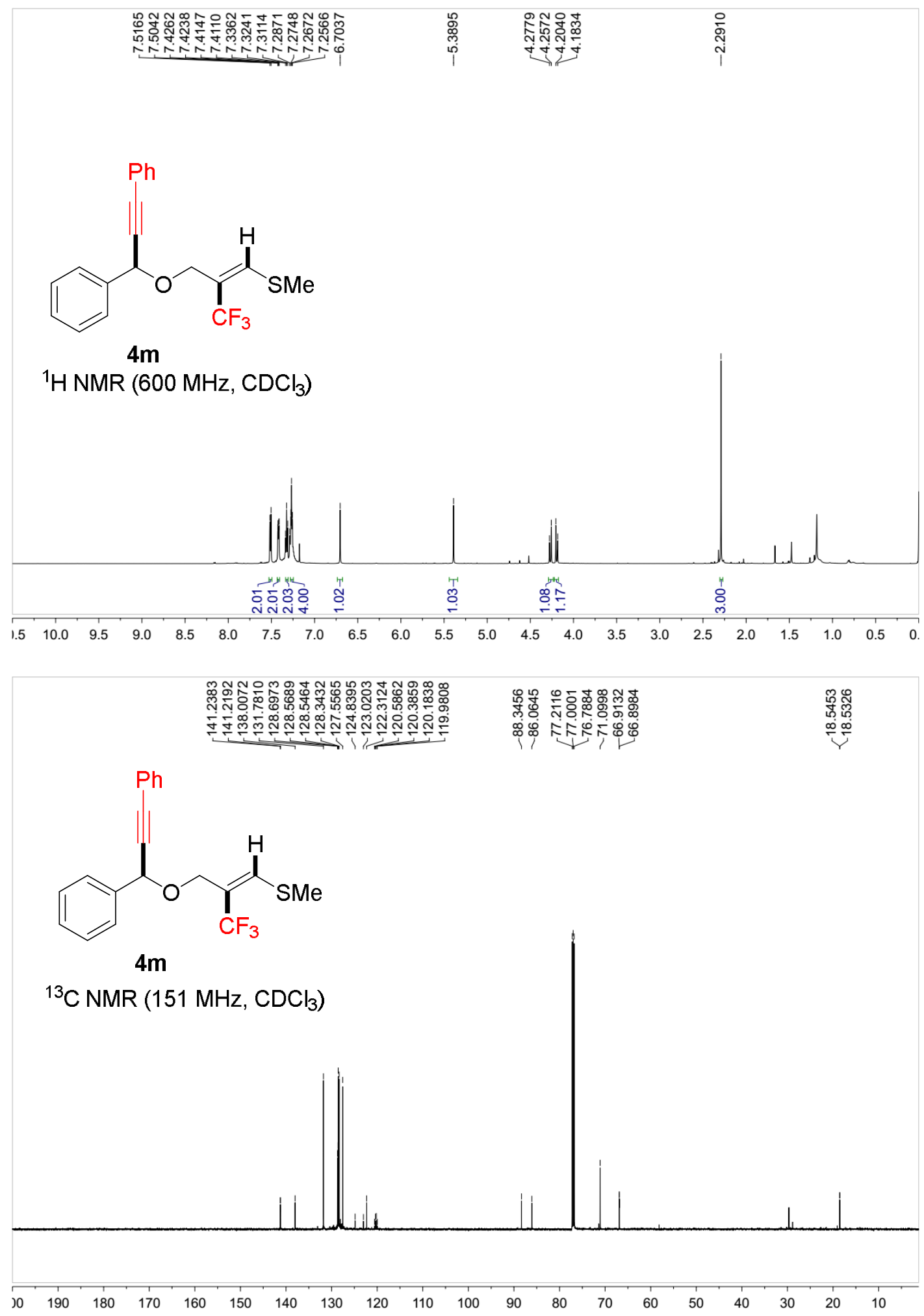

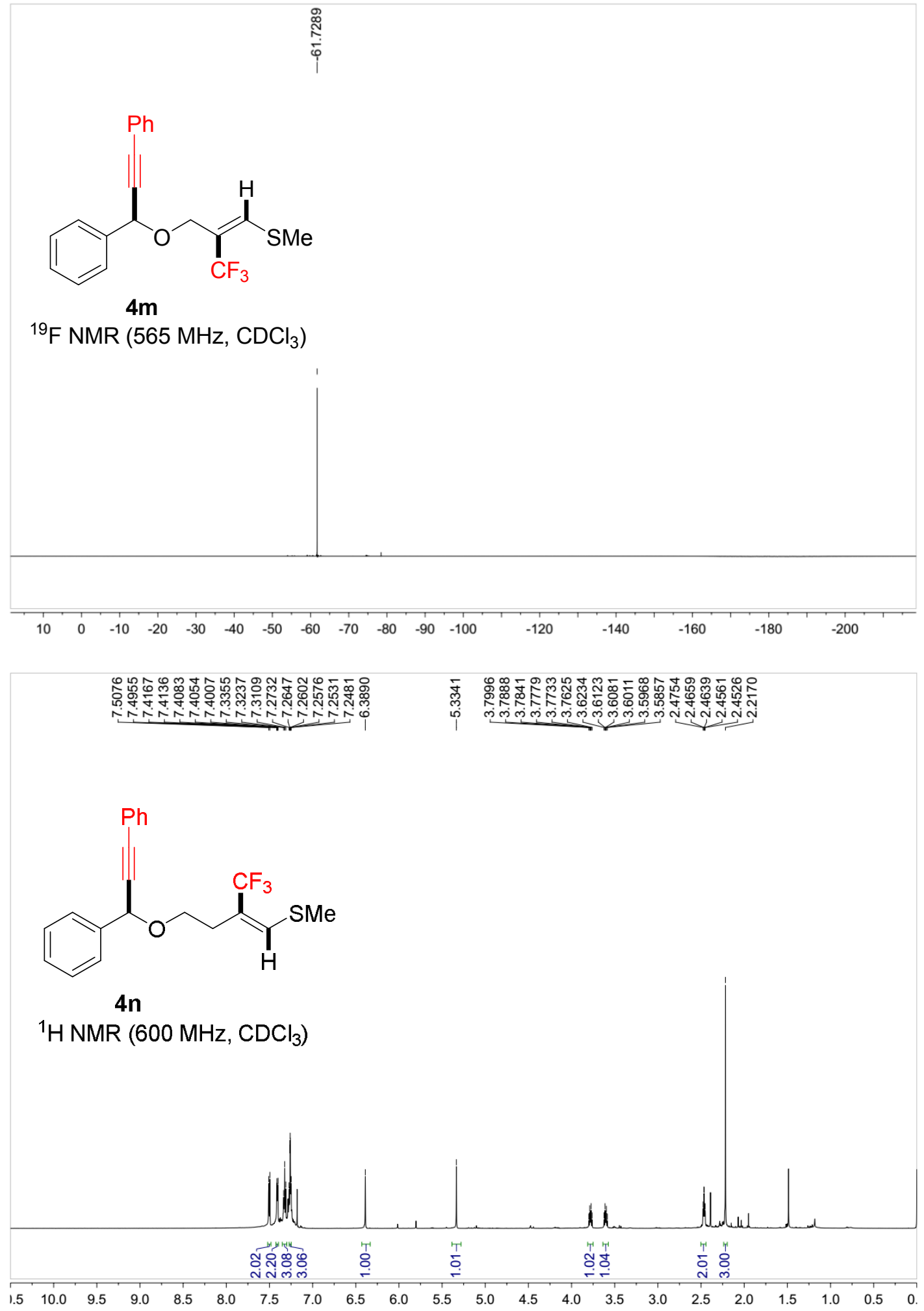


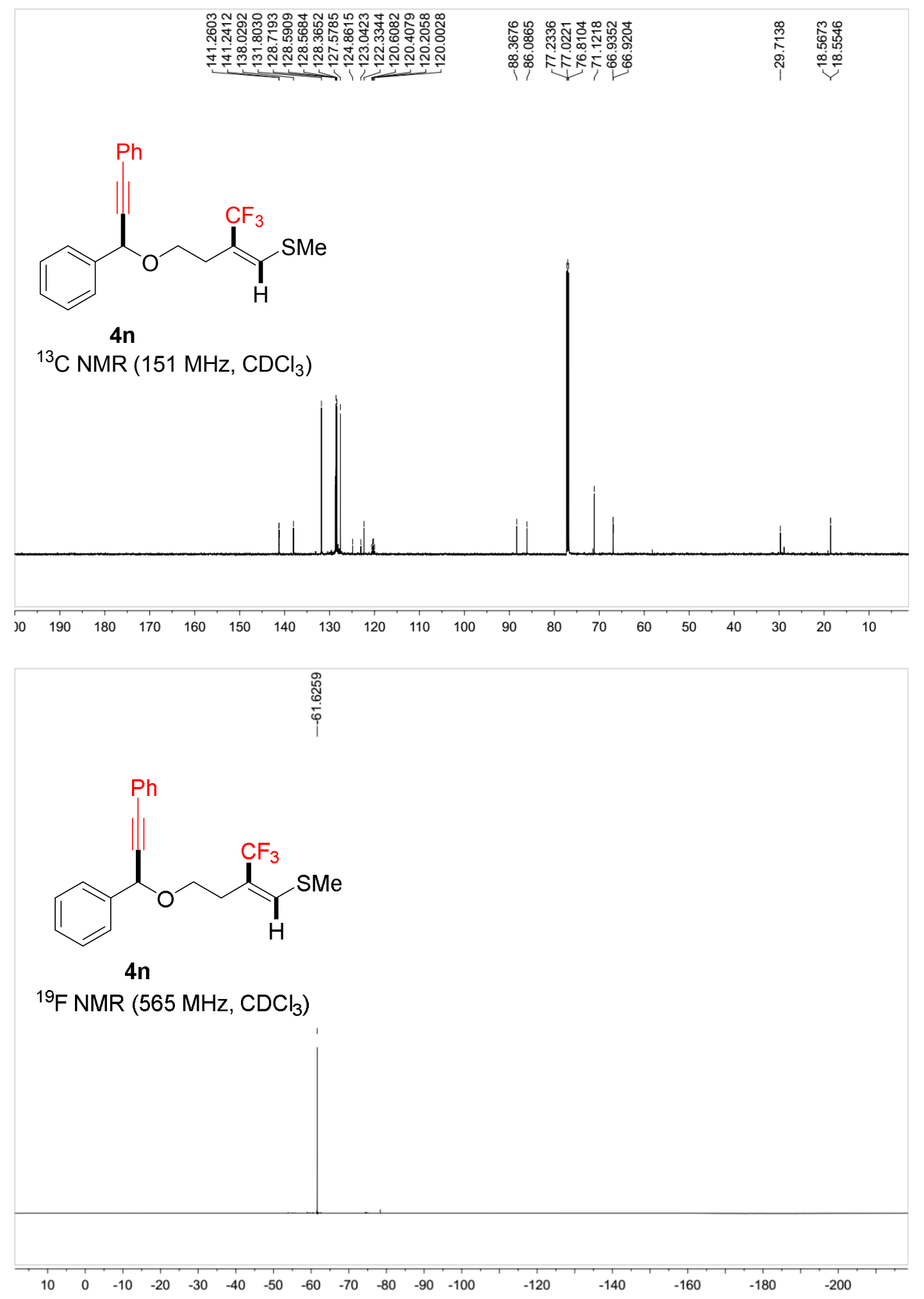



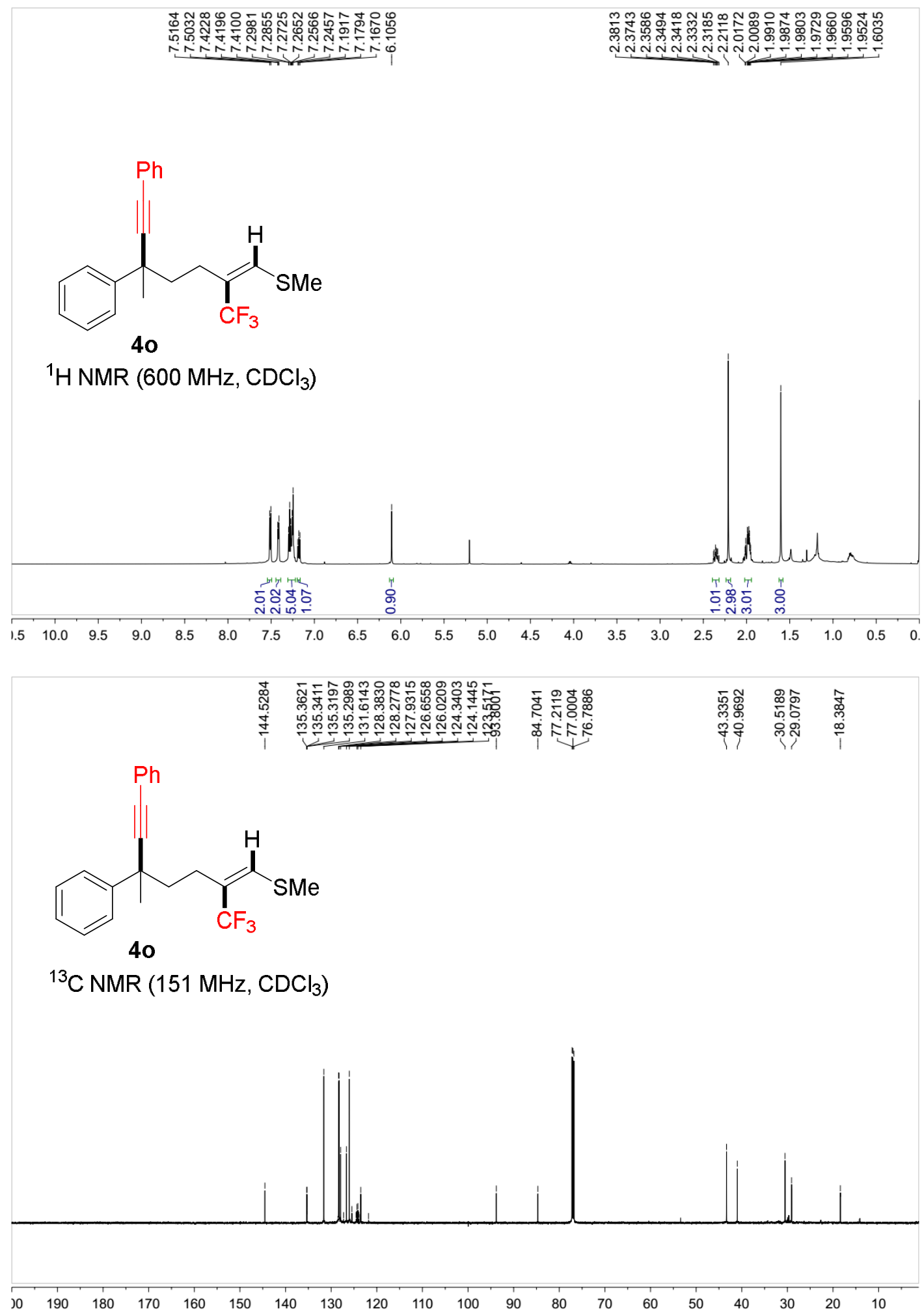


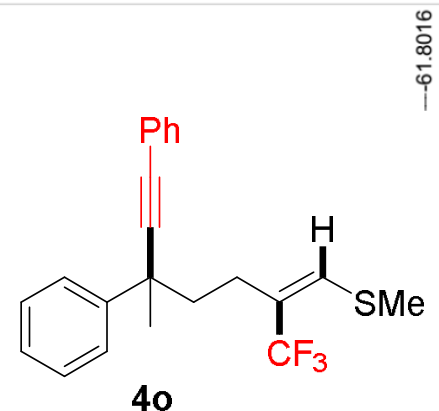

${ }^{19} \mathrm{~F} \mathrm{NMR}\left(565 \mathrm{MHz}, \mathrm{CDCl}_{3}\right)$
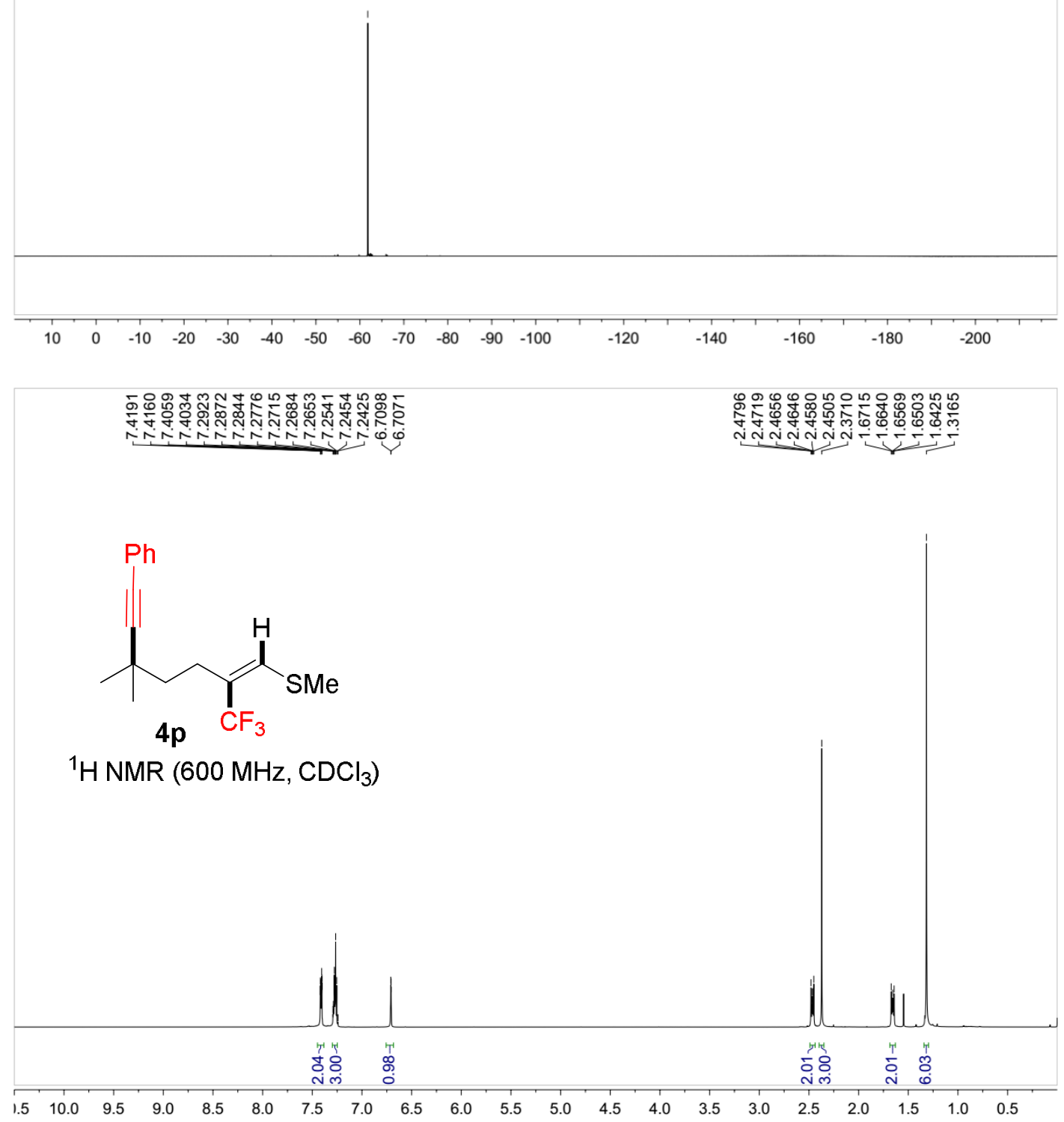


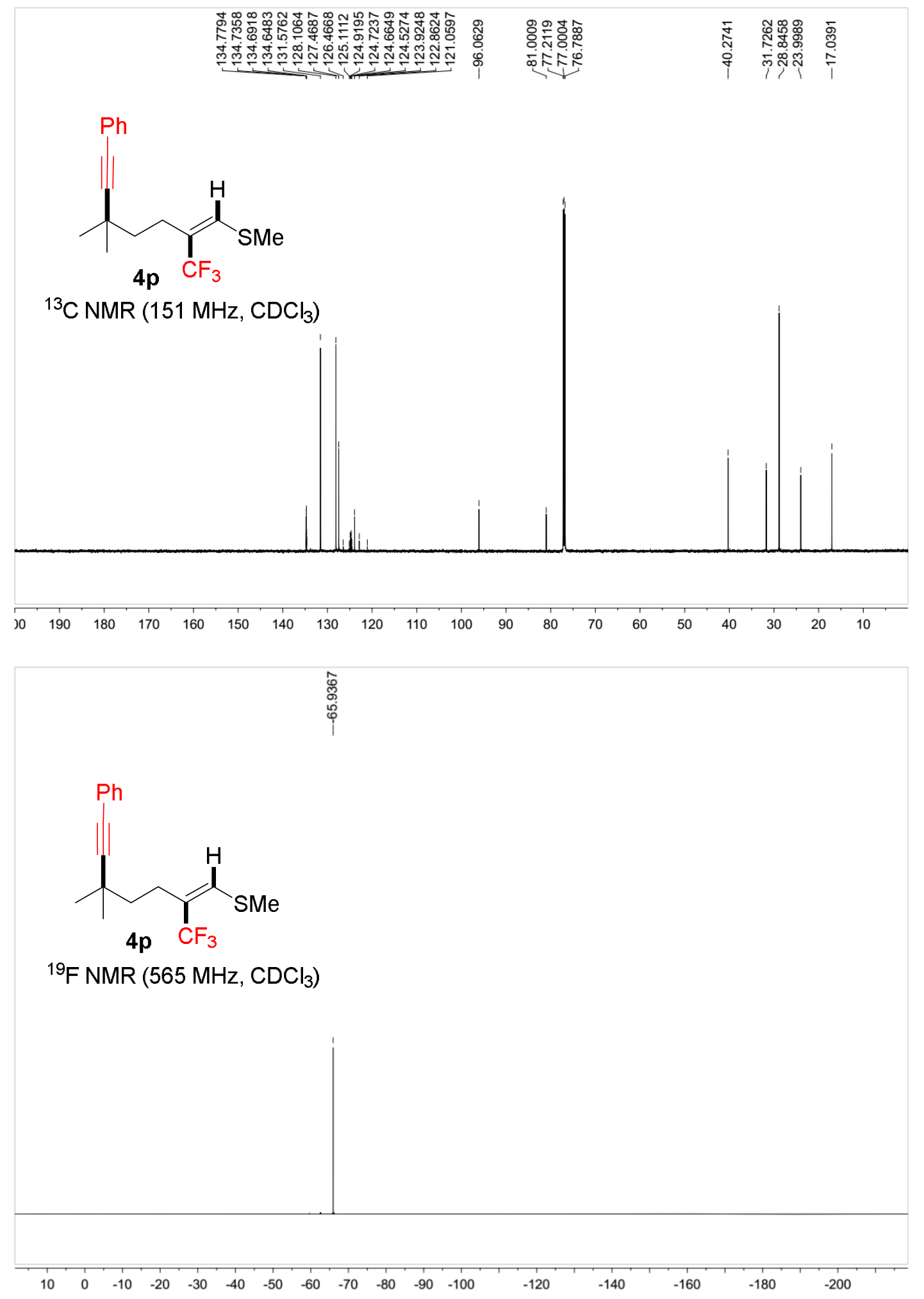




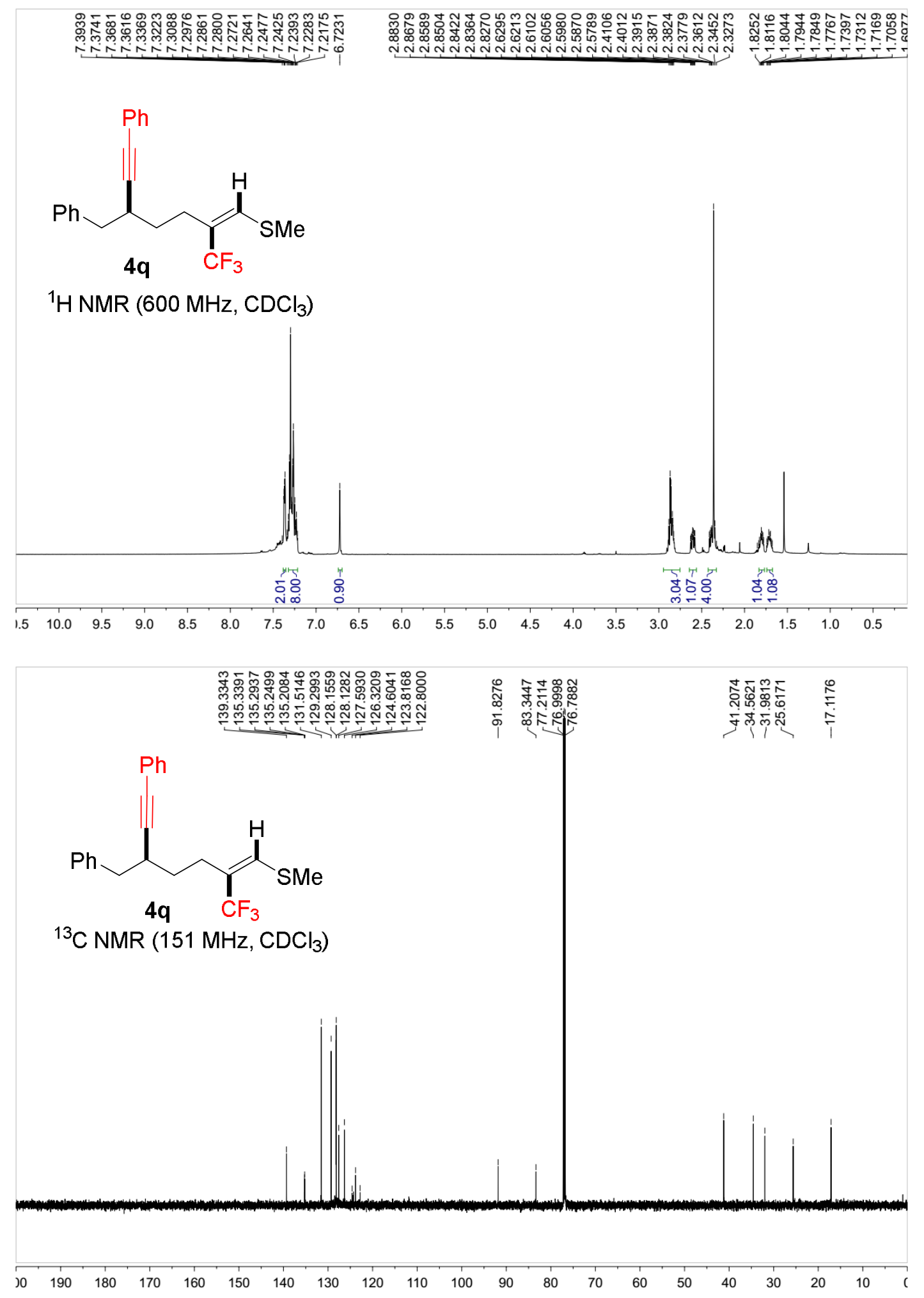



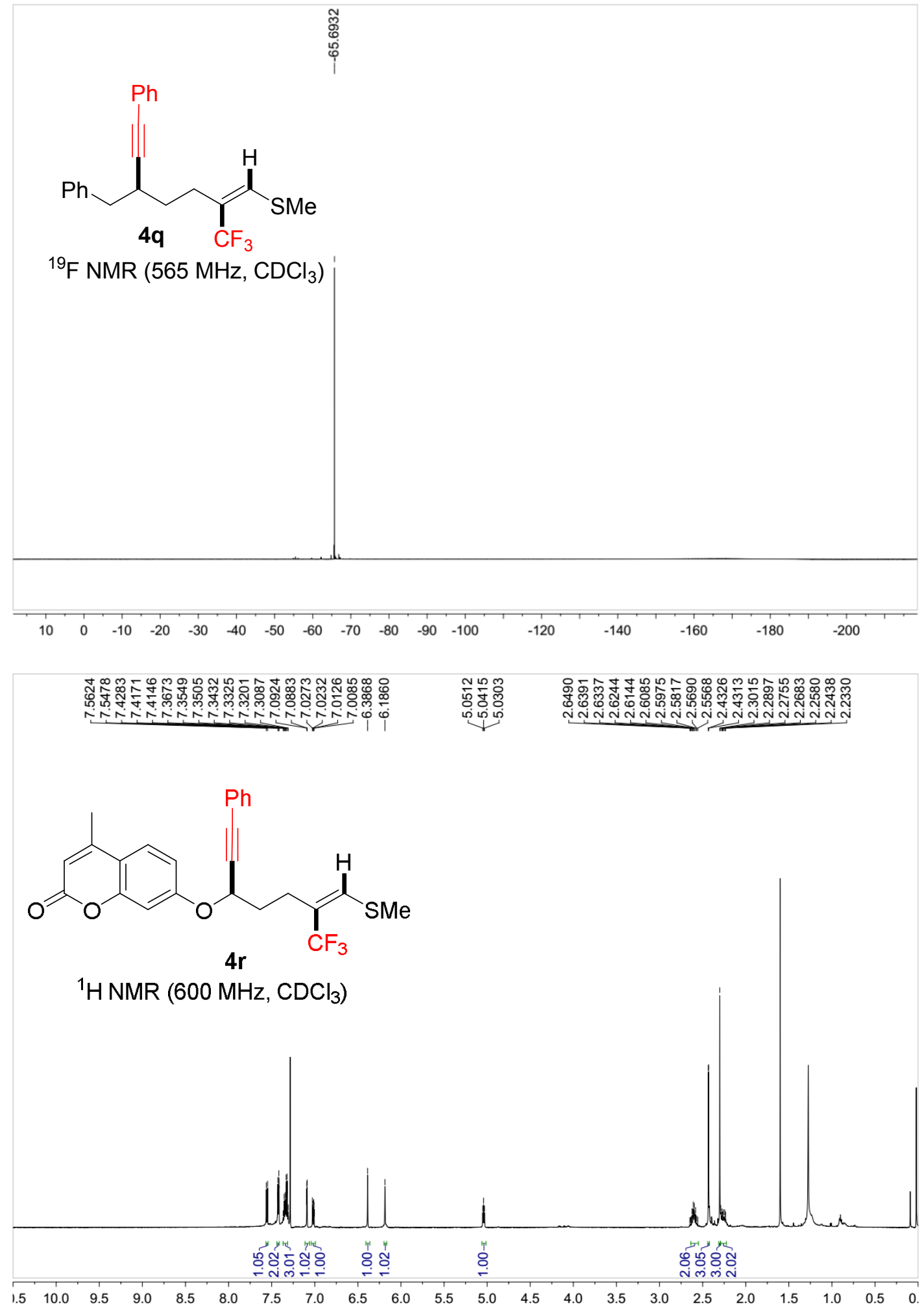

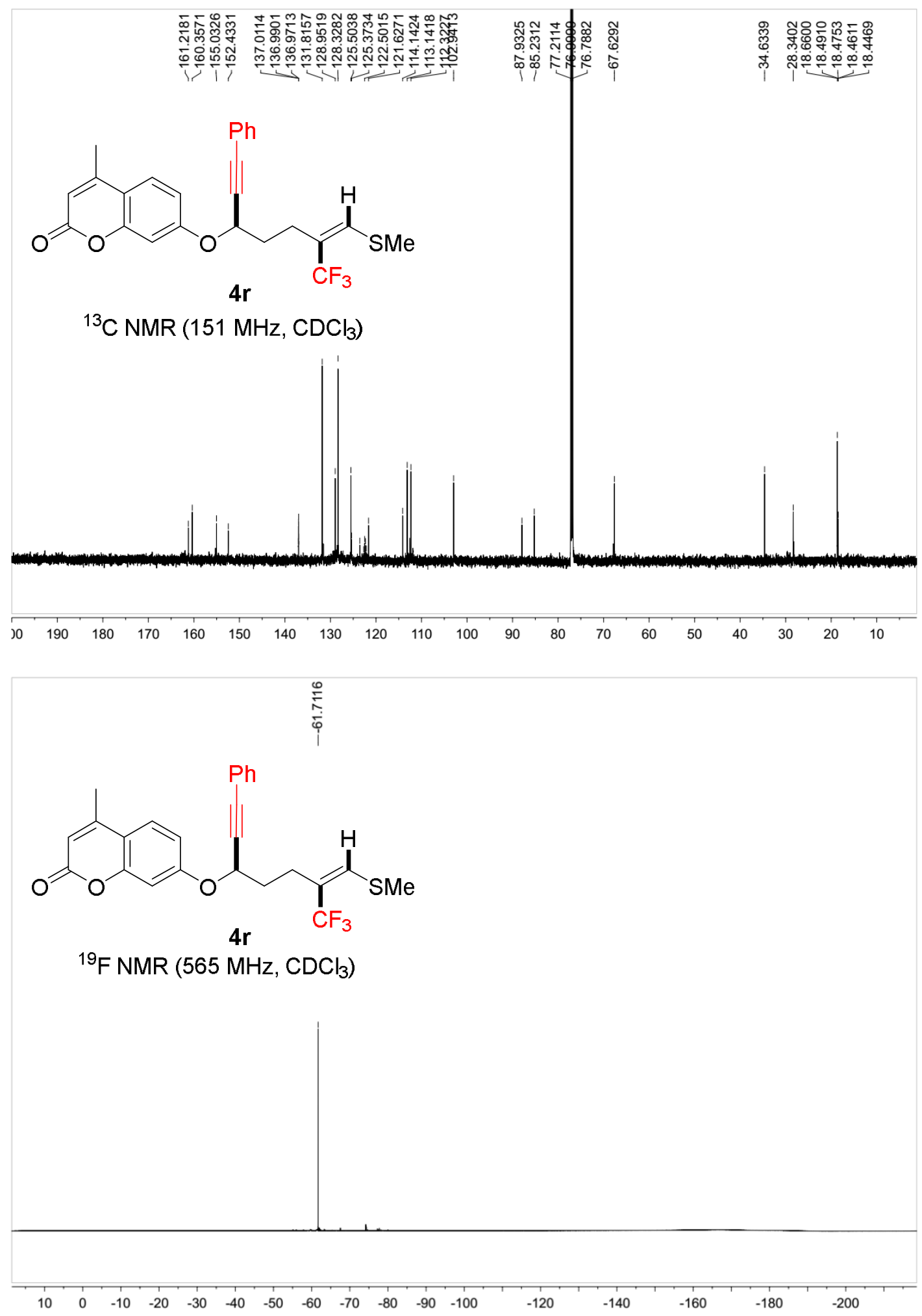


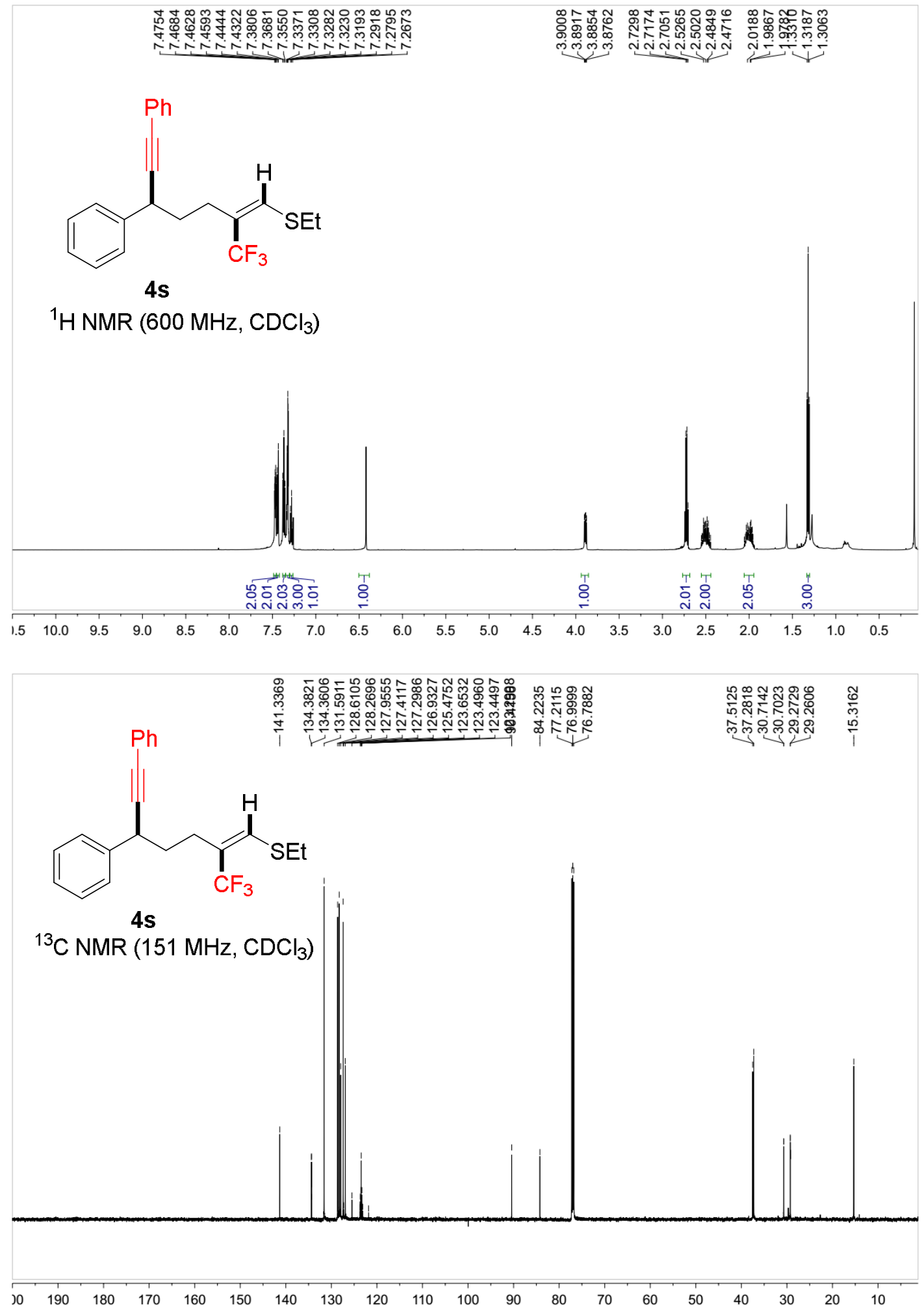




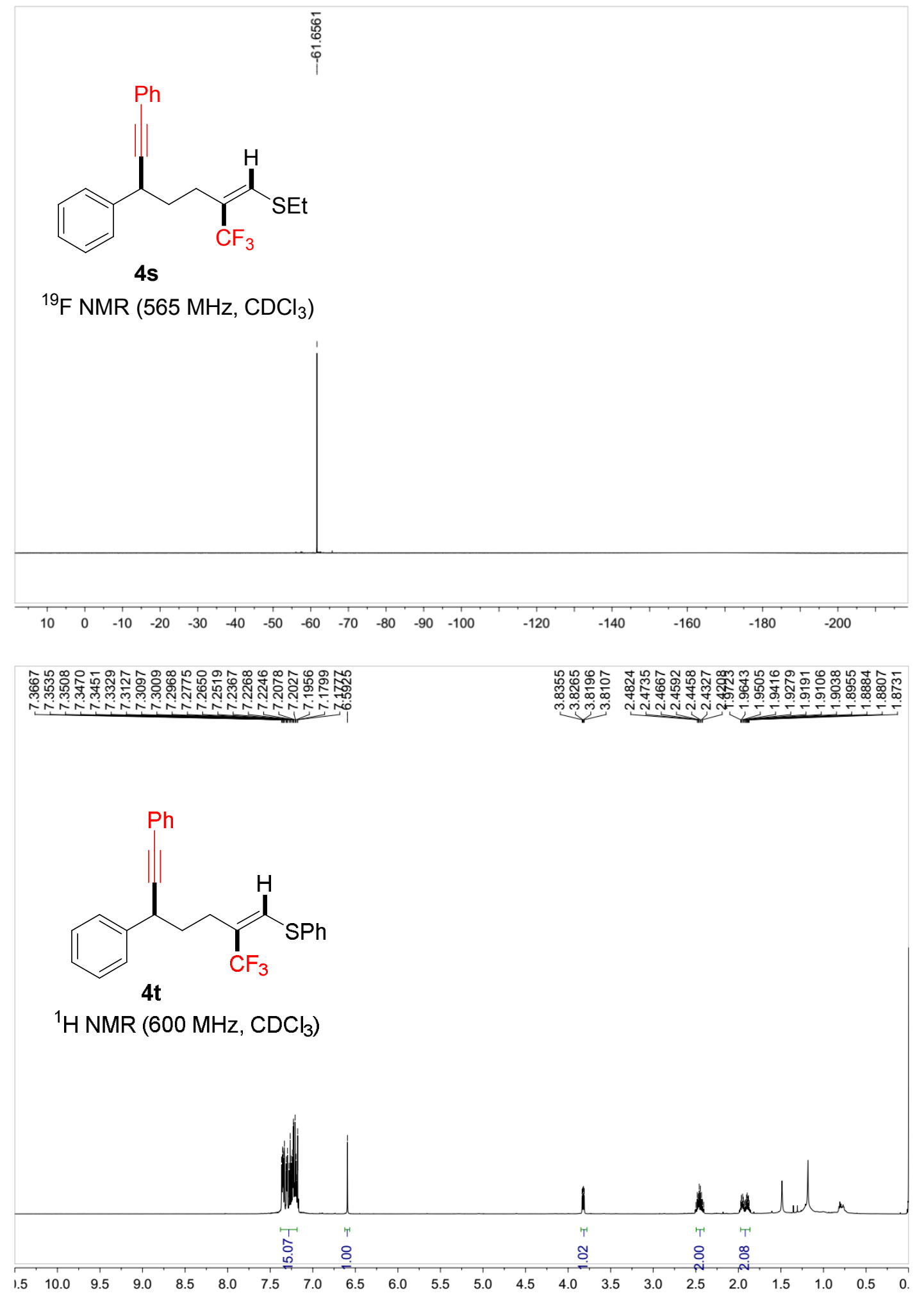




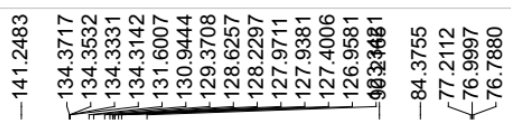

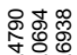

लिले

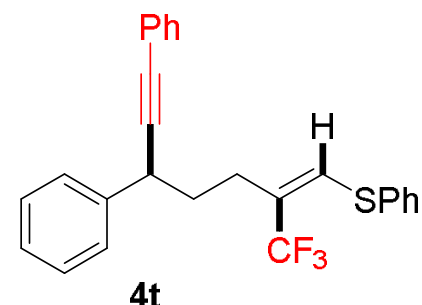

${ }^{13} \mathrm{C}$ NMR $\left(151 \mathrm{MHz}, \mathrm{CDCl}_{3}\right)$
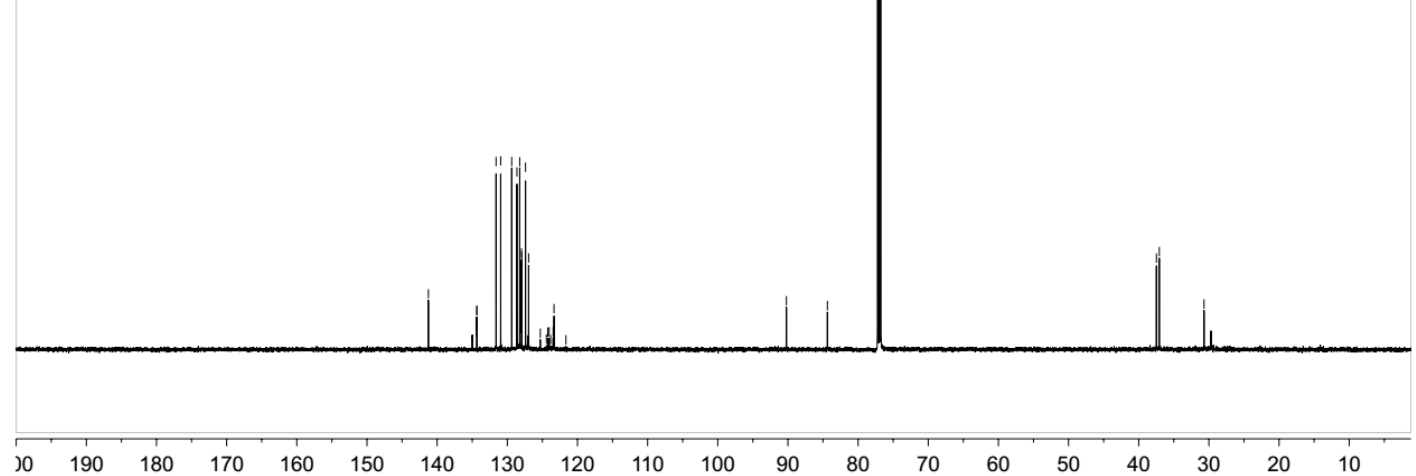

$$
\frac{\substack{5 \\ \frac{5}{\varphi}}}{1}
$$<smiles>FC(F)(F)C(=Cc1ccccc1)CCC(C#Cc1ccccc1)c1ccccc1</smiles>

${ }^{19} \mathrm{~F} \mathrm{NMR}\left(565 \mathrm{MHz}, \mathrm{CDCl}_{3}\right)$

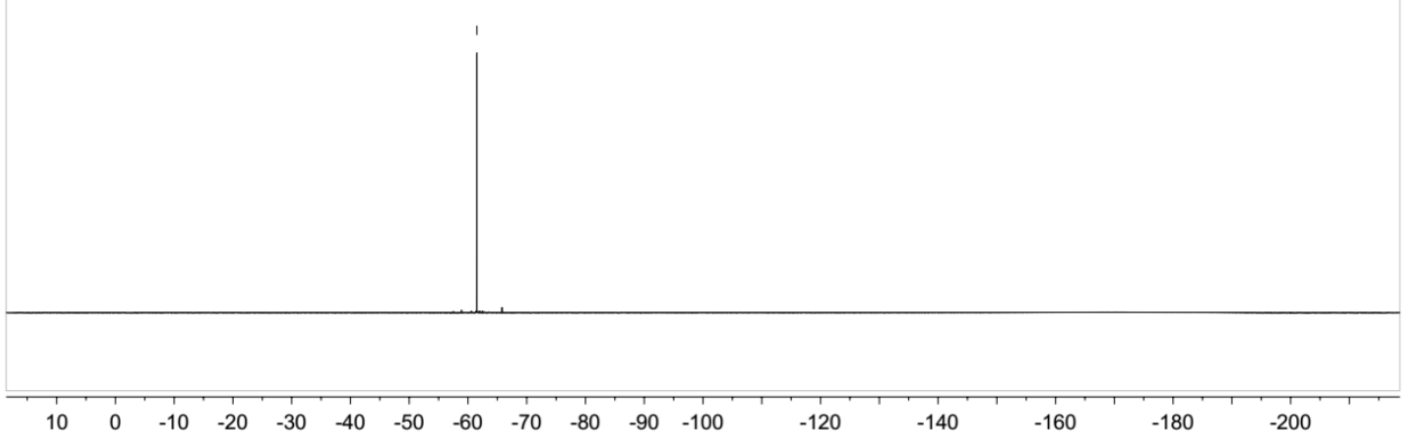




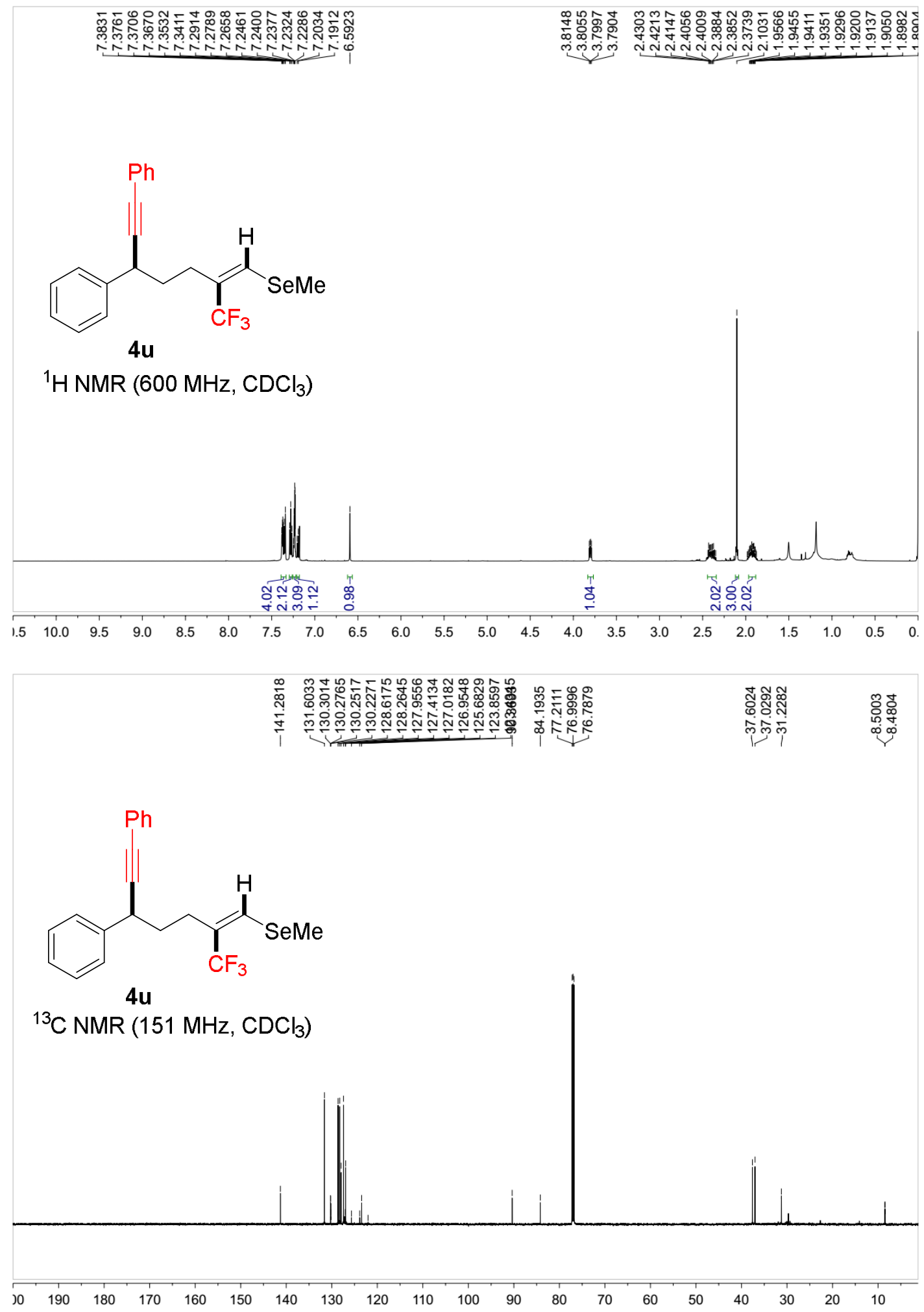



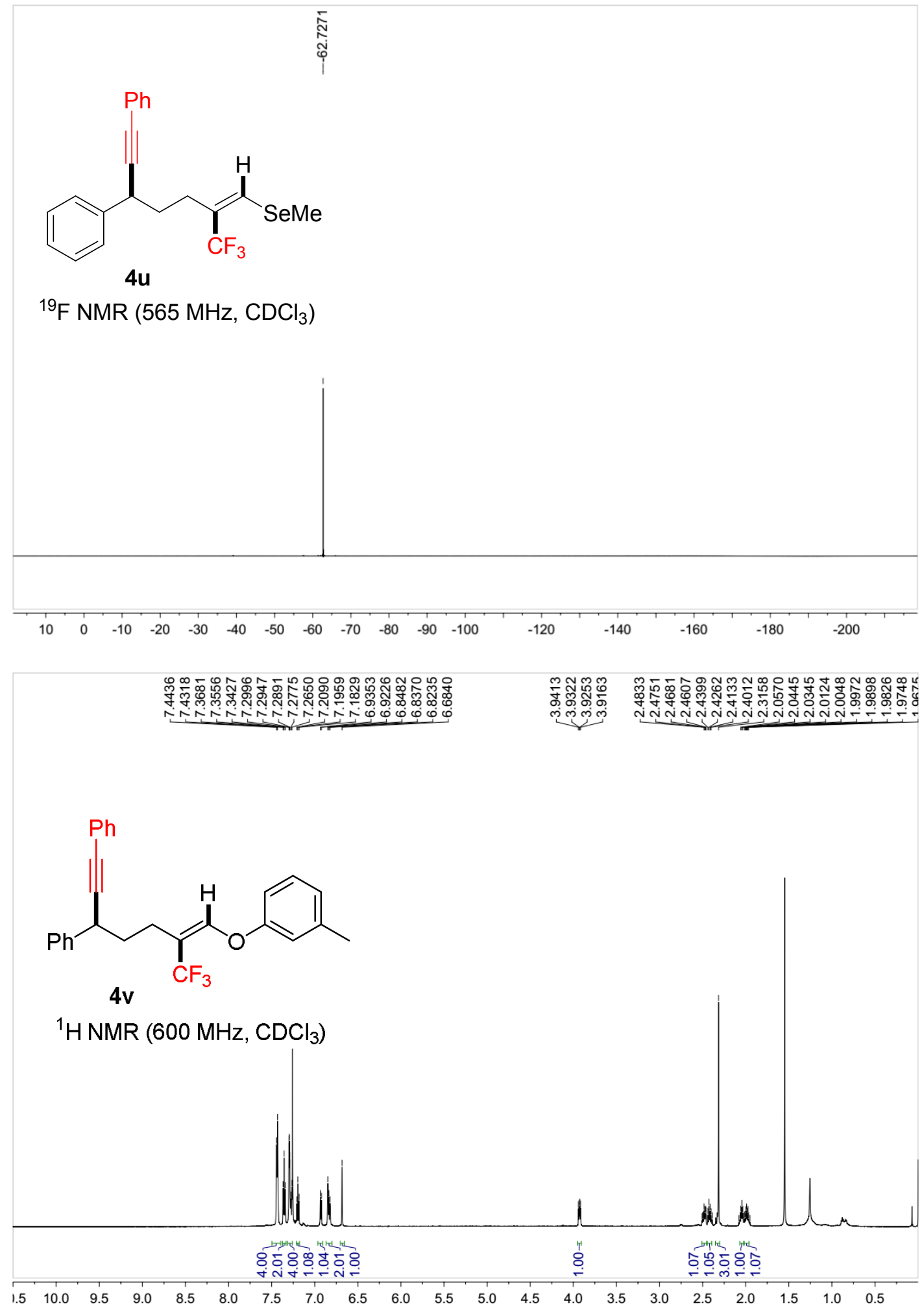

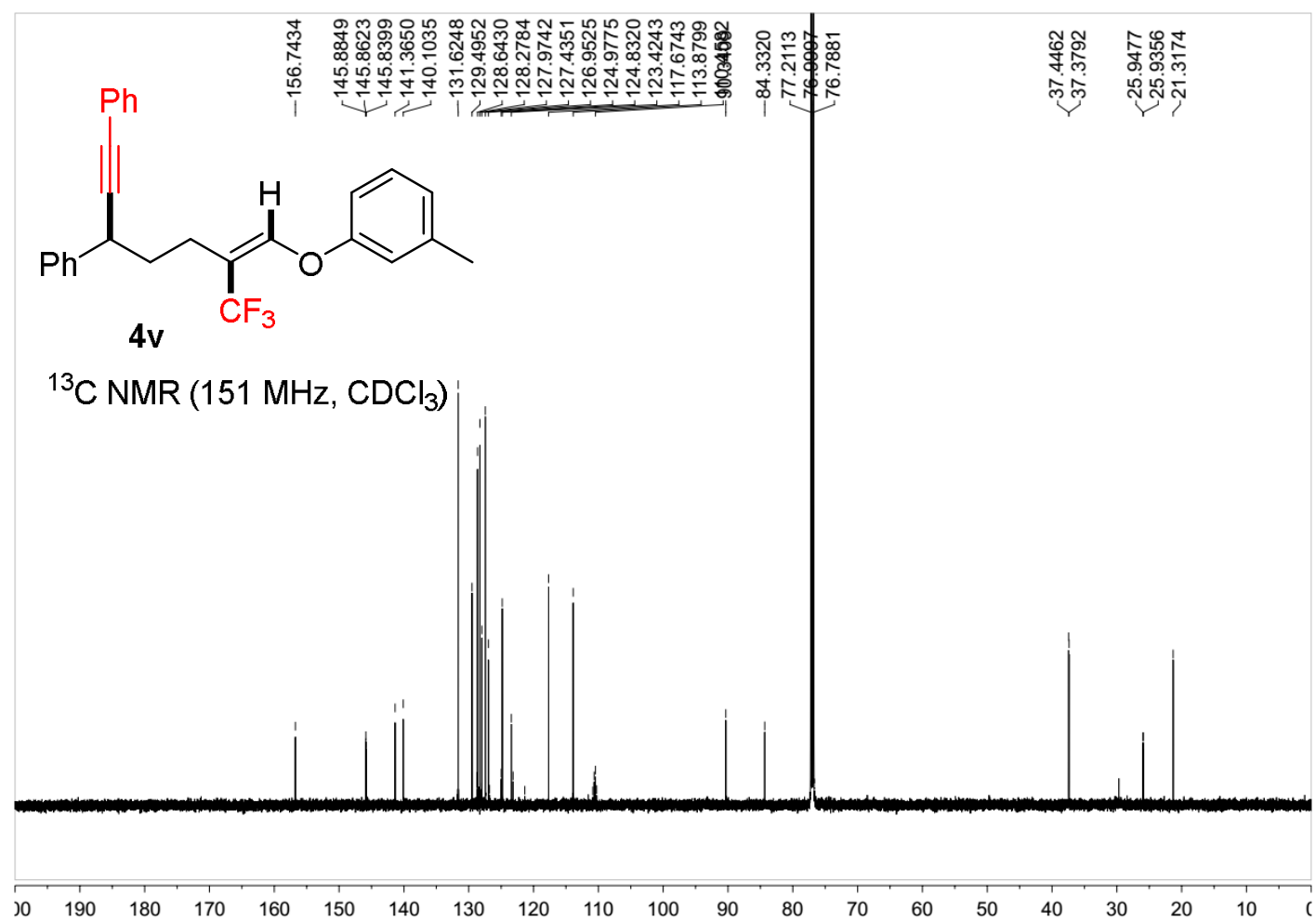

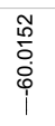<smiles>Cc1cccc(O/C=C(\CCC(C#Cc2ccccc2)c2ccccc2)C(F)(F)F)c1</smiles>

${ }^{19} \mathrm{~F} \mathrm{NMR}\left(565 \mathrm{MHz}, \mathrm{CDCl}_{3}\right)$

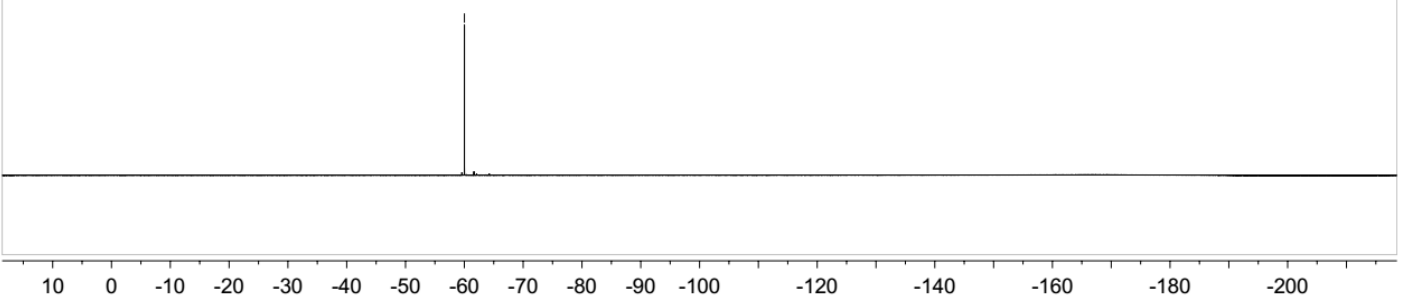




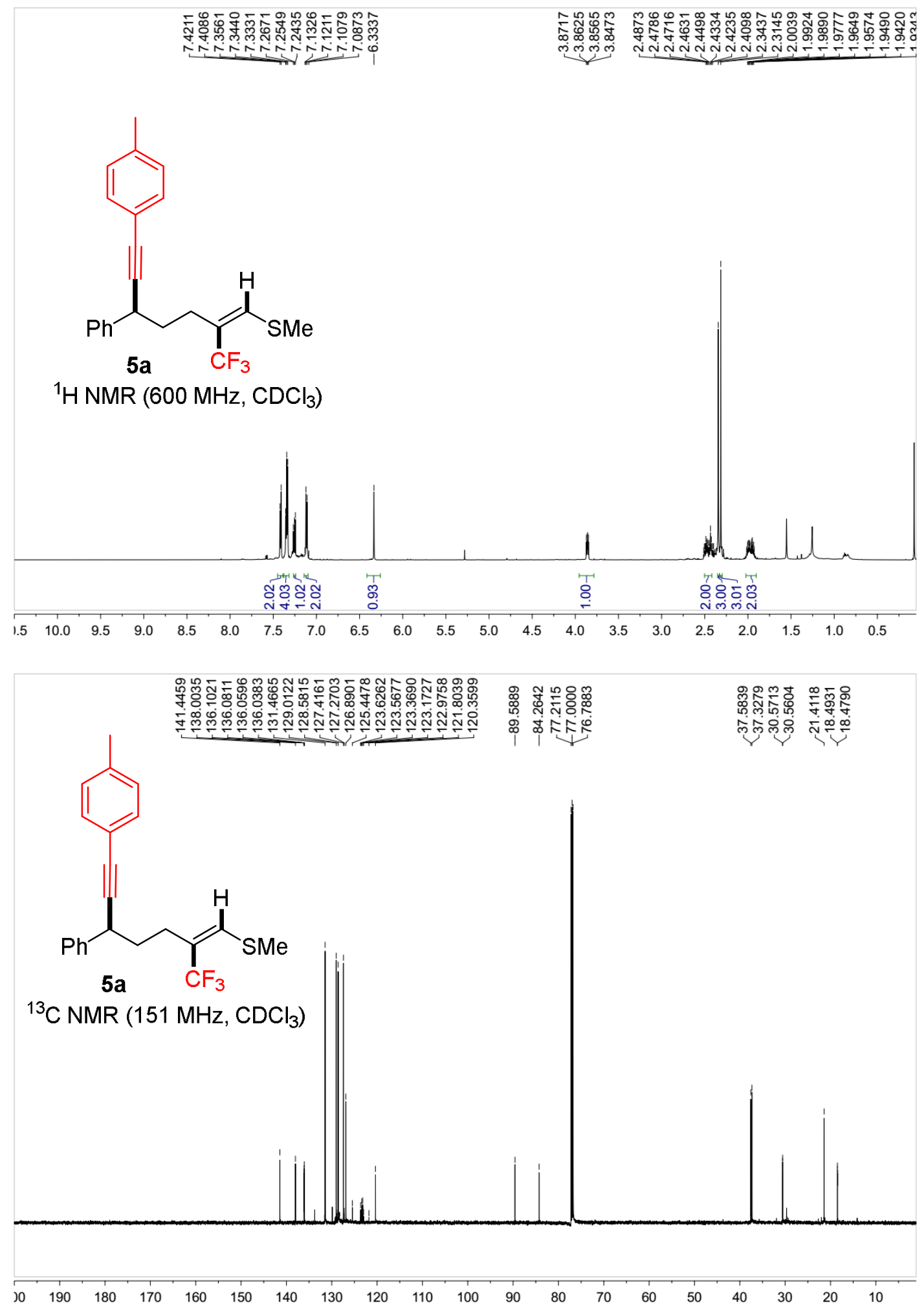



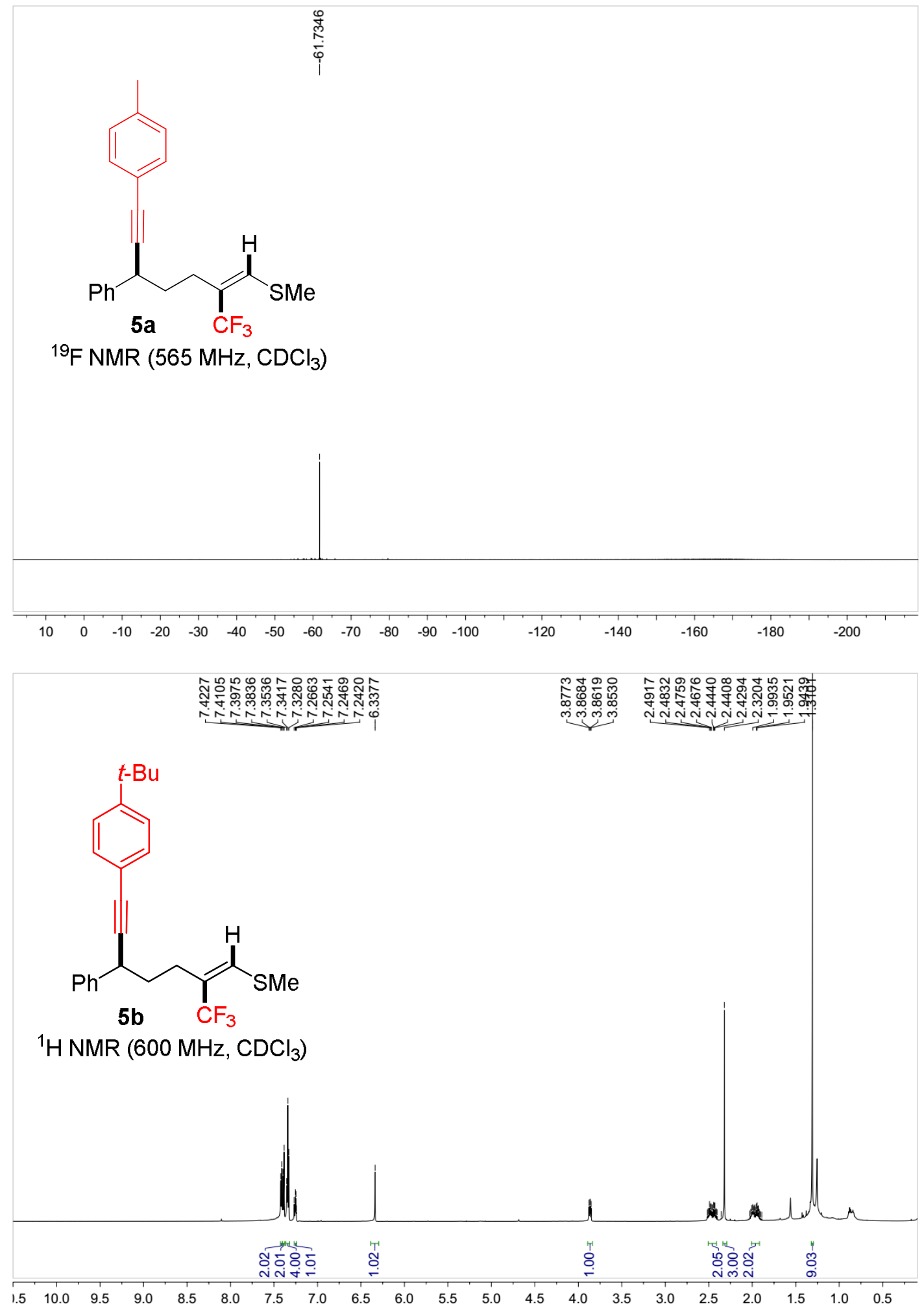


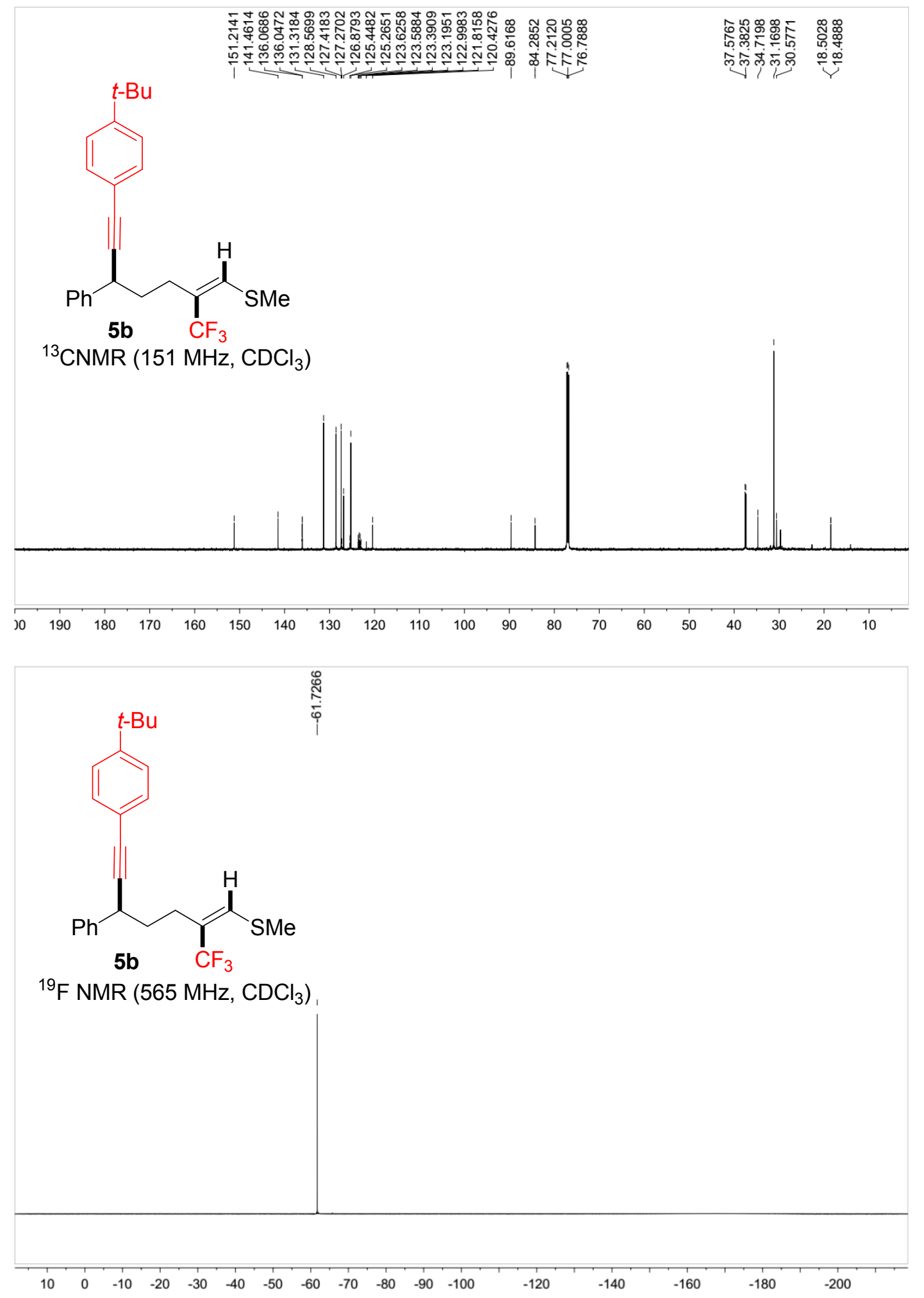




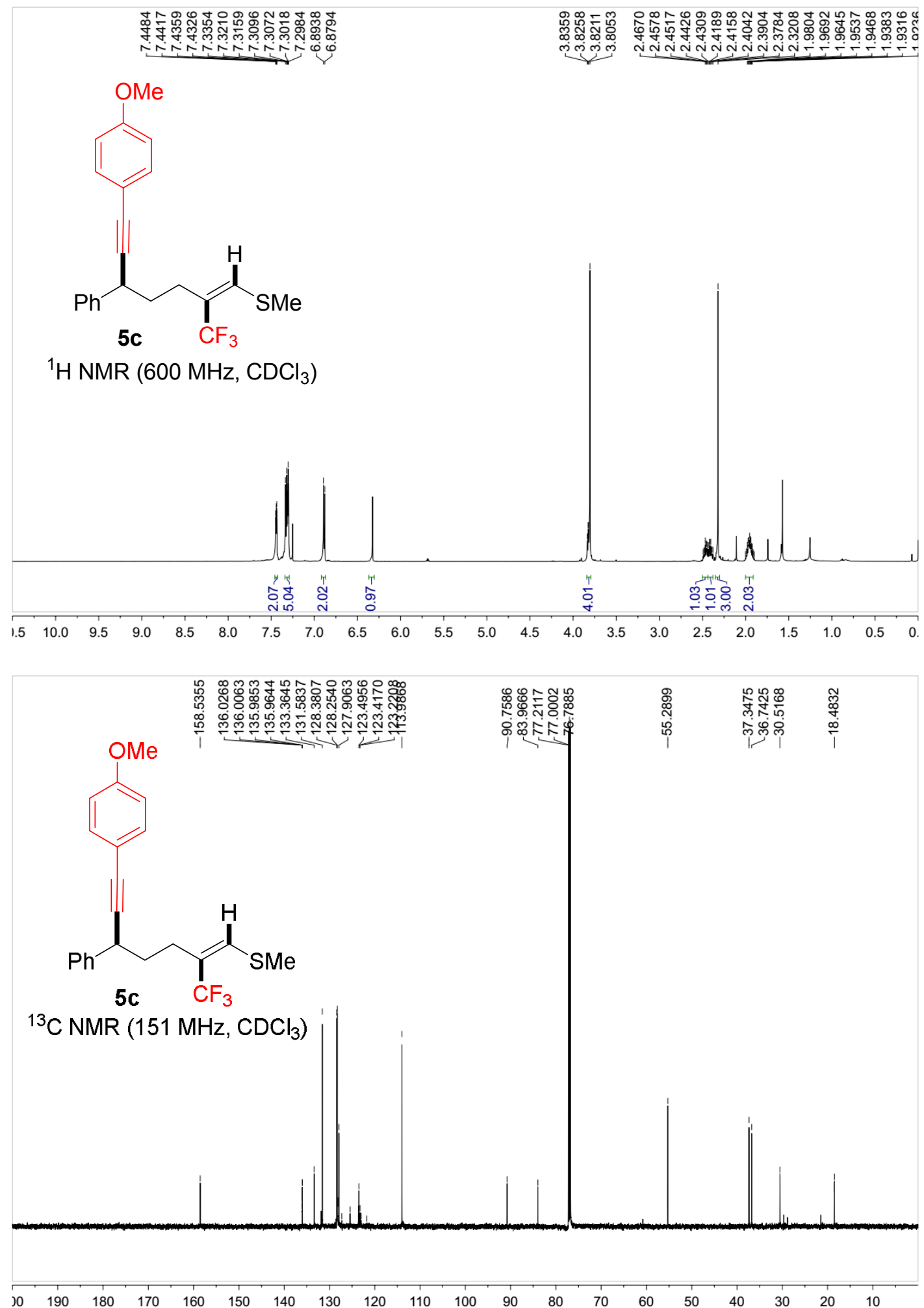




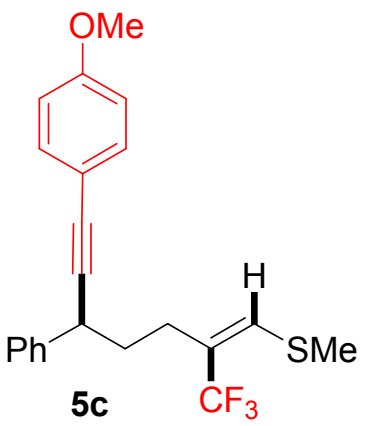

$\frac{n}{0}$

$\left.{ }^{19} \mathrm{~F} \mathrm{NMR} \mathrm{(565} \mathrm{MHz,} \mathrm{CDCl}_{3}\right)$
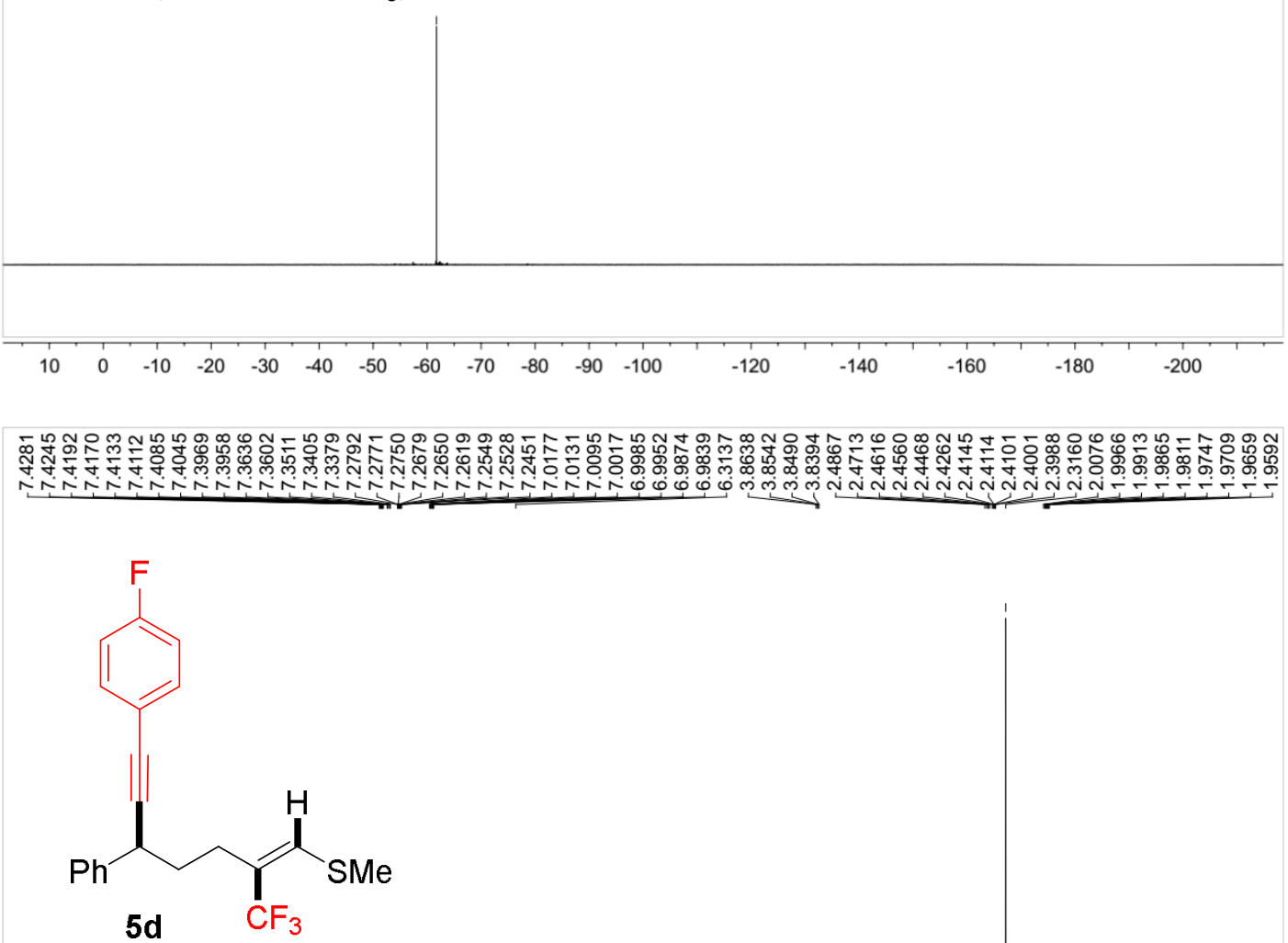

${ }^{1} \mathrm{H}$ NMR $\left(600 \mathrm{MHz}, \mathrm{CDCl}_{3}\right)$

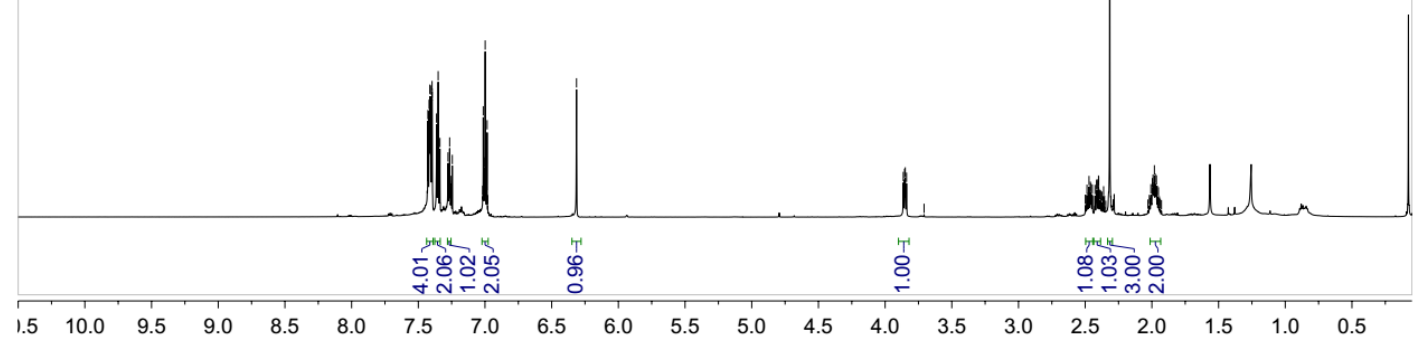



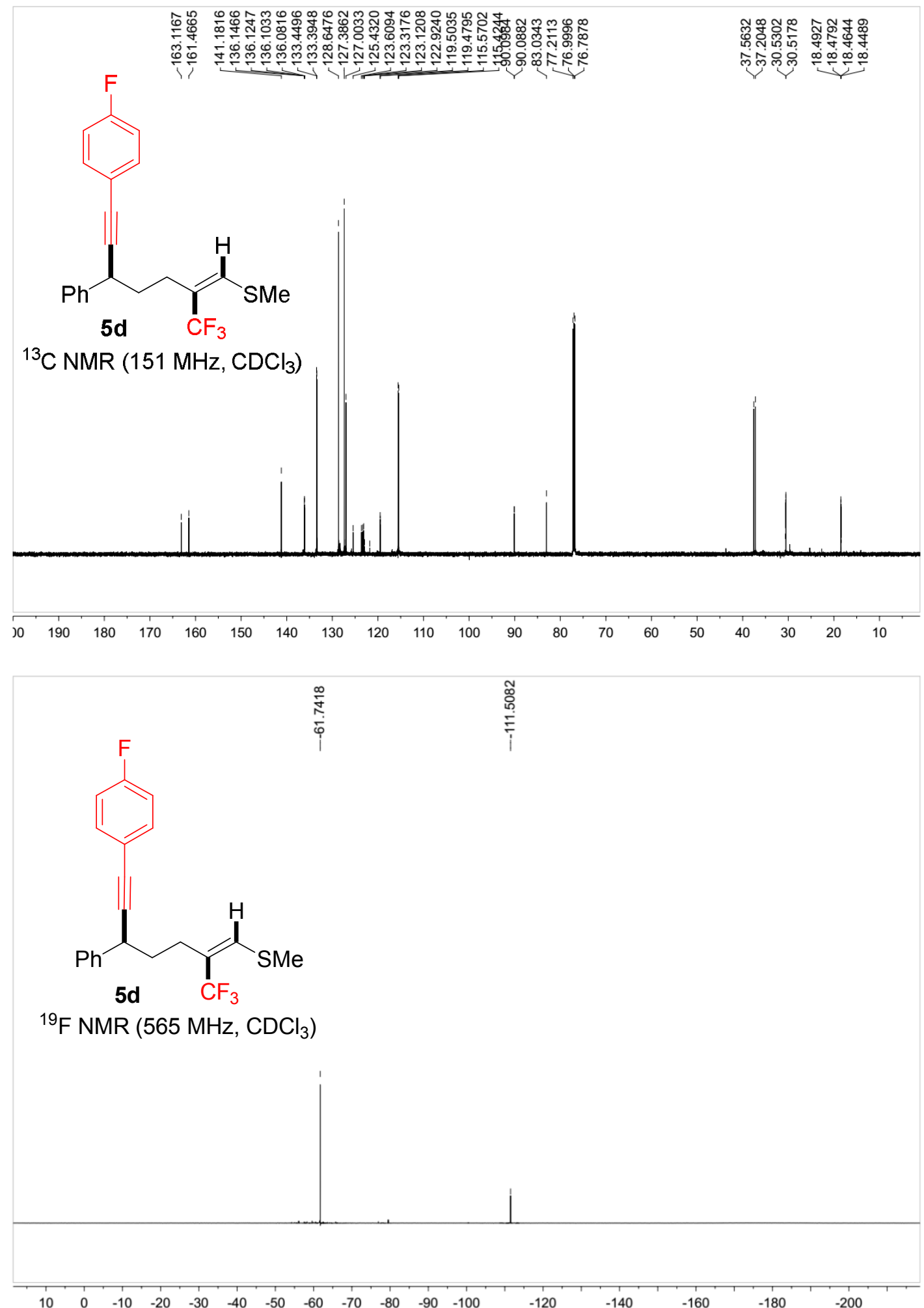

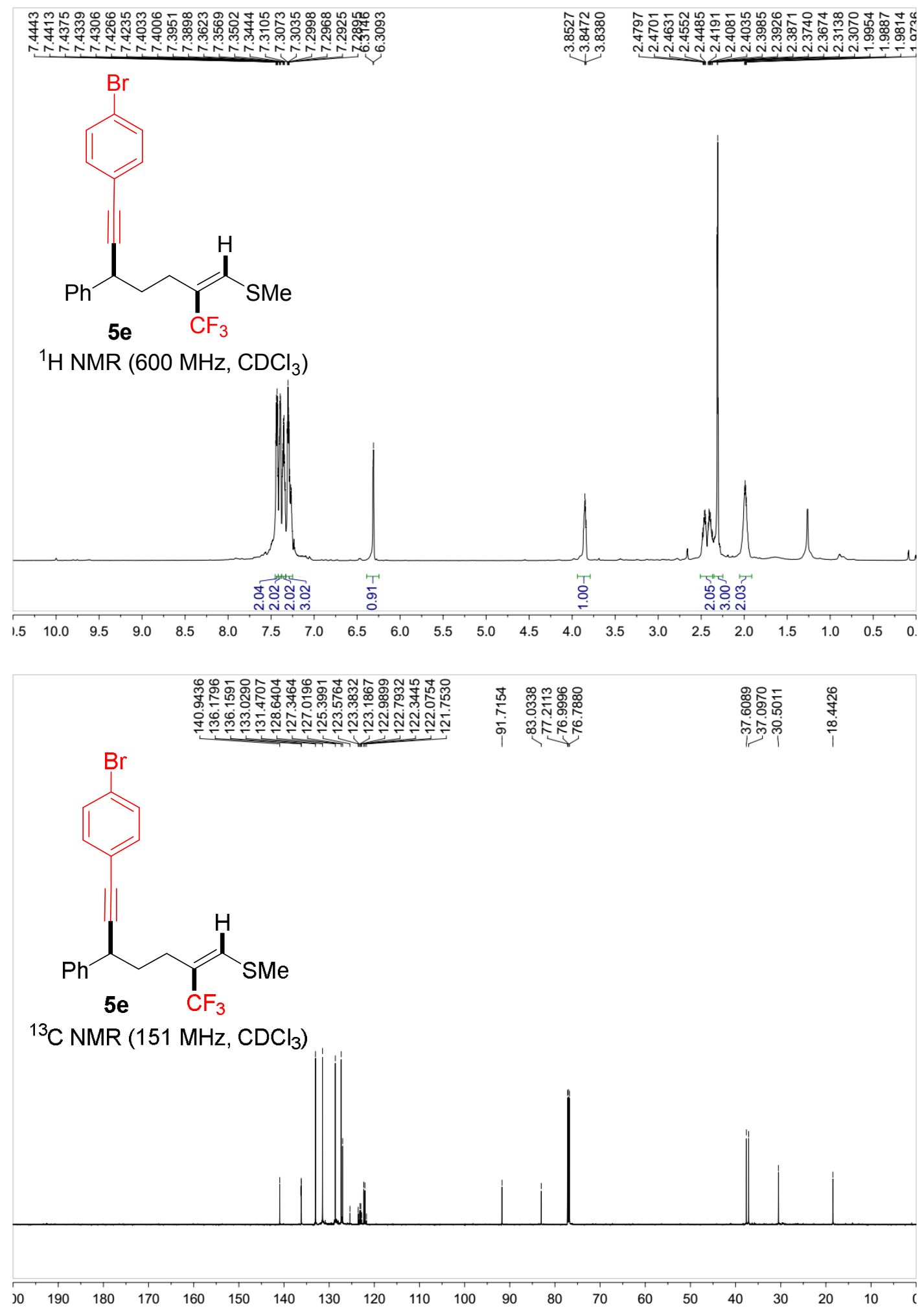

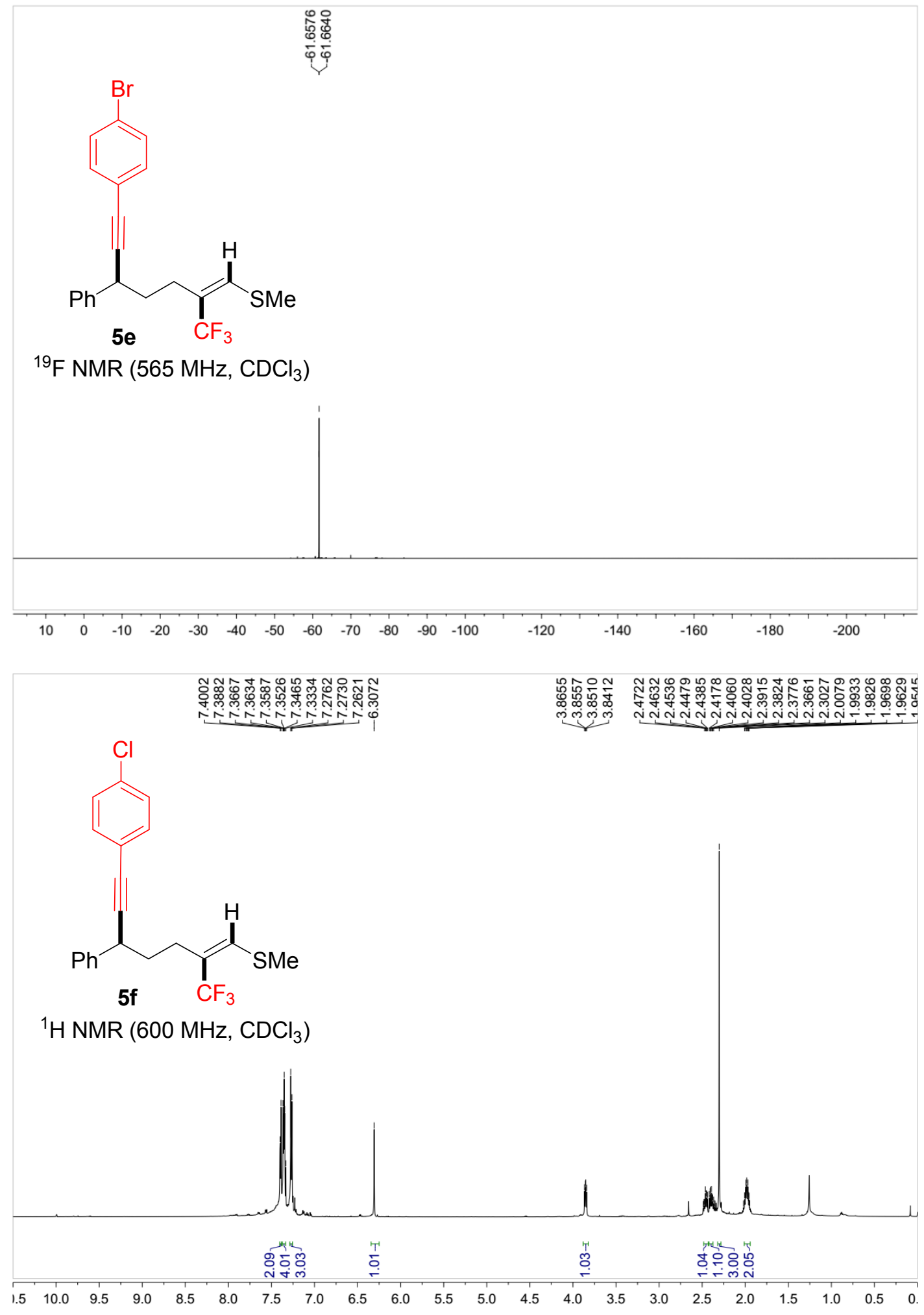


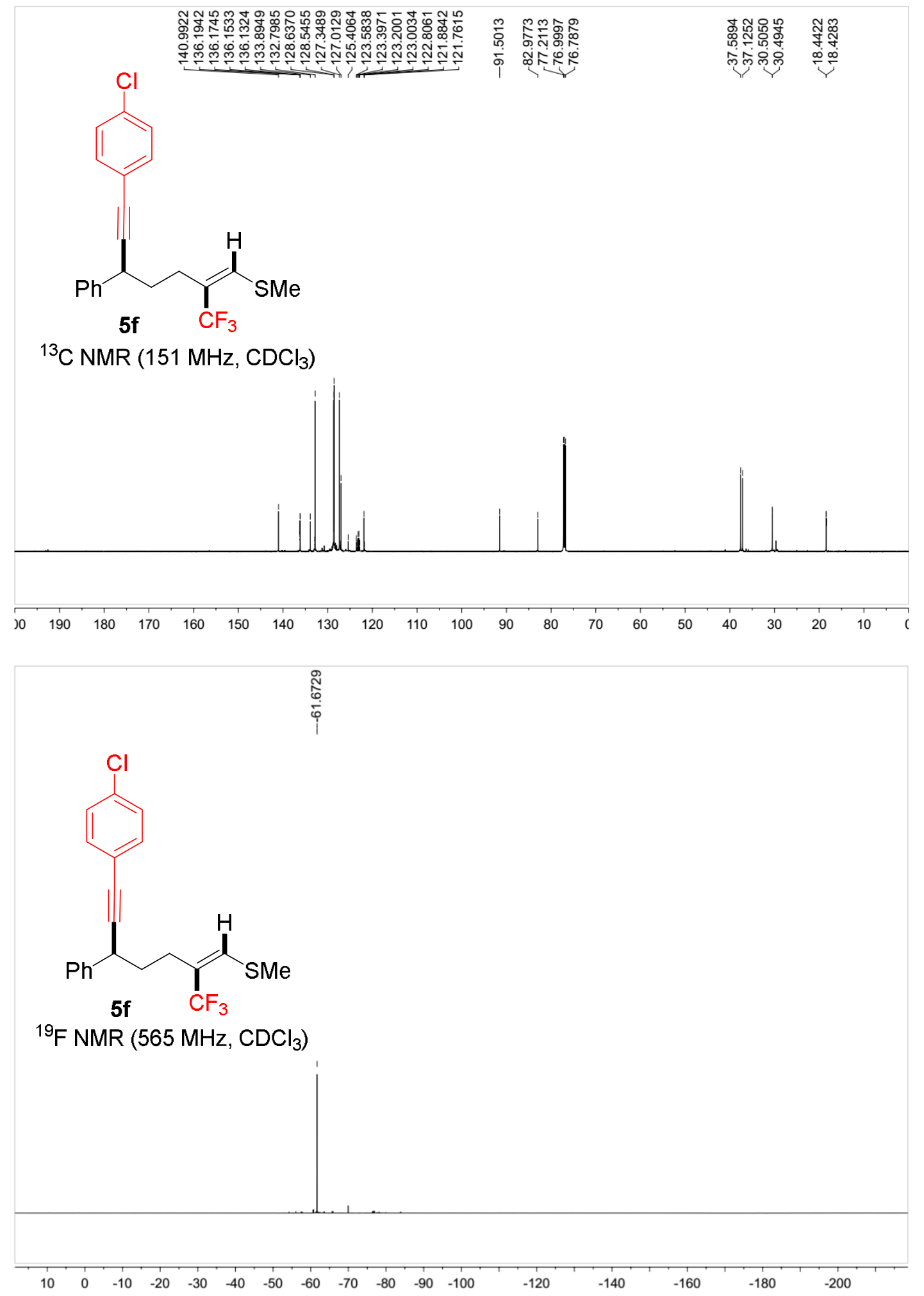



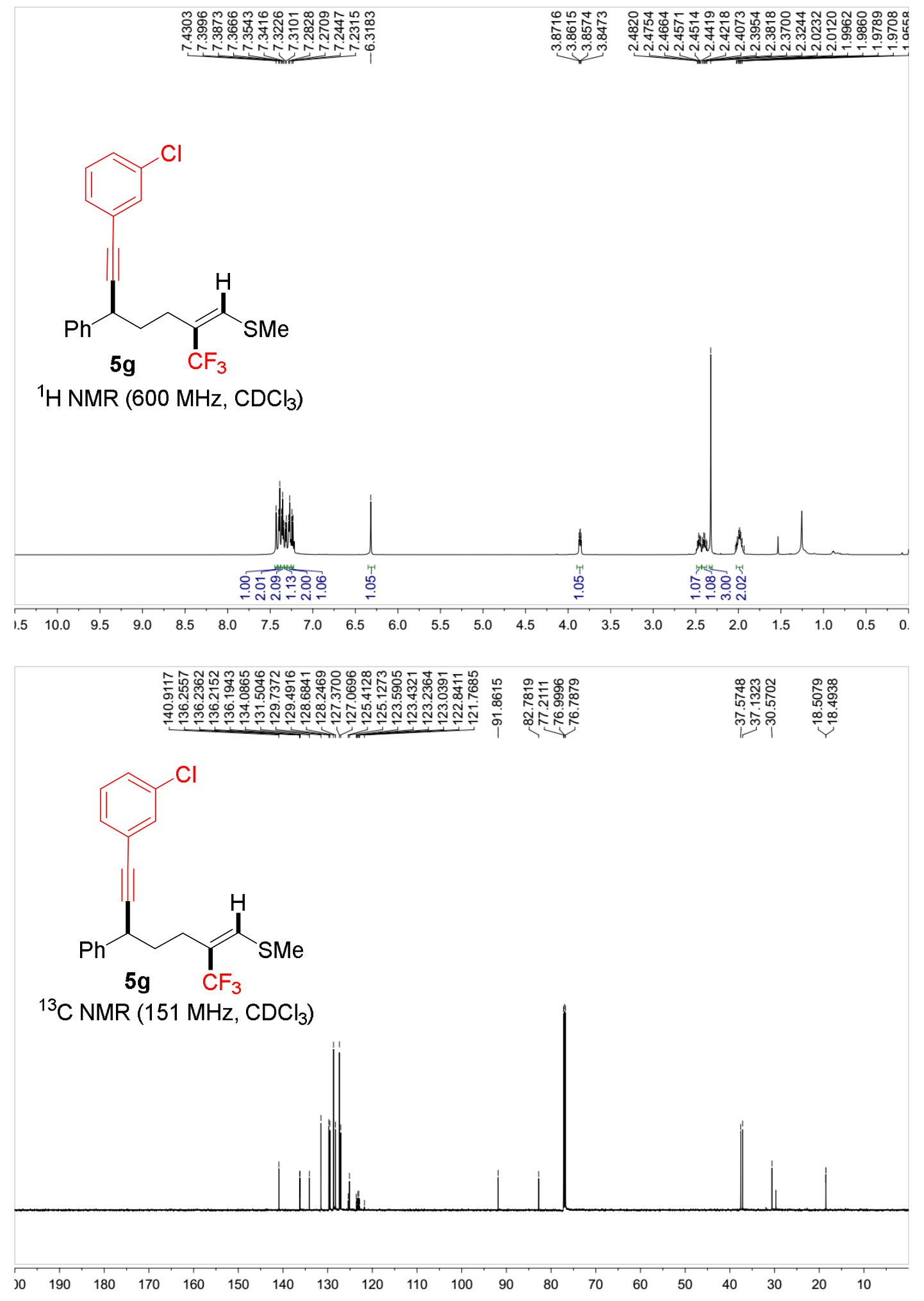

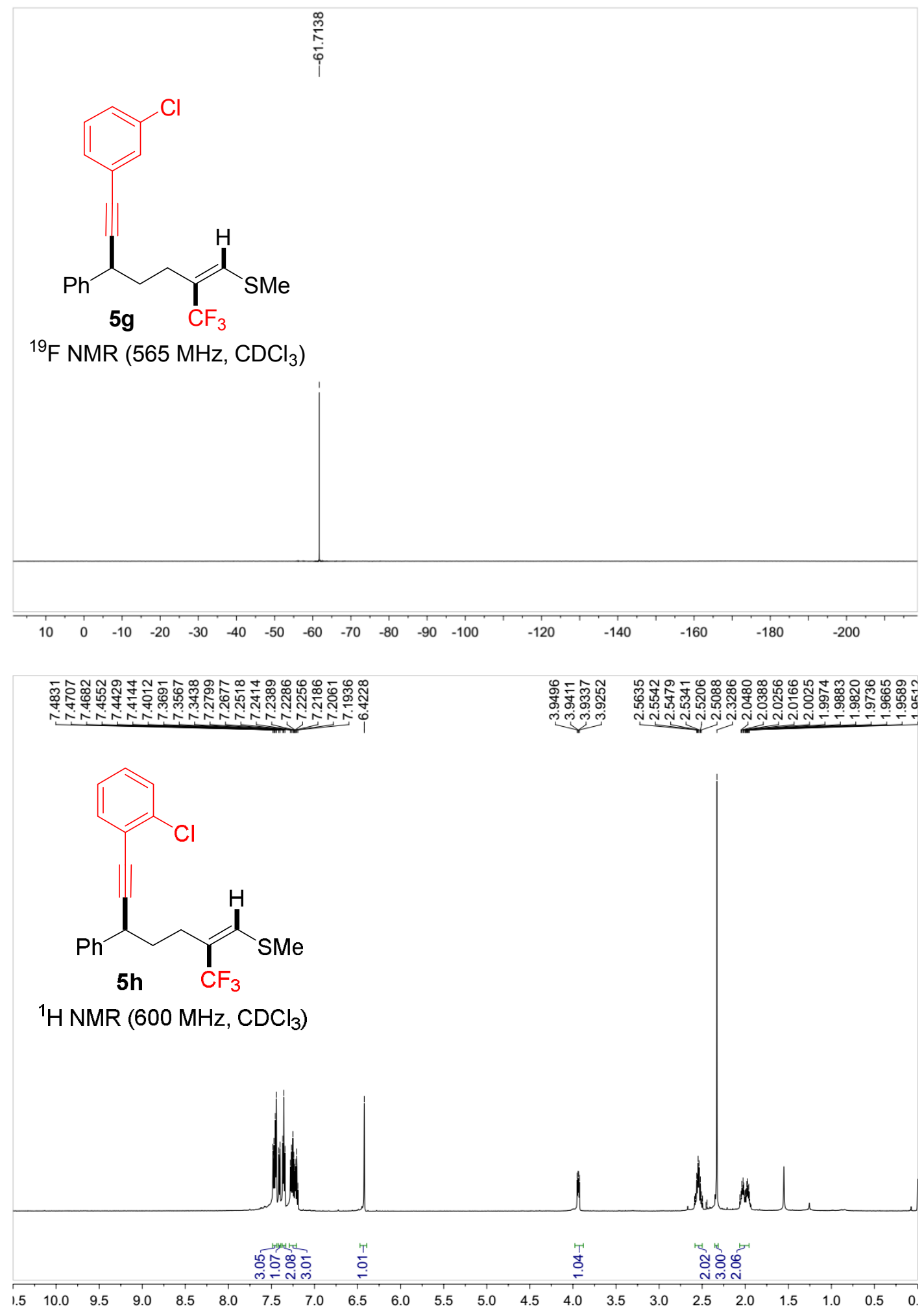


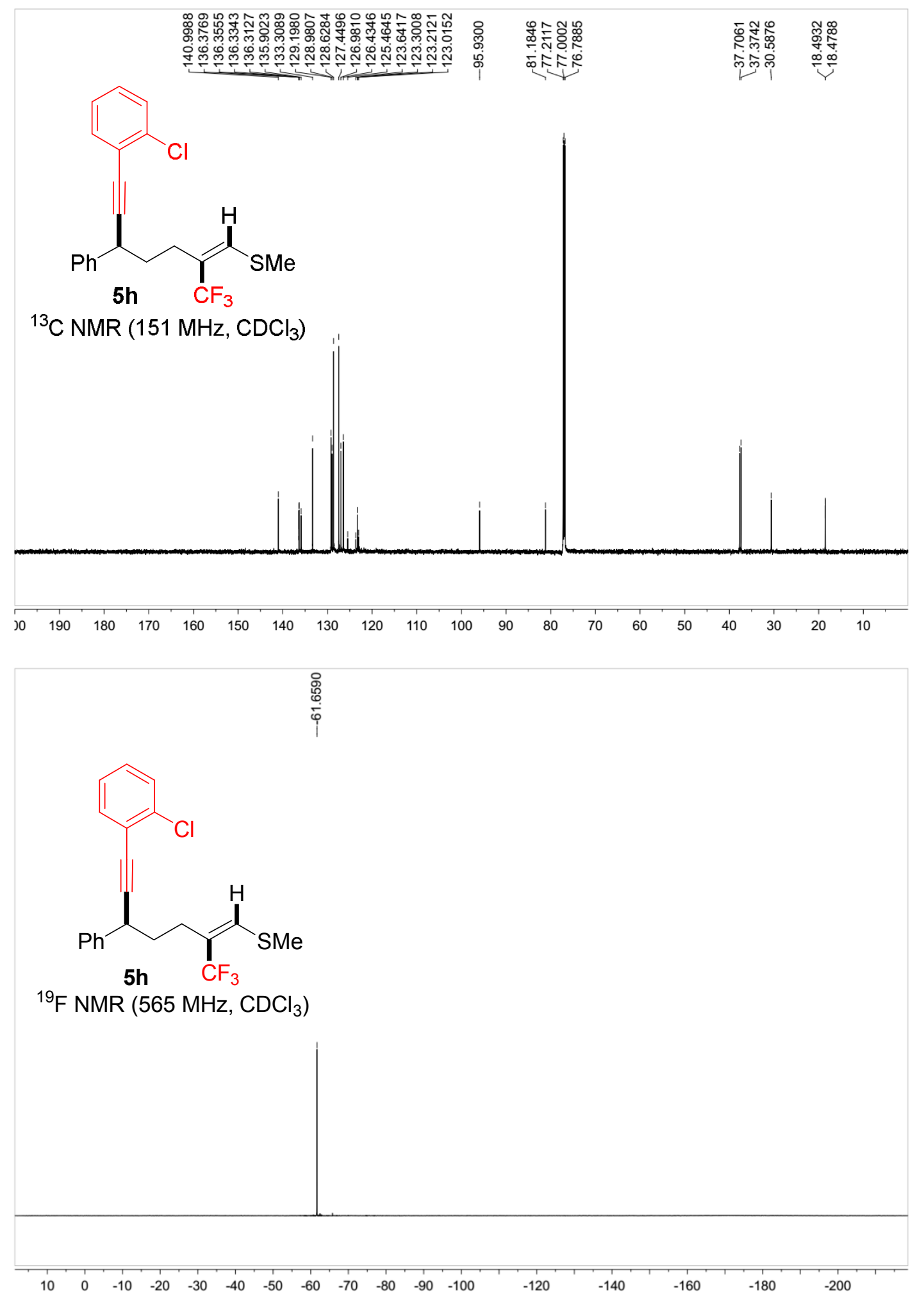




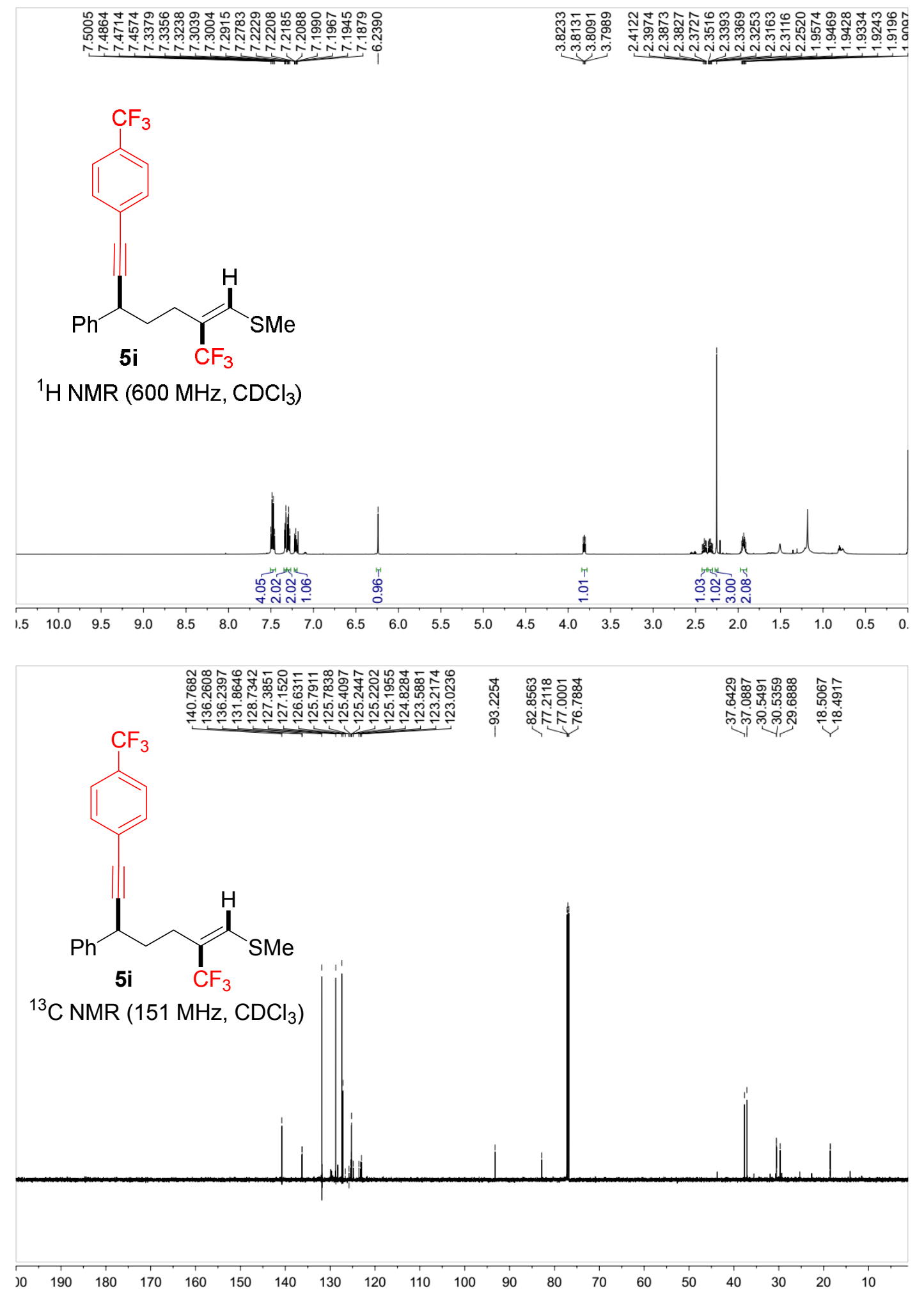




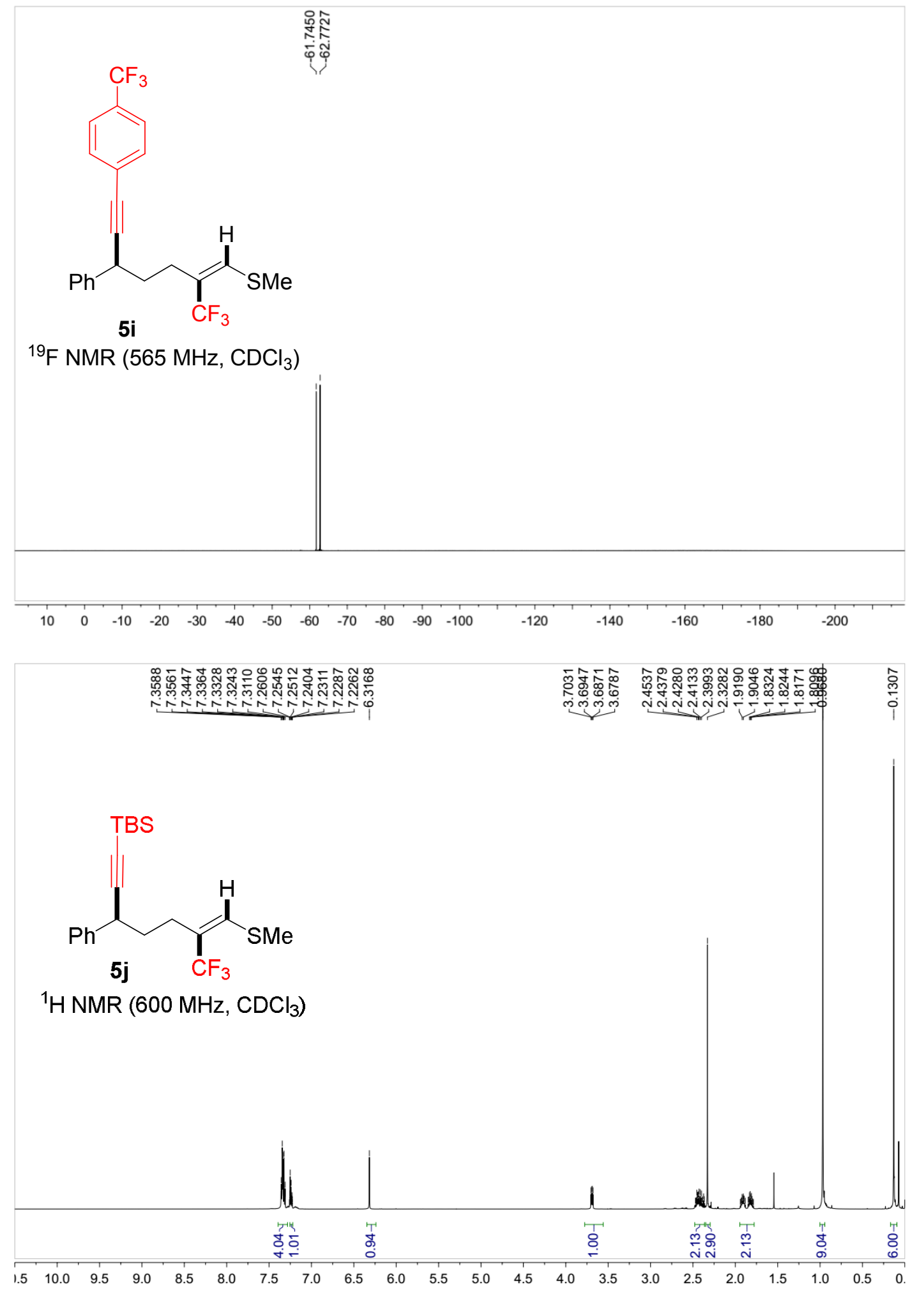




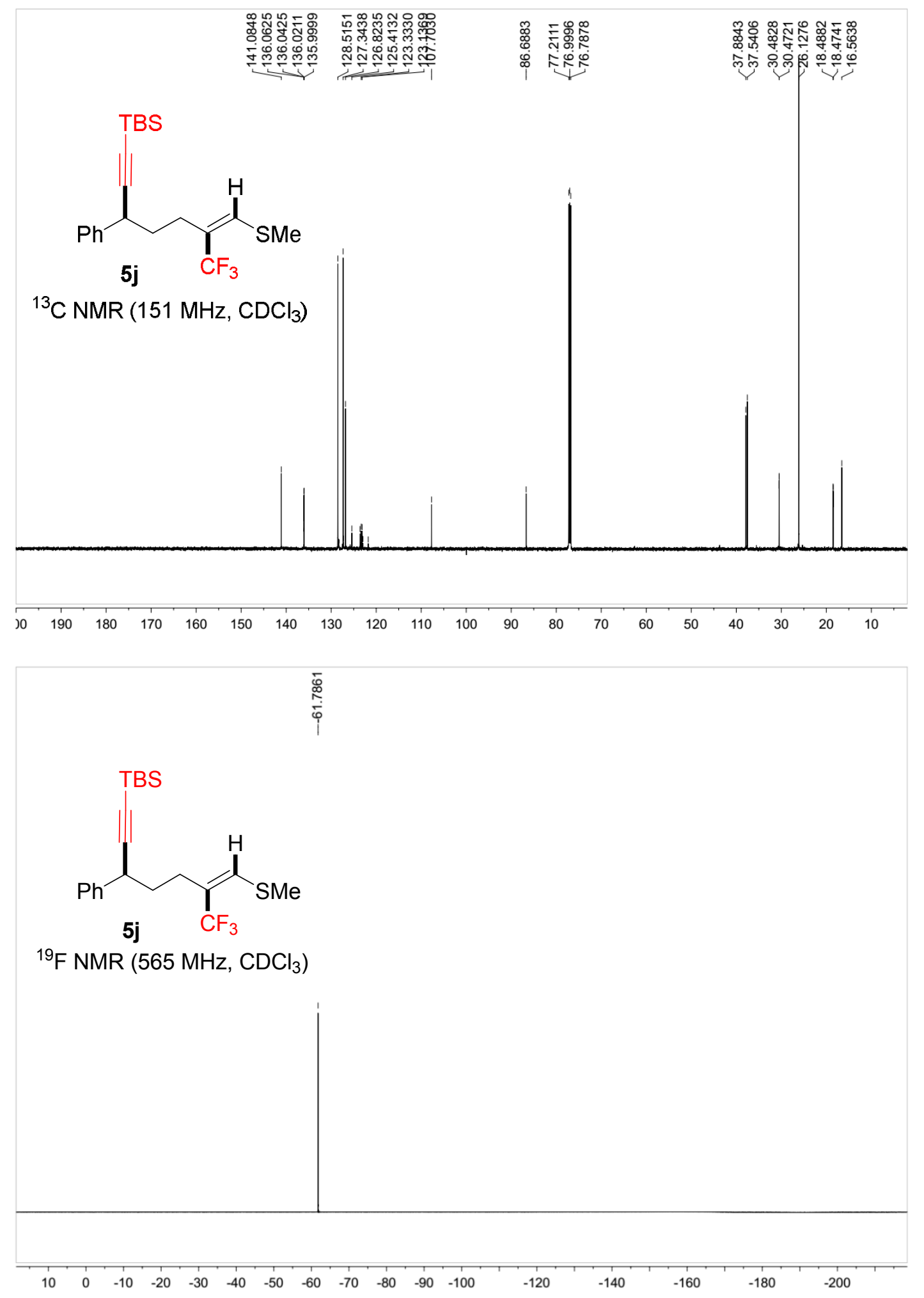



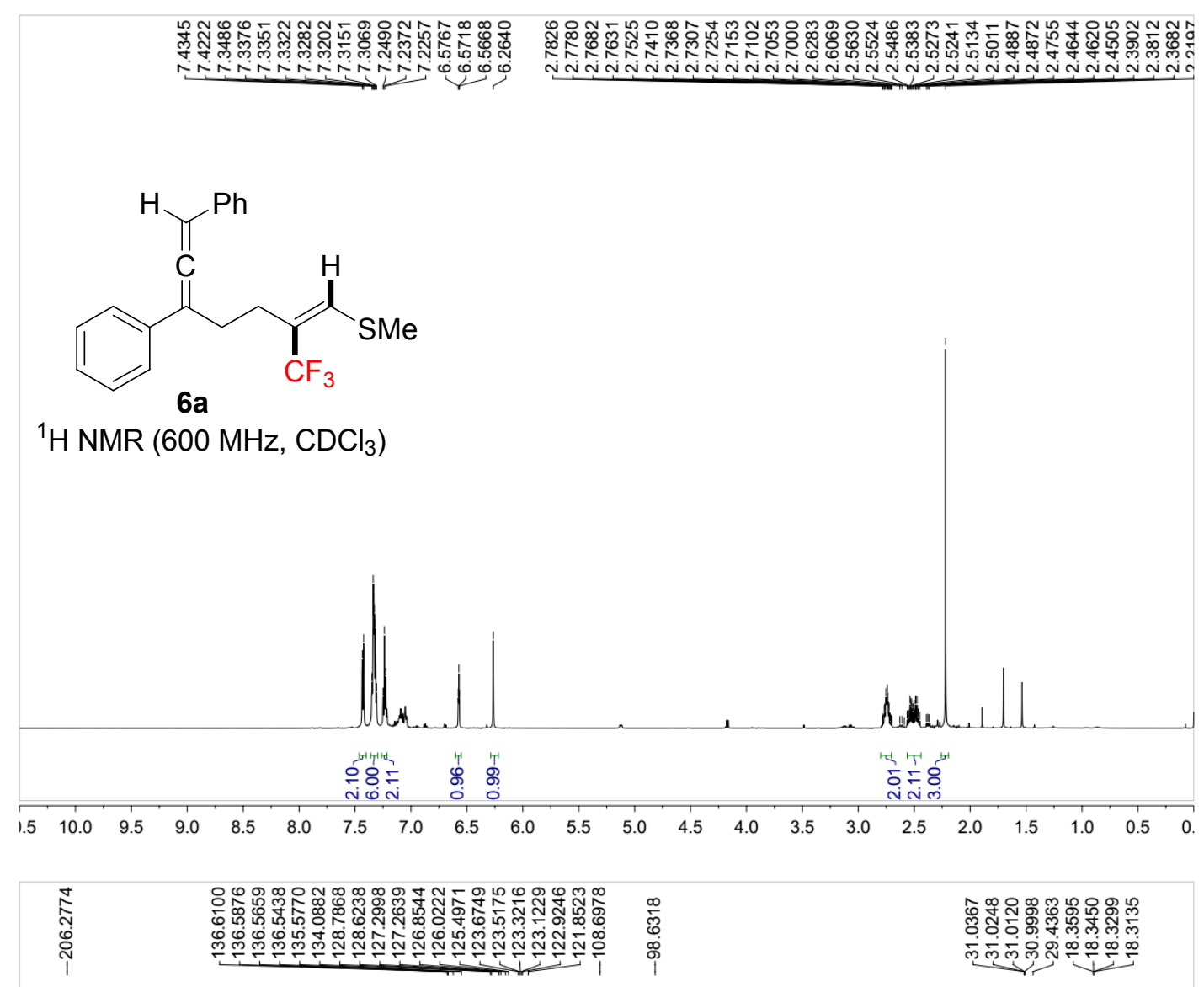<smiles>C/C(=C/CC(=C=Cc1ccccc1)c1ccccc1)C(F)(F)F</smiles>

${ }^{13} \mathrm{C}$ NMR $\left(151 \mathrm{MHz}, \mathrm{CDCl}_{3}\right)$

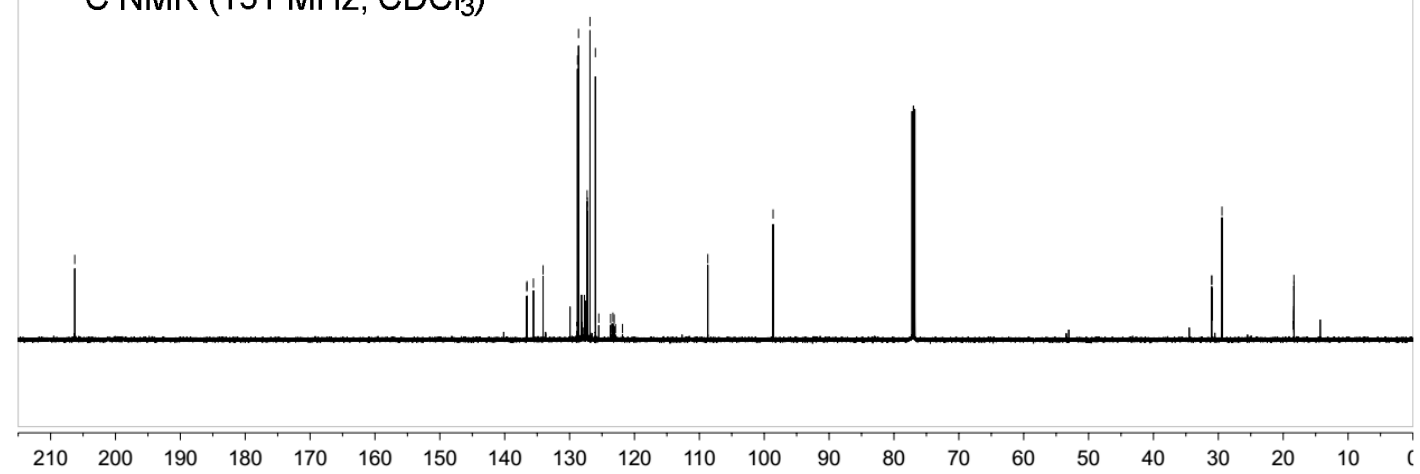




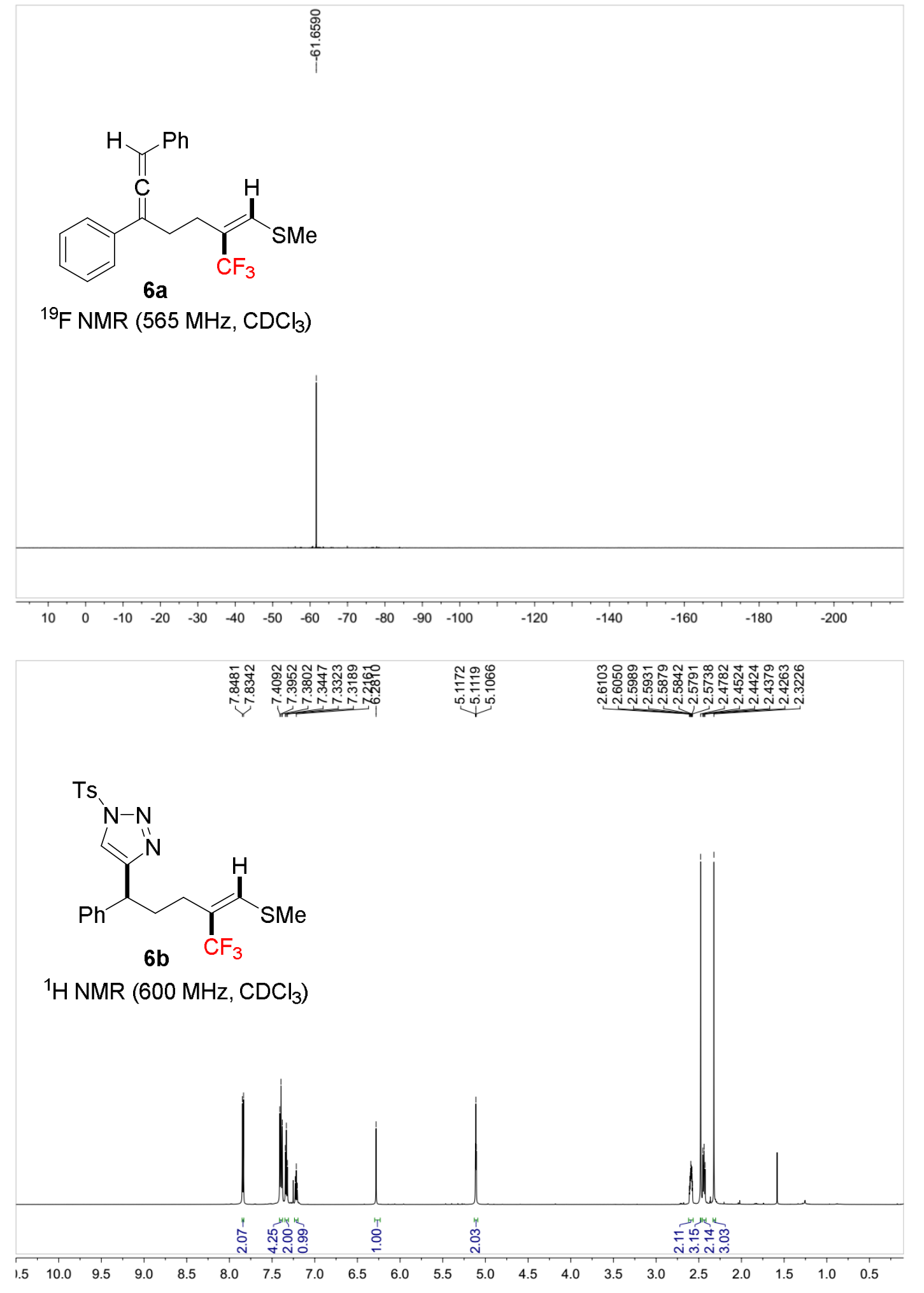




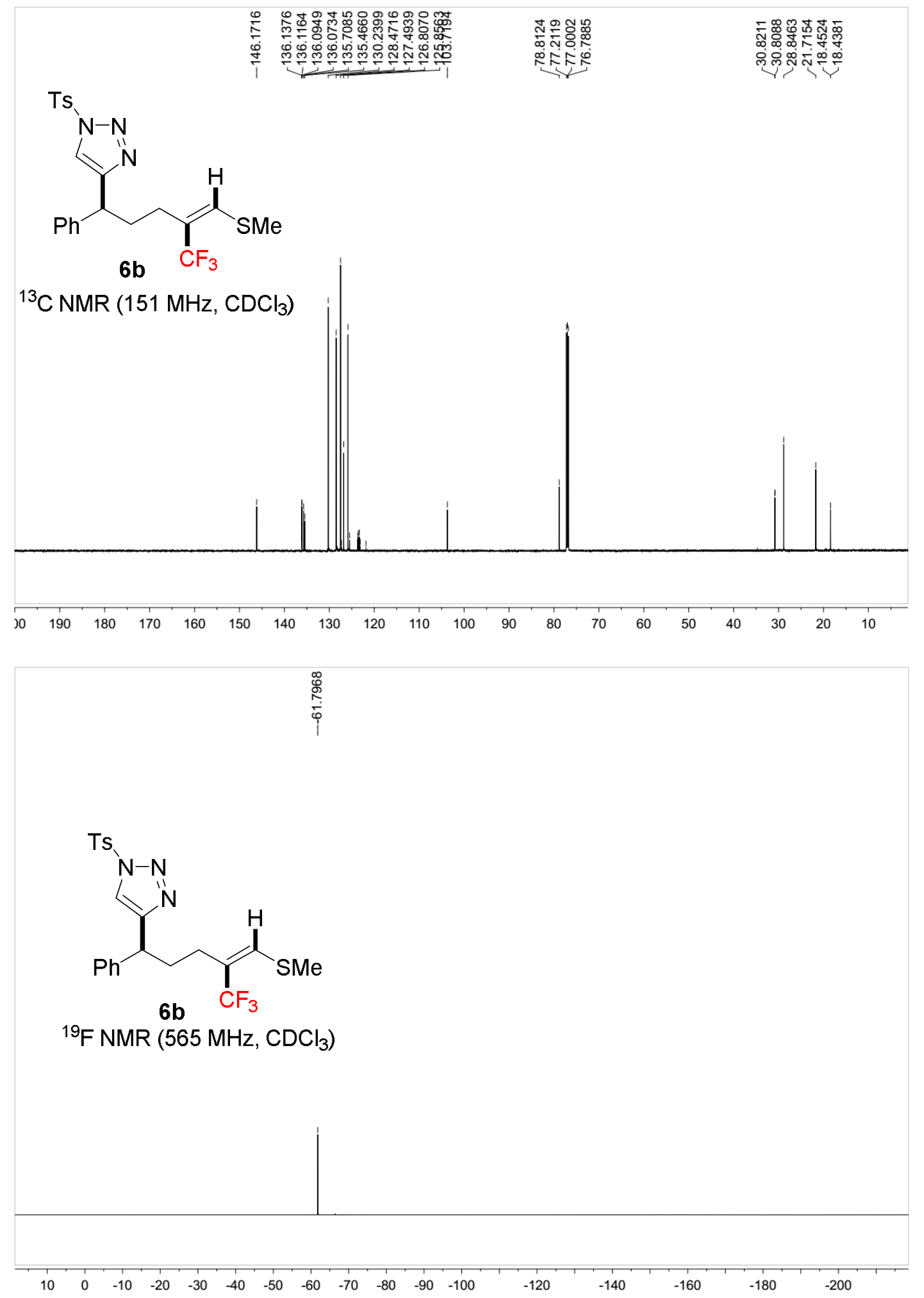




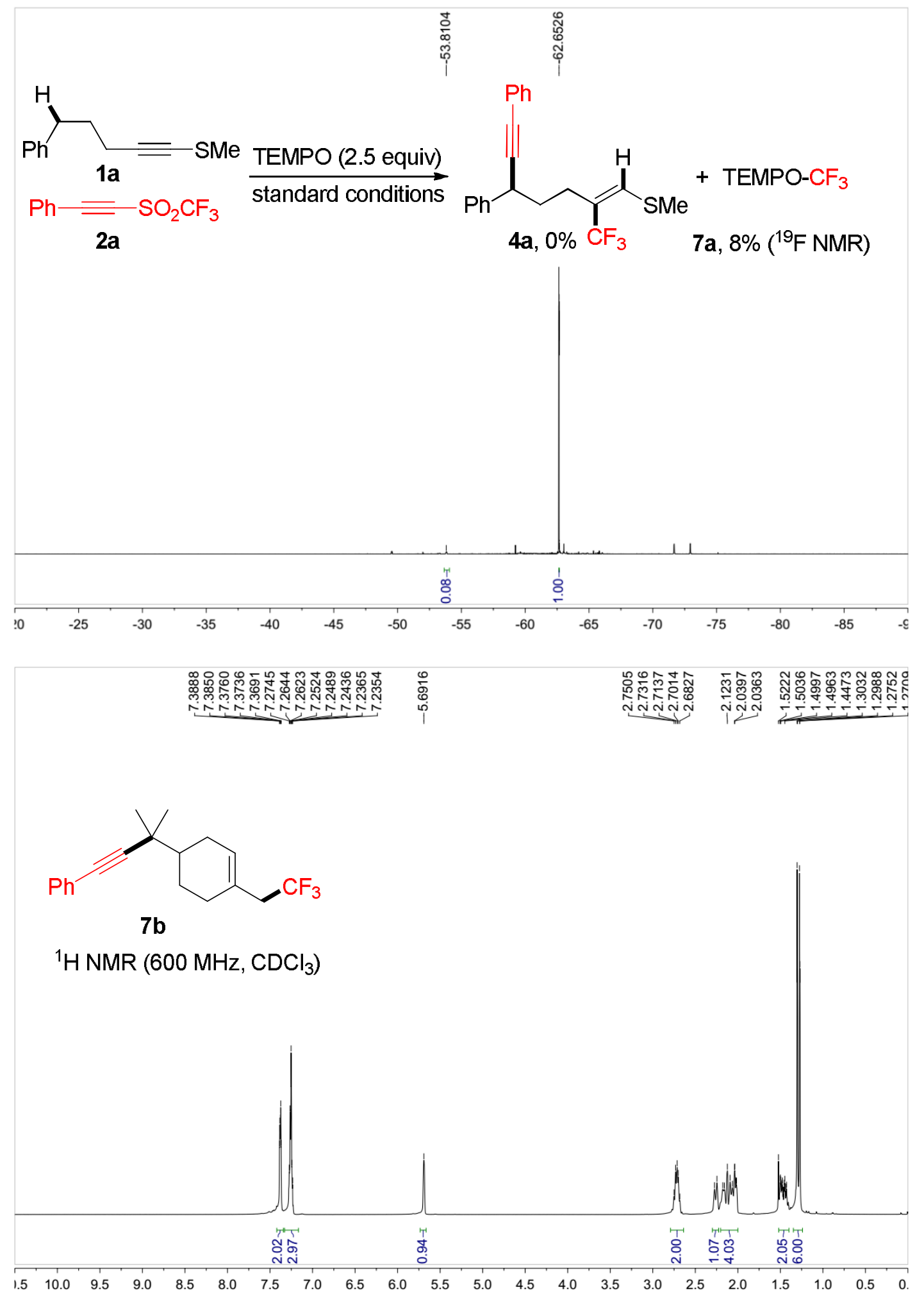




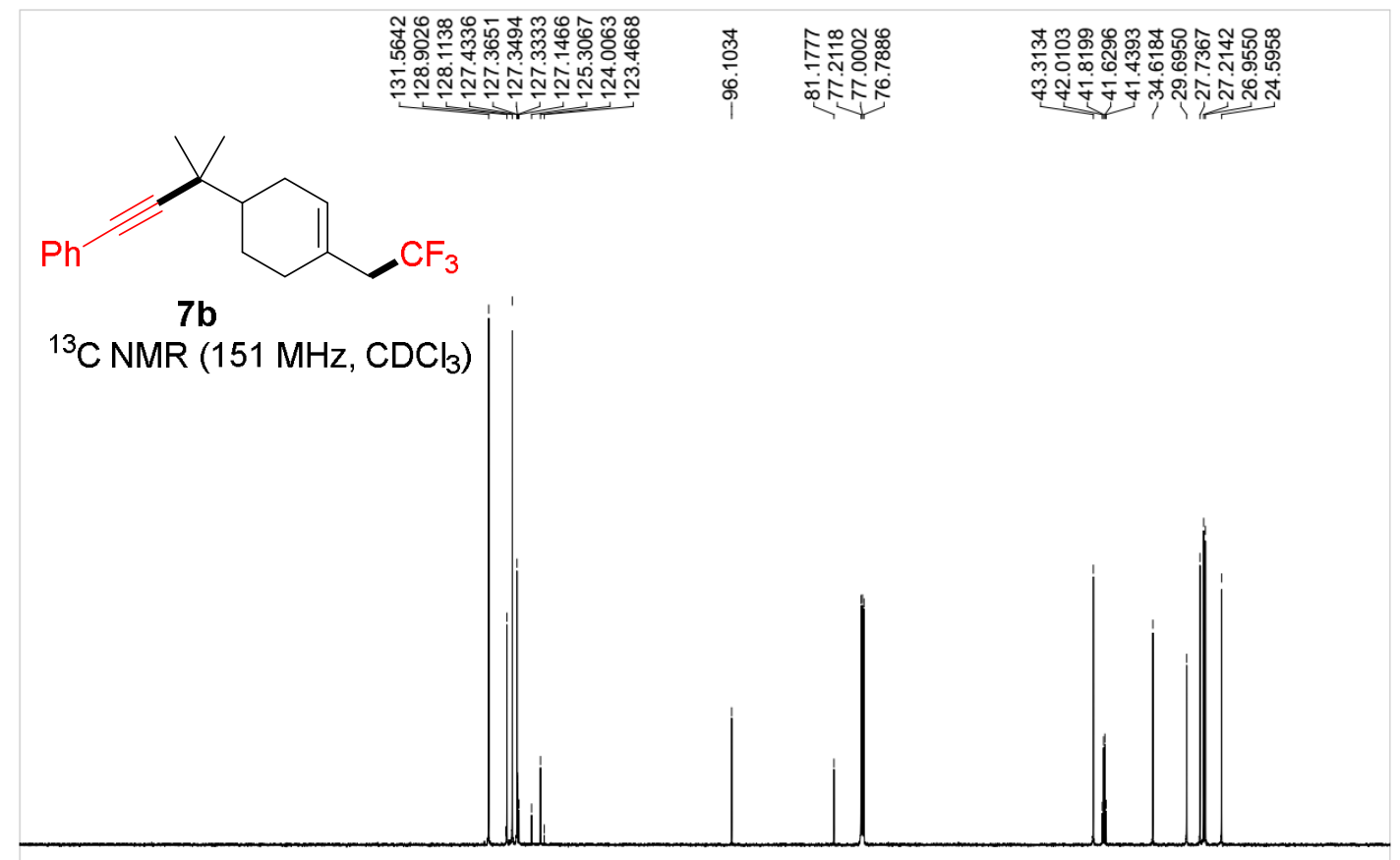

\begin{tabular}{lllllllllllllllllllll}
\hline 0 & 190 & 180 & 170 & 160 & 150 & 140 & 130 & 120 & 110 & 100 & 90 & 80 & 70 & 60 & 50 & 40 & 30 & 20 & 10
\end{tabular}

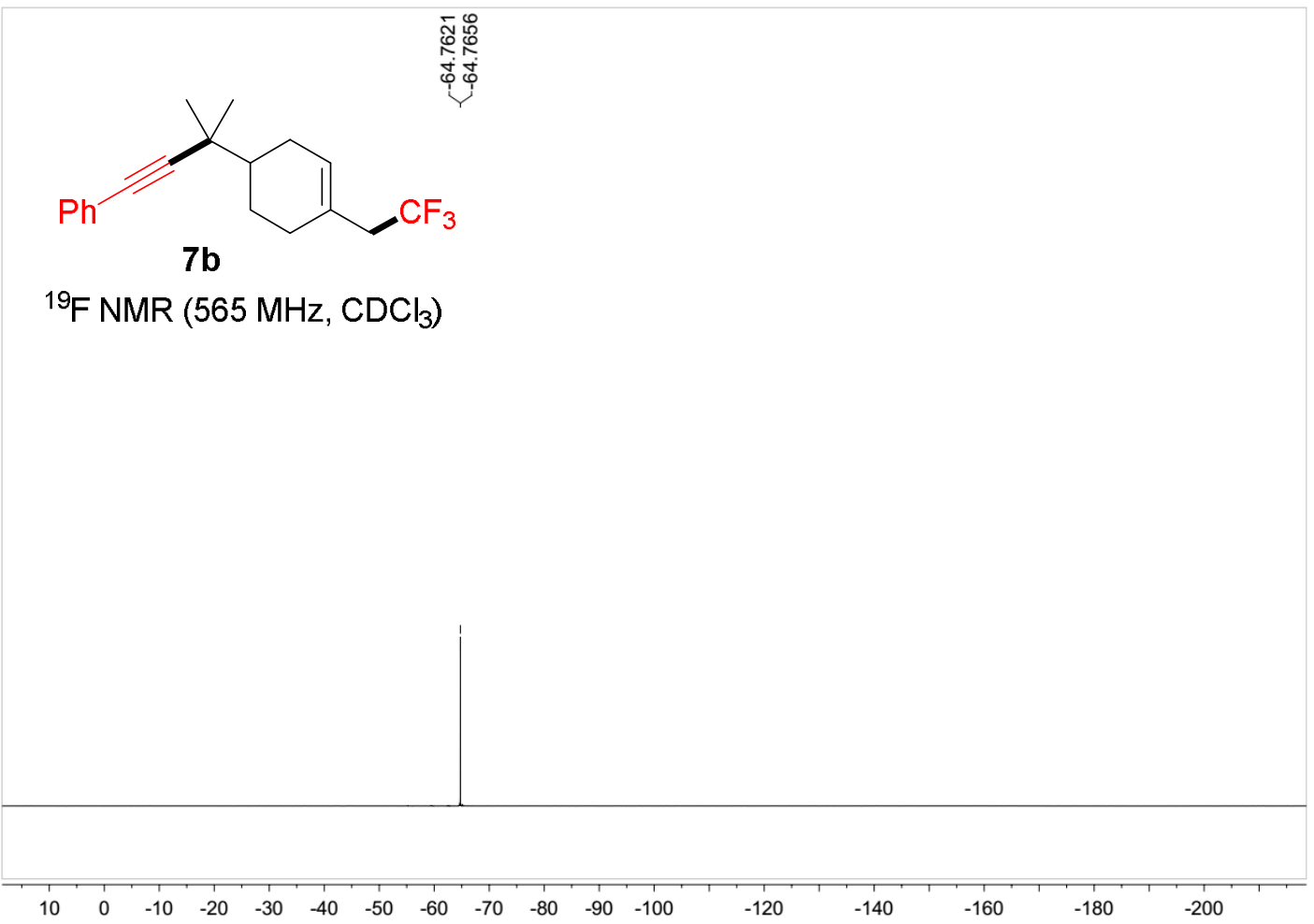




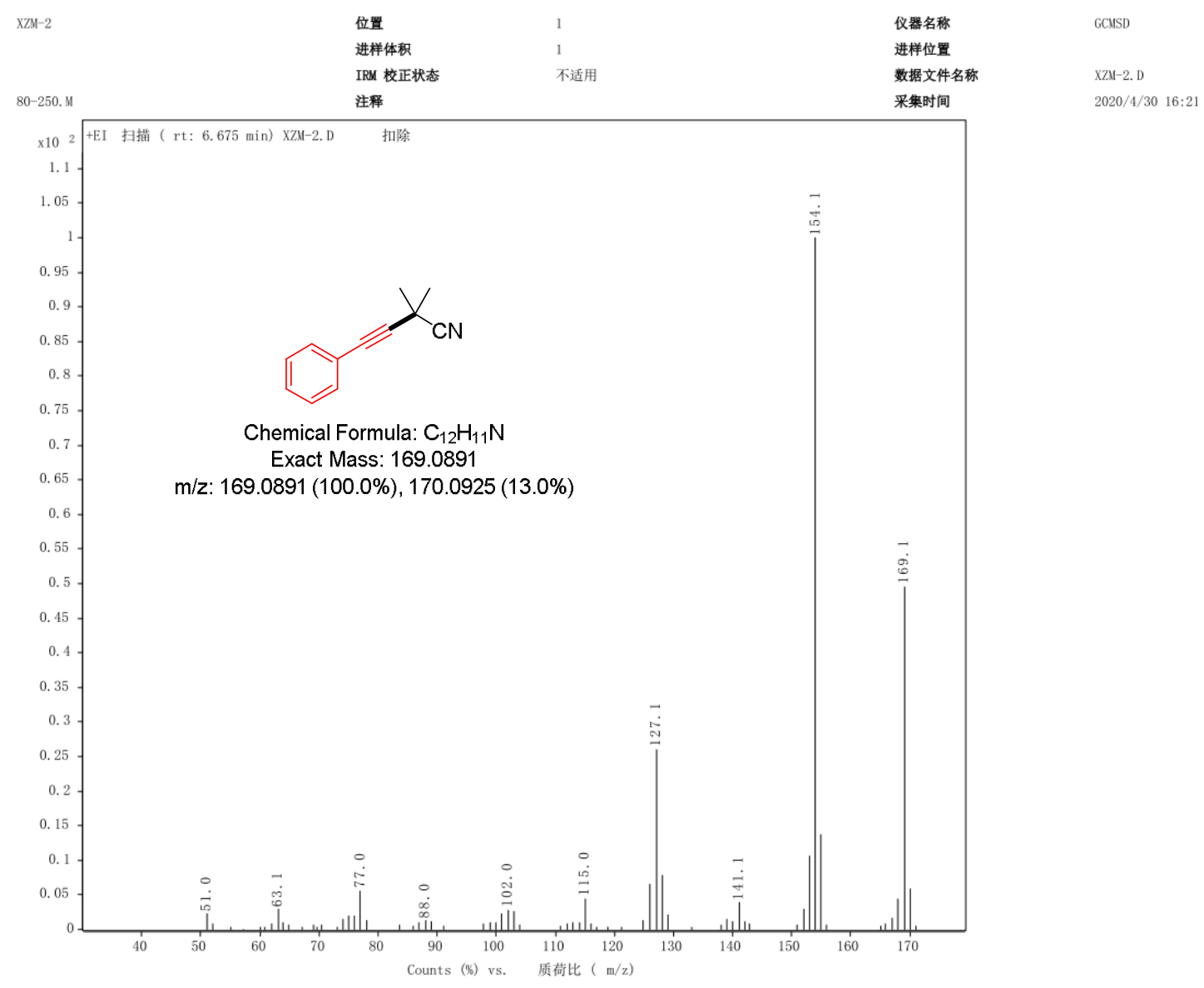

